\title{
New geochemical, U-Pb SIMS geochronology and Lu-Hf isotopic data in zircon from Tandilia basement rocks, Río de la Plata craton, Argentina: Evidence of a sanukitoid precursor for some Paleoproterozoic granitoids
}

\author{
Melisa Angeletti ${ }^{\mathrm{a}, *}$, Martim Chichorro ${ }^{\mathrm{b}}$, Antonio Castro ${ }^{\mathrm{c}}$, María Cristina Frisicale ${ }^{\mathrm{a}}$, Rita Solá ${ }^{\mathrm{d}}$, \\ Luis Vicente Dimieri ${ }^{a}$ \\ ${ }^{a}$ INGEOSUR-CONICET, Universidad Nacional Del Sur, Departamento de Geología, Alem 1253, Cuerpo B', $1^{\circ}$ Piso, Bahía Blanca, 8000 , Argentina \\ ${ }^{\mathrm{b}}$ GeoBioTec, Dept Earth Sciences, Faculty of Science and Technology, New University of Lisbon, Portugal \\ ${ }^{\mathrm{c}}$ Instituto Andaluz de Ciencias de La Tierra (IACT), Consejo Superior de Investigaciones Científicas, Universidad de Granada (CSIC-UGR), Armilla, Granada, 18100, \\ Spain \\ ${ }^{\mathrm{d}}$ Unidade de Geologia, Hidrogeologia e Geologia Costeira, LNEG, Portugal
}

\section{A R T I C L E I N F O}

\section{Keywords:}

Hf $\mathrm{T}_{\mathrm{DM}}$ model Ages

Isotopic signature

Sanukitoids

Modern subduction

Para-autochthonous terranes

Trans-amazonian orogeny

\begin{abstract}
A B S T R A C T
In this paper new geochemical, geochronological and isotopic Lu-Hf data from the Siempre Amigos Hills (SA), western Tandilia, Argentina, is presented. Outcrops in this small area include representative lithologies of the basement Río de la Plata craton (RPC) known as Tandilia Terrane. The new geochemical data characterize a heterogeneous Paleoproterozoic crust with a similar U-Pb SIMS magmatic age $(\sim 2.1 \mathrm{Ga})$ represented by a suite of mafic (amphibolites, charnockitic granulites), intermediate and felsic compositions (diorites, granodiorites and granitic gneisses), as well as intrusive granitic rocks (with Neoproterozoic age of $\sim 652$ Ma in zircon). Geochemical features suggest processes of magmatic differentiation from a mafic igneous protolith associated with the 'Camboriu orogeny' of the Trans-Amazonian-Eburnean cycle. Some amphibolites may represent sanukitoid magmas similar to those formed at the Archean-Proterozoic transition. This paper examines the hypothesis of a sanukitoid precursor for RPC Rhyacian magmatism. The potassic and calc-alkaline character of magmatic series, together with negative $\mathrm{Nb}-\mathrm{Ta}-\mathrm{Ti}$ anomalies and REE patterns, are compatible with classical modern subduction-related magmas. Precise Lu-Hf analyses of zircons collected from a granodiorite and a gneiss indicate a predominantly juvenile source $(+\varepsilon H f)$, with minor crustal recycling $(-\varepsilon H f)$. An inheritance age is recorded in a single Neoarchaean zircon core from a granodiorite (2771 $\pm 15 \mathrm{Ma})$, similar to calculated maximum $\mathrm{Hf} \mathrm{T}_{\mathrm{DM}}(2.8 \mathrm{Ga})$. Considering the new available data from this region of the RPC, a sustainable petrogenetic model is proposed to explain the presence of geochemical and isotopic evidence of sanukitoid magmatism which may have occurred after $2.7 \mathrm{Ga}$, which seems to constitute a magmatic series which is a precursor to the development of the Rhyacian magmatic arc in the Tandilia Terrane. Medium-to high-grade metamorphic conditions probably resulted from Paleoproterozoic subduction followed by the intrusion of huge volumes of Rhyacian calc-alkaline magmatism. This model is compatible with RPC configuration as a collage of terranes, and also consistent with Trans-Amazonian-Eburnean amalgamation of various microcontinents in the formation of Atlantica.
\end{abstract}

\section{Credit author statement}

Melisa Angeletti: Conceptualization, Methodology, Formal analysis, Investigation, writing original draft, review and editing, Visualization. Martim Chichorro: Conceptualization, Formal analysis, Investigation, writing, review and editing, Supervision, Funding acquisition. Antonio
Castro: Methodology, Conceptualization, Validation, review and editing, Supervision, Project administration, Funding acquisition. María Cristina Frisicale: Investigation, Conceptualization, review and editing, Supervision, Project administration, Funding acquisition. Rita Solá; : Formal analysis, Conceptualization, review and editing, Supervision. Luis Vicente Dimieri: Conceptualization, writing, review and editing,

\footnotetext{
* Corresponding author.

E-mail address: melisa.angeletti@uns.edu.ar (M. Angeletti).
} 
Project administration.

\section{Introduction}

The geological history of cratons is of essential importance for the reconstruction of tectonic and magmatic processes occurring in the past that led to the stabilization of present-day continents (Windley, 1995; Frost et al., 1998; Martin et al., 2009). Modern-type subduction is broadly accepted at the end of Archean in association with the generation of magmatic rocks that are diagnostic of changes in the geodynamic process. Among these are characteristic magmatic rock suites such as BADR (basalt, andesite, dacite and rhyolite) and sanukitoids (Lobach-Zhuchenko et al., 2008; Martin et al., 2009; Fowler and Rollinson, 2012). Thus, the identification of subduction-related magmatic rocks, such as andesites and sanukitoids in cratons, is useful for providing a better understanding of tectonic processes at the Archean-Proterozoic transition, when important changes in plate tectonic regimes occurred (Fowler and Rollinson, 2012).

The Rio de la Plata craton (RPC) (Almeida et al., 1973), crops out in both Argentina and Uruguay and is considered one of the main Precambrian shield areas of the South American continent (Fig. 1). It has been studied in recent decades using geochronology, mapping and geochemistry (Almeida et al., 1973; Pankhurst et al., 2003; Rapela et al., 2011; Oyhantçabal et al., 2011, 2018; Cingolani, 2011). The largest outcrop of the RPC in Argentina is found in the Tandilia belt. This comprises an igneous-metamorphic basement known as the Buenos Aires complex (Marchese and Di Paola, 1975), which is composed of tonalitic-to-granitic gneisses, amphibolites, migmatites and granitic rocks, with a few schists, marbles, metavolcanic rocks, and mafic and heterogeneously-deformed ultramafic igneous rocks. This basement is intruded by a swarm of Proterozoic calc-alkaline and tholeiitic dikes (Teixeira et al., 2002) and covered by Neoproterozoic to Lower Paleozoic sediments. In spite of its relevance in the context of the RPC, the
Tandilia belt has received little attention, in particular regarding the origin of magmatic rocks. Their relationship with crustal-making processes and their role in the onset of subduction at the end of Archean may have followed the same process as their Caledonian granitoid precursors (Fowler and Rollinson, 2012). In this paper, we present bulk-rock geochemical data, zircon Lu-Hf isotopic ratios and zircon $\mathrm{U}-\mathrm{Pb}$ age determinations from the Siempre Amigos Hills (SA) in western Tandilia, with the aim of designing an updated tectonic model associated with precursor magmatism in terms of the geodynamic regime from Archean to Proterozoic. Although no larger than $2 \mathrm{~km}^{2}$, many of the lithologies, including granite, amphibolite and gneisses, that characterize the Buenos Aires complex are exposed in this region (Fig. 2). Comparisons with Archean and modern tectonic models and the proposal for consistent petrogenetic evolution are discussed in this paper. Our data support the hypothesis of important Paleoproterozoic crustal growth events (Taylor and McLennan, 1995; Hawkesworth and Kemp, 2006; Dhuime et al., 2012) and provide evidence for the sustainable petrogenetic model of the RPC "terrane collage" for explaining the geochemical and isotopic clues regarding sanukitoid magmatism occurring after $2.7 \mathrm{Ga}$, which seems to constitute a magmatic series precursor to the development of the Rhyacian magmatic arc in the Tandilia Terrane in the context of Trans-Amazonian orogeny. Even though some authors consider the term Trans-Amazonian obsolete, as it had been used indiscriminately to cover all different Paleoproterozoic events of the Siderian, Rhyacian, Orosirian and Statherian periods (e.g. Brito Neves, 2011), it is still used in this paper as the cycle that includes the two main RPC basement-forming orogenies (e.g. Hartmann et al., 2002), which will be explained later.

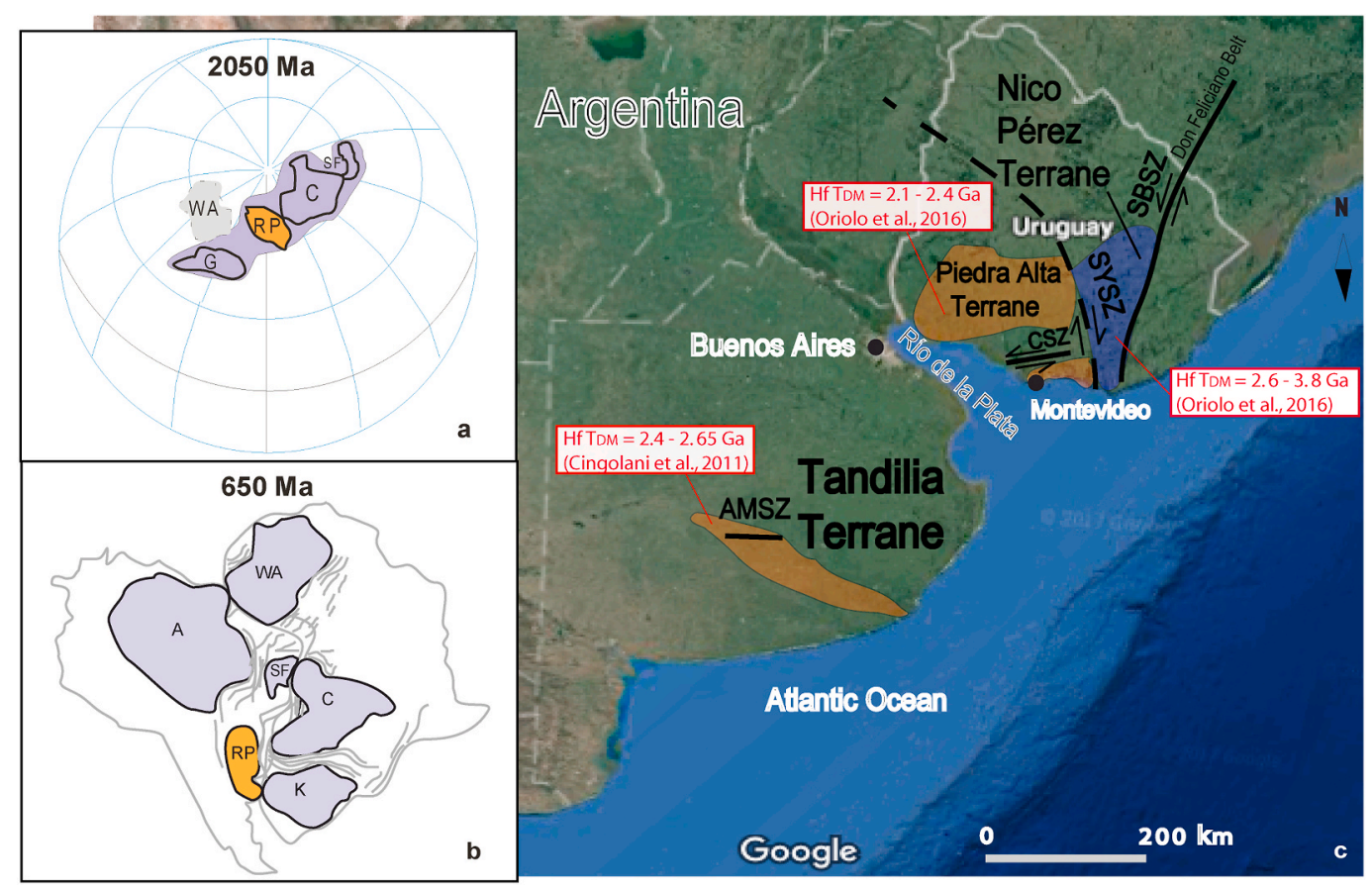

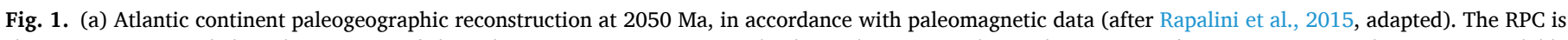

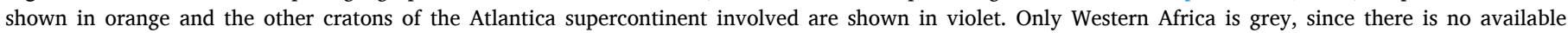

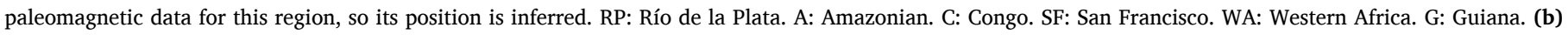

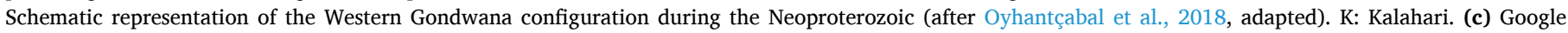

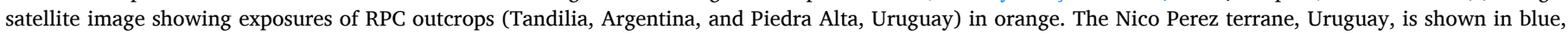

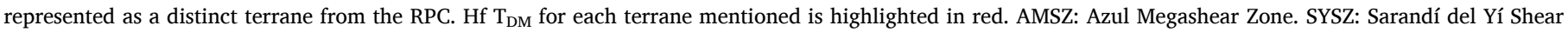
Zone. SBSZ: Sierra Ballena Shear Zone. CSZ: Colonia Shear Zone. 


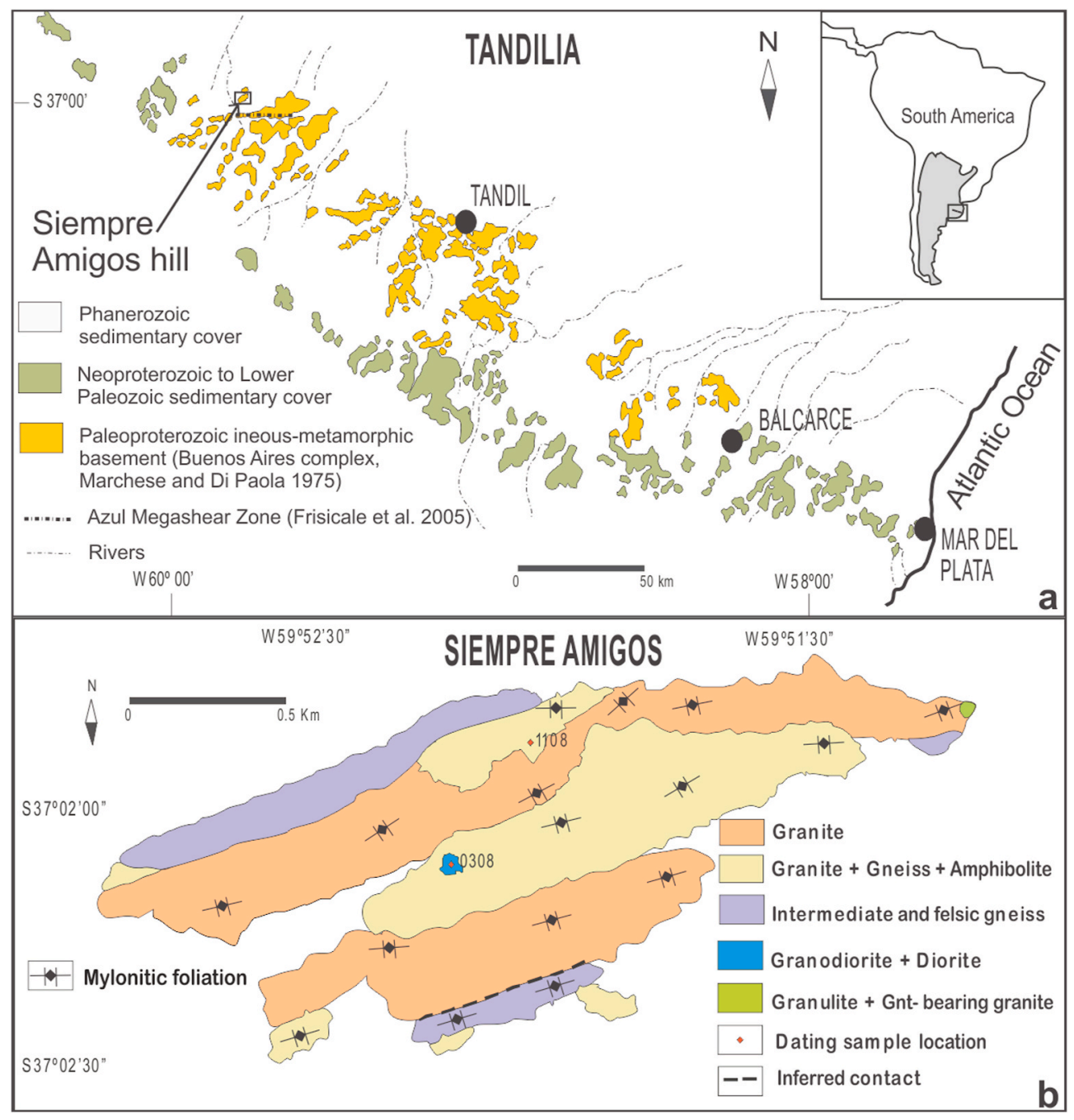

Fig. 2. (a) Geological map of the Tandilia belt, after Iacumin et al. (2001), adapted. (b) Siempre Amigos Hill geological map, scale 1: 12,500. With exception of Granite unit, all lithologies are Paleoproterozoic. Sample locations for Lu-Hf isotopes analyzed only.

\section{Geological background}

\subsection{The RPC and the Paleoproterozoic Atlantica continent}

Rogers (1996) proposed a Paleoproterozoic continent called Atlantica, first defined by Ledru et al. (1994), comprising the cratons of Río de la Plata, Guiana (Proto-Amazonia), Congo, São Francisco and West Africa, which was recently supported and modified by Rapalini et al. (2015) based on new paleomagnetic data (Fig. 1a). Rogers and Santosh (2002) made an initial attempt at global reconstruction for Paleo-Mesoproterozoic times, in which Atlantica, together with Ur (Kalahari, India, West and East of Australia, East Antarctica and Zimbabwe, according to Rogers, 1996) and Nena (Baltica, Siberia, Laurentia and Antarctica, according to Rogers, 1996, also termed Nuna by Hoffman, 1997), were consolidated at $1.8 \mathrm{Ga}$ in a single supercontinent called Columbia (Zhao et al., 2002). Some of the authors who contributed to these paleocontinental reconstructions, such as Tassinari and Macambira (1999); Hartmann (2002); Delor et al. (2003); Pacheco Neves (2003); Barbosa and Sabaté (2004); Oyhantçabal et al. (2011); Rapalini et al. (2015) based their interpretations on similarities in the Paleoproterozoic geological evolution of cratons, supported by geochronological, geochemical and geophysical studies. Rapalini et al. (2015) listed the following features shared by RPC, Congo and São Francisco, West Africa and Amazonia, which provide support for the existence of Atlantica (Fig. 1a and b): (1) High-grade metamorphic terrains of
Paleoproterozoic age (2.3-2.1 Ga), composed of tonalite-trondhjemite-granodiorite (TTG) or "grey gneisses", with juvenile igneous source related to subduction; (2) Supracrustal belts between metamorphic terrains, composed of metasedimentary and metavolcanic rocks in greenschist to amphibolite facies; (3) An episode of major crust formation during the mid-Paleoproterozoic; (4) The Congo-São Francisco, Guiana and West African cratons contain Archean cores, suggesting reworking of an older crust, which is however unlike the RPC, characterized by Neoarchaean $\mathrm{Hf}_{\mathrm{DM}}$ ages. An exception to this is the Nico Perez terrane (NPT) of Uruguay (Fig. 1c), whose assumed linkage to the RPC has been questioned (Oyhantçabal et al., 2011; Oriolo et al., 2016). In this context, Paleoproterozoic orogenic belts between Archean cores characterize the Trans-Amazonian cycle (Almeida et al., 1973). This orogeny caused the amalgamation of Archean (Congo-São Francisco, Guiana and West African) and Early Paleoproterozoic (RPC) microcontinents, leading to the formation of the Atlantica supercontinent (Fig. 1a).

\subsection{Trans-Amazonian cycle: RPC assembly and related terranes}

The Trans-Amazonian cycle includes two main orogenies: 'Encantadas' (Neoarchaean-Siderian: 2.25-2.12 Ga) and 'Camboriu' (Early-Late Rhyacian: 2.10-2.08 Ga) (Hartmann et al., 2002; Cingolani, 2011). They correlate with the Birimian and Eburnean orogenies of Africa (Ledru et al., 1994), respectively. Although the above-mentioned division of the 
Trans-Amazonian cycle was initially proposed for the entire Brazilian Shield (Hartmann 2002), it is almost only used in the context of southern South America. 'Encantadas' accretionary orogeny consists of a subduction event with apparent juvenile arc products. These "juvenile" rocks are considered as having formed as the result of the remelting of an oceanic crust at the convergent margin of a continent (Hartmann et al., 2002). 'Camboriu' is a continent-continent collisional orogeny, manifested as thick mylonitic belts, the presence of ocean-floor rocks and the thickening of the crust. 'Camboriu' orogeny presents large volumes of K-rich granites and volcanic rocks regarded as resulting from the remelting of an older tonalite-rich crust (Hartmann et al., 2002). Thus, different "terrane collages" were proposed, and combined with petrological, geochemical and isotopic evidence to suggest models that explain the tectonic evolution during the Trans-Amazonian cycle. In the context of the RPC, the initial model proposes a magmatic arc-related in Tandilia (Teruggi et al., 1988) with the later obduction of low-grade oceanic metamorphic rocks with island arc-related geochemical affinities termed the El Cortijo formation, which is located between the Buenos Aires and Tandilia continental blocks (Ramos, 1999). The geochemical characterization of the Tandilia basement rocks is coherent with a calc-alkaline signature (Dalla Salda et al., 2006; Pankhurst et al., 2003 and Santos et al., 2017) compatible with intra-oceanic subduction systems or a primitive continental arc setting (Rapela et al., 2007). The latter involves the partial melting of a simple mafic (tholeiitic) source or mixing process between tholeiitic magma and uniformly evolved crust. Isotopic data related to such a magma source produced a range of $\mathrm{T}_{\mathrm{DM}}$ model ages from 2.7 to $2.4 \mathrm{Ga}$ (Hartmann et al., 2002; Pankhurst et al., 2003; Cingolani, 2011, and Santos et al., 2017). The early subduction model was recently modified based on new aeromagnetic data, involving a third terrane, termed Balcarce, to the south of the El Cortijo island arc, and a second suture zone north of El Cortijo, between the Buenos Aires and Tandilia terranes (Chernicoff et al., 2014). Another proposal produced in accordance with new geochronology and $\mathrm{Nd}$ isotope data regards the RPC outcrop in Uruguay, called Piedra Alta terrane (Fig. 1c), which was rapidly formed from a juvenile source (Pamoukaghlian et al., 2017) in contrast with the most protracted history (Cingolani, 2011) in Tandilia. The definition of the Colonia Shear Zone (CSZ in Fig. 1c, Ribot et al., 2005; Bossi and Cingolani, 2009; Gianotti, 2009), as a suture between the two terranes, is consistent with the latter configuration. Other shear zones may also have been involved in the same suture system, such as the Mosquitos shear zone (Oyhantçabal et al., 2006; Sánchez Bettucci et al., 2010).

\subsection{Local geology}

The Siempre Amigos (SA) Hills (Fig. 2a), is located $3 \mathrm{~km}$ north of the Azul Megashear Zone (AMSZ in Figs. 1c and 2a), an east-west subvertical shear zone whose basement is deformed to mylonites and ultramylonites (Frisicale et al., 2001). The general structural and lithological trend is sub-parallel to regional mylonitic foliation, which represents a heterogeneous ductile deformation zone, estimated as having occurred at medium metamorphic grade $\left(400-600{ }^{\circ} \mathrm{C}\right)$, based on data from microdeformation mechanisms in minerals (Angeletti et al., 2016b). The Siempre Amigos region represents a NE-SW-trending belt characterized by (1) elongated, decametric-scale strips of granites (Figs. 2b and 3a) described as the dominant lithology in the region (González Bonorino et al., 1956), (2) bands of intermediate and felsic gneisses and (3) sectors dominated by a heterogeneous mixture of granites, gneisses and amphibolites (Angeletti et al., 2016b) (Figs. 2b and 3b). An aspect to highlight is the appearance of younger Neoproterozoic ( $\sim 652 \mathrm{Ma}) \mathrm{U} / \mathrm{Pb}$ SHRIMP ages in some zircons from SA granite (Angeletti et al., 2014), also identified in granulite zircons. These younger zircon ages are reinterpreted as magmatic, possibly evidencing partial melting in the mylonitic SA granite and this will be developed in a future publication. Contact relationships between the SA rocks analyzed will be detailed in Section 4.1.

\section{Sampling and methodology}

All the lithologies (granitic, gneissic and amphibolitic) present in this heterogenous sector were characterized for whole-rock geochemistry, following field and petrographic characterizations. In the present study, in situ zircon $\mathrm{U}-\mathrm{Pb}$ and $\mathrm{Hf}$ isotopic analyses were obtained from a gneiss of intermediate composition (sample 1008), and a granodiorite (sample 0308).

\subsection{Whole-rock geochemistry}

A total set of 42 samples representative of all lithologies was selected, following petrographic study, for major and trace element analysis. Firstly, 12 granite samples were analyzed at Activation Laboratories Ltd. (ACTLABS), Ontario, Canada. The chemical composition of the samples was measured by means of fusion Inductively Coupled Plasma-Mass Spectrometry (ICP-MS). The rest of the samples of the other lithologies were analyzed at the laboratories of the University of Granada, Spain. Major elements were analyzed by means of X-ray fluorescence (XRF) and trace elements were analyzed using ICP-MS techniques. A compilation of the available geochemical bulk rock of the Tandilia basement was also carried out in order to devise a comparative framework at the regional level. Regarding petrographic analysis, modal proportions of rocks were calculated using the Rock. AR program (Larrea et al., 2014).
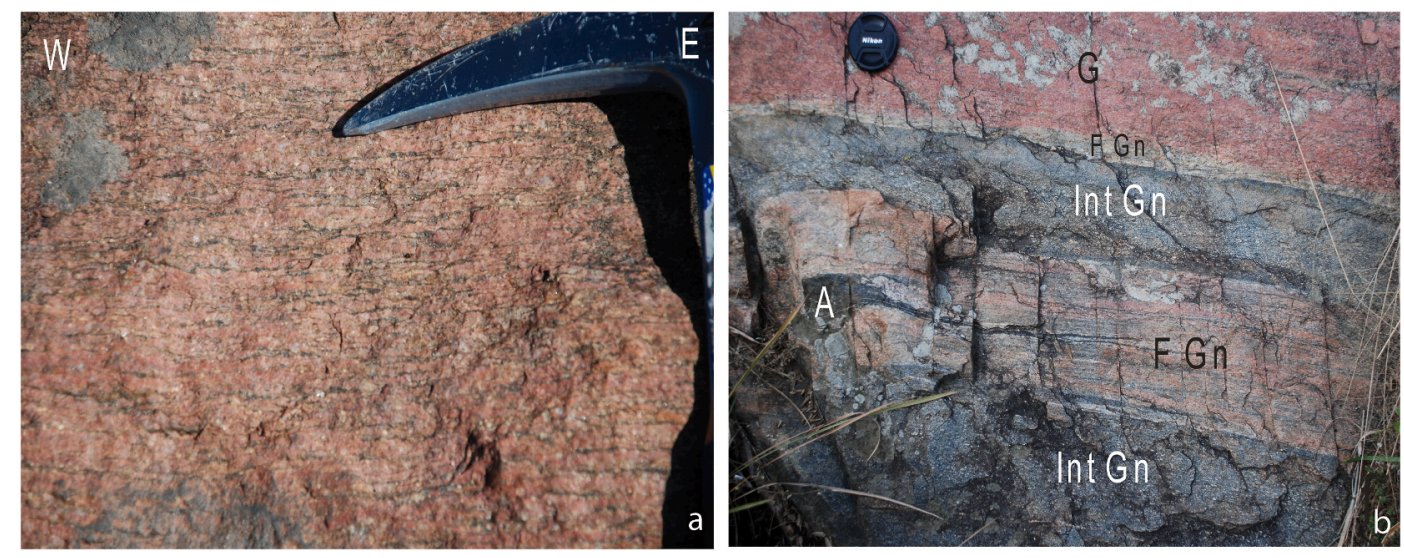

Fig. 3. Field photographs illustrating representative mesoscopic features. (a) Reddish striped gneiss granite, with Qz ribbons indicating mylonitic foliation. (b) A typical outcrop showing intercalation between reddish granite (G), gneiss of intermediate (Int Gn) and felsic (F Gn) composition, and amphibolites (A). 


\subsection{Zircon $U-P b$ geochronology}

The two samples were ground in a ring mill to enable them to pass through a \# 50 disposable nylon sieve. Fine particles were decanted with water. The magnetically susceptible minerals were separated using a hand magnet, and then the zircons were separated using a binocular lens. Zircon grains were handpicked, being selected according to their shape and color, mounted in epoxy together with reference standards, SL-13 (Sri Lanka) as concentration standard and Temora (Australia) as age standard, and polished.

Zircons were imaged by cathodoluminescence (CL) for the selection of spot analyses. After thorough cleaning, mounts were coated with gold (80- $\mu \mathrm{m}$ thick). U-Th-Pb isotopes were analyzed using a SHRIMP IIe/mc instrument at IBERSIMS laboratories, University of Granada, Spain. All calibration procedures were carried out on the standards included on the same mount. Data reduction was performed using SHRIMPTOOLS software (available at www.ugr.es/ fbea). The software is written in STATA commercial package, using powerful algorithms for robust regression, outlier detection and time-series analysis. The result for each isotope was calculated as the value at the mid-point of analyses resulting from the robust regression line. Error ( $95 \%$ confidence level) was calculated as the standard error of linear prediction at the mid-point of analysis. ${ }^{206} \mathrm{~Pb} /{ }^{238} \mathrm{U}$ was calculated from measured ${ }^{206} \mathrm{~Pb}^{+} /{ }^{238} \mathrm{U}^{+}$and $\mathrm{UO}^{+} / \mathrm{U}^{+}$, following the method described by Williams (1998). For high-U zircons (U N $2500 \mathrm{ppm}),{ }^{206} \mathrm{~Pb} /{ }^{238} \mathrm{U}$ was also corrected using the algorithm of Williams and Hergt (2000). Statistical data processing, the calculation of concordia ages and the plotting of concordia diagrams were performed using ISOPLOT software (Ludwig, 2003).

\subsection{Hf isotope data in zircon}

In accordance with their most concordant $\mathrm{U}-\mathrm{Pb}$ ages, zircons of the granodiorite and gneiss were selected for Hafnium isotope measurements at the Geochronology and Isotope Geochemistry-SGIker facility of the University of the Basque Country UPV/EHU, Spain, using a Neptune MC-ICP-MS (Thermo Fisher Scientific) coupled to an UP-213 (New Wave) laser system with a SuperCell laser ablation cell. The zircons were previously placed in a polished-resin test tube (25 mm diameter), carried out at the time of analysis using SHRIMP, so no previous treatment was necessary. Sampling points were selected in accordance with their most concordant ages, taking the spot of previous U-Pb SHRIMP analysis in the zircons as a reference. Data were collected in static mode during $60 \mathrm{~s}$ of ablation with a spot size of $30 \mu \mathrm{m}$. Isotopes ${ }^{171} \mathrm{Yb},{ }^{173} \mathrm{Yb}$ and ${ }^{175} \mathrm{Lu}$ were simultaneously monitored during analysis to enable the correction of isobaric interferences between $\mathrm{Lu}$ and $\mathrm{Yb}$ isotopes for mass 176. Isotopes ${ }^{176} \mathrm{Yb}$ and ${ }^{176} \mathrm{Lu}$ were calculated using the value of 0.796179 for ${ }^{176} \mathrm{Yb} /{ }^{173} \mathrm{Yb}$ and 0.02655 for ${ }^{176} \mathrm{Lu} /{ }^{175} \mathrm{Lu}$ (Chu et al., 2002), while taking into account the instrumental mass fractionation of each individual analysis. To ensure correction for instrumental mass bias, $\mathrm{Yb}$ isotope ratios were normalized to 1.132685 for ${ }^{173} \mathrm{Yb} /{ }^{171} \mathrm{Yb}$ (Chu et al., 2002) and normalized to an isotope ratio of 0.7325 for ${ }^{179} \mathrm{Hf} /{ }^{177} \mathrm{Hf}$ using an exponential law. The mass bias behavior of Lu was assumed to follow that of $\mathrm{Yb}$. Data reduction was performed using the Iolite 2.5 software package for deconvolution of time-resolved data (Paton et al., 2011), using GJ_1 zircon as the standard and Plesovice zircon as a secondary standard.

Isotope-measured ratios were copied to an Excel spreadsheet, for calculation of $\varepsilon \mathrm{Hf}$ and $\mathrm{Hf} \mathrm{T}_{\mathrm{DM}}$ model ages. For the $\varepsilon \mathrm{Hf}_{(\mathrm{t})}$ formula, decay constant $\lambda=1.867 * 10-11$, proposed by Söderlund et al. (2004), and ${ }^{176} \mathrm{Lu} /{ }^{177} \mathrm{Hf}=0.0336$ and ${ }^{176} \mathrm{Hf} /{ }^{177} \mathrm{Hf}=0.282785 \mathrm{CHUR}$ values, according to Bouvier et al. (2008), were used. Two-stage depleted-mantle $\mathrm{Hf}$ model ages, $\mathrm{Hf}_{\mathrm{DM}}$ and $\mathrm{T}_{\mathrm{DM}}^{\mathrm{C}}$, were calculated using ${ }^{176} \mathrm{Lu} /{ }^{177} \mathrm{Hf}=$ 0.0384 and ${ }^{176} \mathrm{Hf} /{ }^{177} \mathrm{Hf}=0.28325$ for depleted mantle (Chauvel and Blinchert-Toft, 2001) and $\mathrm{a}^{176} \mathrm{Lu} /{ }^{177} \mathrm{Hf}$ value of 0.0113 for average crust (Taylor and McLennan 1985; Wedepohl 1995).

\section{Results}

\subsection{Field relationships and petrography}

\subsubsection{Amphibolites}

Amphibolites occur as bands interspersed with granite with an average thickness of $10 \mathrm{~cm}$, and an average foliation attitude of $250^{\circ}$ / $80^{\circ}$ (Fig. 3b). They are homogeneous, fine-grained, dark rocks, predominantly composed of Pl (45\%), Hbl (40\%), scarce Qz (10\%) and Bt (5\%), where mineral abbreviations are according to Whitney and Evans (2010). Ap is the main accessory mineral, plus zircon and Ttn. The rocks exhibit granoblastic textures (Fig. 4a). Amp sometimes occurs as porphyroclasts and is anhedral, locally including Qz grains. Pl is present as anhedral crystals, occasionally with small Bt inclusions. Deformation twins in $\mathrm{Pl}$ and undulatory extinction appear as evidence of intracrystalline deformation. Crystals of $\mathrm{Bt}$ and $\mathrm{Hbl}$ are oriented parallel to the main foliation, this orientation being coincident with amphibolite and gneiss layering.

\subsubsection{Granulites}

In the northeastern margin of the area, an enclave of granulite, of possible restitic origin, approximately $10 \mathrm{~m}$ long and $10 \mathrm{~cm}$ wide, was recognized within the garnetiferous granite. This grey, fine-grained, homogeneous granulite is equigranular, displays a granoblastic texture and has diffuse, gradual contact with the granite. The modal composition (Pl: 52\%; Opx: 18\%; Qz 10\%; Bt 10\%; KFs 7\%; Cpx 2\%; Gnt 1\%, Ap, $\mathrm{Ttn}$, Zrn, and opaque minerals in accessory proportions) is compatible with restitic origin. Bt and orthopyroxene crystals are oriented parallel to the mylonitic foliation (Fig. 4b). Garnet contains biotite, $\mathrm{Pl}$ and $\mathrm{Qz}$ inclusions (Fig. 4c). Incipient core and mantle microstructures in Fsp, deformation and curved twins in $\mathrm{Pl}$, slightly bent $\mathrm{Bt}$, fractured pyroxenes, and Qz with undulating extinction, bulging and subgrains development, all provide microstructural evidence of strong mylonitization. Some $\mathrm{Pl}$ and Fsp crystals show sericitic alteration (Fig. 4c). The rock is classified as felsic granulite according to the IUGS classification (Coutinho et al., 2007) due to the presence of less than $30 \%$ mafic minerals.

\subsubsection{Diorites}

There is a small tabular outcrop of dioritic composition, $10 \mathrm{~cm}$ thick, concordant to the granite, and another small outcrop near the central area (Fig. 2b). The rock is dark, coarse-grained, with lithic polycrystalline porphyroclast composed by $\mathrm{Kfs}$ and $\mathrm{Pl}$, probably having a previous granoblastic texture, dispersed in a matrix with Qz-Fsp-Bt composition (Fig. 4d). Rock texture consists of large porphyroclasts in a fine-grained foliate matrix. Porphyroclasts are Pl with deformation twins (48\%), Amp (13\%) and Kfs (1\%). Incipient sericitic alteration is detected at contacts between feldspars (Fig. 4d). Amp appears as large porphyroclasts, including Qz (30\%) and biotite (8\%). Qz crystals display the development of incipient ribbons. As accessory minerals, Zrn, Ap, $\mathrm{Ttn}, \mathrm{Aln}$ and opaque minerals with a square morphology were observed.

\subsubsection{Gneisses}

Gneisses of intermediate composition are foliated, grey, mediumgrained banded rocks, interleaved with felsic gneiss and amphibolites. They are found in large massive outcrops, parallel to granite foliation (Fig. 3b). Banding is defined by mafic minerals: $\mathrm{Hbl}(10 \%)$ as porphyroclasts, and Bt (8\%), grouped and oriented in accordance with main foliation. Between these rock bands, felsic minerals such as Kfs and Mc (25\%), Pl (27\%) and Qz (30\%) are part of a fine-grained granoblastic matrix (Fig. 4e). The most salient deformation feature is the curved twins observed in $\mathrm{Pl}$ crystals. In some sectors, minor porphyric orthopyroxene is observed, slightly elongated and curved, associated with Amp and Bt by retrogradation. Aln, Zrn and Ap are present as accessory minerals. Chlorite replaces Bt.

Felsic gneisses, occurring as $10 \mathrm{~cm}$ wide bands interleaved with intermediate gneiss (Fig. 3b), have similar textures to the grey gneisses, 

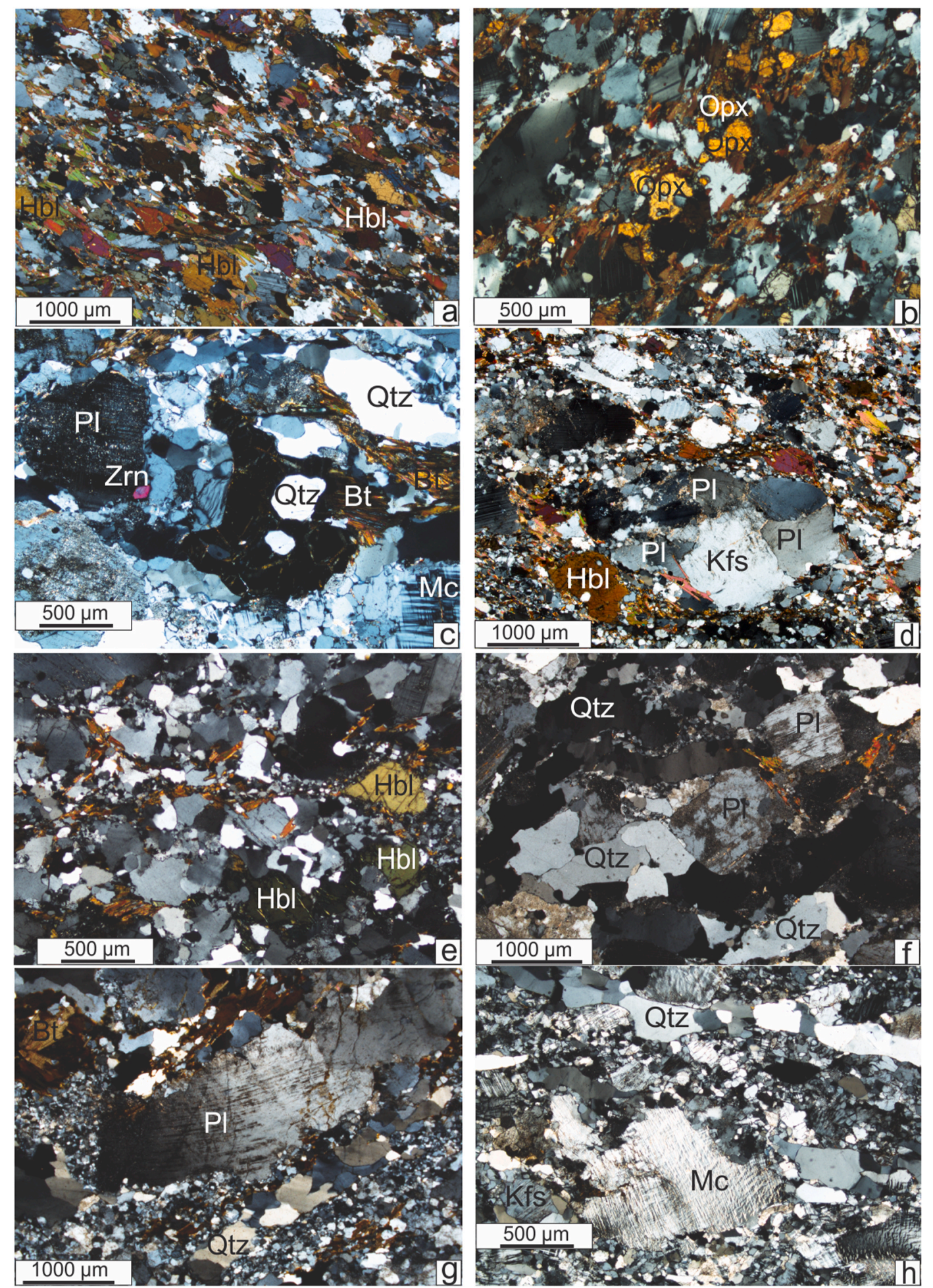

Fig. 4. Photomicrographs of lithologies analyzed (cross-polarized light). (a) Amphibolite with granoblastic texture and slightly larger porphyroblasts of Hbl and Bt oriented with main foliation. (b) Felsic granulite showing granoblastic texture, with orthopyroxene crystals oriented in accordance with mylonitic foliation. (c) Garnet porphyroblast in granite that hosts a granulite enclave. (d) Diorite with felsic lenses and large Fsp, $\mathrm{Pl}$ and Hbl porphyroclasts dispersed in a fine-grained foliate matrix of dioritic composition. Rock texture consists of large porphyroclasts in matrix. (e) Gneiss of intermedian composition, exposed banding of rock defined by mafic minerals oriented with foliation: Hbl porphyroclasts and Bt. Felsic domains with Qz and Fsp show a fine granoblastic arrangement. (f) Felsic gneiss, with Pl porphyroclast with sericitic alteration, and slightly developed Qz ribbons. (g) Pl porphyroclasts in granodiorite, showing curved deformation twins and undulose extinction. (h) Granitic ribbon mylonite, with developed ribbons of Qz and recrystallized matrix with polygonal texture along with other felsic minerals, between porphyroclasts of Fsp and Mc. Abbreviations: $\mathrm{Hbl}=$ hornblende, $\mathrm{Opx}=$ orthopyroxene, $\mathrm{Gt}=$ garnet, $\mathrm{Qtz}=$ quartz, $\mathrm{Bt}=$ biotite, $\mathrm{Pl}=\mathrm{plagioclase}, \mathrm{Zrn}=\mathrm{zircon}, \mathrm{Mc}=$ microcline, Kfs = K-feldspar. 
with poorly-developed ribbon Qz and locally polygonal texture in the felsic matrix. Modal composition is: Qz (35\%), Pl (19\%), Kfs and Mc (45\%), as well as scarce Bt (2\%) (Fig. 4f). Accessory phases, such as Aln, Ap and Zrn, were observed. Generally, in the gneiss, most Fsp porphyroclasts show abundant sericitic alteration.

\subsubsection{Granodiorites}

Granodiorites only appear in a small central outcrop, occurring near diorite, with no clear relationships with the other rocks. In macroscopic terms, a salient feature is $\mathrm{Pl}$ and perthitic Fsp porphyroclasts with tabular shapes, with a length of up to $3 \mathrm{~cm}$. Pl (51\%) presents curved deformation twins and undulose extinction. Some Kfs porphyroclasts (21\%) have myrmekitic contacts and flame perthites. Bt (10\%) is the only mafic phase, being locally abundant and oriented within the main foliation and localized in specific areas in association with magnetite and ilmenite. Qz (18\%) is present in poorly-developed ribbons, with recrystallized Kfs and $\mathrm{Pl}$ in a mosaic matrix (Fig. 4g). Some Fsp crystals present sericitic alteration. Besides opaque minerals, large Aln crystals, abundant large Zrn and Ap are present as accessory minerals.

\subsubsection{Granites}

High-grade mylonitic deformation led to the development of granitic-striped gneisses (Passchier and Trouw, 2005), with Qz ribbons paralleling the $\mathrm{N} 250^{\circ}$ foliation, alternated with a feldspathic matrix (Fig. 3a). The most common granite modal composition is: Qz (45\%), Kfs (Mc and perthitic Fsp, 42\%), Pl (10\%) and Bt (3\%). Qz is the main constituent of ribbons and a recrystallized polygonal-texture felsic matrix. Some porphyroclasts of Fsp and smaller amounts of $\mathrm{Pl}$ are observed in ribbon mylonites in a very low proportion in the matrix. Other characteristic deformation features are undulose extinction, subgrains in $\mathrm{Qz}$ and incipient core-mantle texture around Fsp porphyroclasts (Fig. 4h). Accessory minerals are Aln, Zrn and opaque minerals. Sericitic alteration is extremely intense in some Kfs and Pl crystals, accompanied by some secondary muscovite crystals.

\subsection{Whole rock geochemistry}

Representative whole rock analyses are listed in Table 1. The main geochemical features are compared with a compilation of similar chemical data published to date for the Paleoproterozoic basement of Tandilia (Di Paola and Espiasse, 1986; Dalla Salda et al., 1992; Di Paola, 1995; Iacumin et al., 2001; Frisicale et al., 2005; Martínez and Dristas, 2007; Jorgensen et al., 2008; Frisicale et al., 2012; Massonne et al., 2012).

Harker diagrams (Fig. 5) show a trend consistent with differentiation processes, with regular correlations, suggesting that the magmatic signature is preserved despite intense deformation. Values for element abundances decrease for the most differentiated terms, with the exception of $\mathrm{K}_{2} \mathrm{O}$ and $\mathrm{Na}_{2} \mathrm{O}$, whose contents increase with differentiation. The least differentiated samples correspond to amphibolites, which are characterized by a content of $54 \% \mathrm{SiO}_{2}$ and high percentages of $\mathrm{MgO}$ (3.67-4.65\%). Amphibolite and diorite samples are rich in $\mathrm{K}_{2} \mathrm{O}$. Granites have a high silica content ( $>75 \%$ ). Gneisses, diorites, granodiorites and granulites range from mafic to felsic. Regarding $\mathrm{TiO}_{2}$ behavior as compared with that of $\mathrm{SiO}_{2}$, in addition to decreasing with differentiation, content for the least differentiated terms is around $1 \mathrm{wt} \%$.

In the diagram, for total alkalis versus silica or TAS (Le Bas et al., 1986) (Fig. 6a), all rocks are sub-alkaline or tholeiitic, despite having high potassium values. Amphibolites are chemically classified as basaltic trachyandesites and the gneisses of intermediate composition as andesites and dacites.

The $\mathrm{Zr} / \mathrm{TiO}_{2}$ versus $\mathrm{Nb} / \mathrm{Y}$ rock classification diagram of Pearce (1996) (Fig. 6b) shows amphibolite, diorite, and most intermediate gneiss samples chemically classified as andesite/basaltic andesite. Granulites are of trachyandesite composition. Granodiorites and two intermediate gneiss samples plot as rhyolite/dacite and felsic gneiss between alkali rhyolite and trachyte fields. Data for granitic samples are greatly dispersed, ranging from rhyolite/dacite, alkali rhyolite to trachyte. For most samples, the aluminum-saturation index (ASI) (Frost and Frost, 2008a) (Fig. 6c) is around 1.1, characterizing granites as slightly peraluminous. In the diagram of Frost et al. (2001), most rocks plot in the ferroan field, giving high $\mathrm{Fe}^{*}$ values (Fig. 6d). The Paleoproterozoic rocks of SA have compositional ranges similar to those of Tandilia units available in the literature (Fig. 6).

In the graphic devised by Peccerillo and Taylor (1976) plotting $\mathrm{K}_{2} \mathrm{O}$ versus $\mathrm{SiO} 2$ (Fig. 7a), SA rocks are identified as K-rich calc-alkaline. Another diagram plotting $\mathrm{K}_{2} \mathrm{O} /\left(\mathrm{K}_{2} \mathrm{O}+\mathrm{CaO}\right.$ ) versus $\mathrm{MgO}$ (Fig. $7 \mathrm{~b}$ ) shows the alignment of samples along a possible differentiation line. Some gneisses, granites and granulites provide exceptions, displaying lower values of $\mathrm{K}_{2} \mathrm{O} / \mathrm{K}_{2} \mathrm{O}+\mathrm{CaO}$.

A series of diagrams was selected in order to establish a geochemical comparison between SA rocks and modern adakites. In this paper, adakites are regarded as being analogous to Archean TTG complexes (Martin, 1986, 1999; Martin and Moyen, 2012). The main differences between adakites and calc-alkaline rocks are in the rare earth element patterns and the $\mathrm{Sr} / \mathrm{Y}$ ratio.

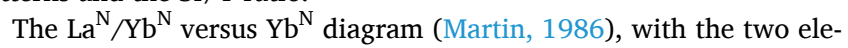
ments normalized to chondrites, leads to the discrimination of Archean adakites and TTGs from post-Archean granitoids (Fig. 7c). Amphibolites and intermediate gneisses are much depleted in both elements, together with granites and SA felsic gneisses, displaying $\mathrm{La}^{\mathrm{N}} / \mathrm{Yb}^{\mathrm{N}}$ ratios from 0.1 to 25 similar that of post- $2.5 \mathrm{Ga}$ granitoids. There is a general trend for all rocks taken together, showing a curve with $\mathrm{La}^{\mathrm{N}} / \mathrm{Yb}^{\mathrm{N}}$ increasing as $\mathrm{Yb}^{\mathrm{N}}$ decreases, which may mean that $\mathrm{Yb}$ is fractionated in a garnet- and Hbl-rich residue.

The $\mathrm{Sr} / \mathrm{Y}$ versus $\mathrm{Y}$ diagram (Fig. 7d) shows discrimination fields according to Drummond and Defant (1990) between adakites and normal calc-alkaline rocks. SA rocks plot like normal calc-alkaline rocks, with $\mathrm{Sr} / \mathrm{Y}$ ratios ranging from 20 to 52 . Most of the granites and felsic gneisses are depleted in $\mathrm{Sr} / \mathrm{Y}$, displaying below-normal values for adakite rocks, which is coherent with the normal-calc-alkaline characterization of all SA rocks, in accordance with Fig. $7 \mathrm{~d}$. The red arrow (Fig. 7d) shows the $\mathrm{Y}$ increment from Grt-bearing granulites to diorites and granites and a very slight decrease in the Sr/Y ratio. The Tandilia database provided similar results for SA rocks. There is not even a single sample in the adakite field, except four samples from Tandilia: diorite and gabro from Tandil (Iacumin et al., 2001) and two granulites from Cerro Negro (Frisicale et al., 2012). Whatever the case, the trend for Tandilia geochemical derivation is similar to that of SA rocks, for which the interpretation of normal-calc-alkaline is extended to the rest of the Tandilia basement rocks.

Fig. 8, a diagram modified after Stevenson et al. (1999), shows the content of $\mathrm{Mg} \#$ versus $\mathrm{Ni}$ of the SA rocks, in agreement with that which is expected for sanukitoids (op. cit.), and poorer in $\mathrm{Mg \#}$ than adakites.

Chondrite-normalized REE diagrams are shown in Fig. 9. REE patterns for amphibolites, granulites and intermediate gneisses are very similar, showing affinities with classical andesite patterns. Amphibolites samples seem to be the most coherent group as this is the least modified by deformation and metamorphism. They show a typical pattern, which is HREE-depleted and concave, indicating magmatic equilibrium with a mafic residue dominated by Cpx and Amp. Granulites and intermediate gneisses inherit the amphibolite pattern, while diorites show more fractionation in terms of REE, with enrichment of LREE and depletion of HREE. Intermediate and felsic gneisses and granodiorite show marked negative Eu anomalies, attributed to plagioclase fractionation. Granitic samples display variable REE patterns. Only a few granite samples show depletion of HREE, with negative Eu anomalies (see orange broken line in Fig. 9). Garnetiferous granite REE is highlighted (see continuous red line in Fig. 9). Incompatible elements normalized to MORB are shown in Fig. 10, with similar patterns in amphibolites and granulites, showing $\mathrm{Nb}$ and $\mathrm{Ti}$ negative anomalies, elements present in rutile, ilmenite and sphene. Intermediate and felsic gneiss, diorite and granite inherit these 
Table 1

Major (wt. \%) and trace element (ppm) concentrations of the Siempre Amigos hill, Tandilia, Argentina.

\begin{tabular}{|c|c|c|c|c|c|c|c|c|c|}
\hline Rock type $^{\mathrm{a}}$ & Amphibolite & Amphibolite & Amphibolite & FCG & FCG & FCG & Diorite & Diorite & Granite \\
\hline Sample & 05081212 & 09081212 & 06061211 & 11081212 & 05010813 & 02020813 & 020801212 & 01081212 & 06081212 \\
\hline Longitude $^{\mathrm{b}}$ & $59^{\circ} 52^{\prime} 2^{\prime \prime}$ & $59^{\circ} 52^{\prime} 3^{\prime \prime}$ & $59^{\circ} 51^{\prime} 51^{\prime \prime}$ & $59^{\circ} 52^{\prime} 3^{\prime \prime}$ & $59^{\circ} 52^{\prime} 4^{\prime \prime}$ & $59^{\circ} 52^{\prime} 32^{\prime \prime}$ & $59^{\circ} 52^{\prime} 12^{\prime \prime}$ & $59^{\circ} 52^{\prime} 29^{\prime \prime}$ & $59^{\circ} 52^{\prime} 4^{\prime \prime}$ \\
\hline Latitude $^{\mathrm{b}}$ & $37^{\circ} 1^{\prime} 56^{\prime \prime}$ & $37^{\circ} 1^{\prime} 54^{\prime \prime}$ & $37^{\circ} 1^{\prime} 59^{\prime \prime}$ & $37^{\circ} 1^{\prime} 54^{\prime \prime}$ & $37^{\circ} 1^{\prime} 54^{\prime \prime}$ & $37^{\circ} 2^{\prime} 5^{\prime \prime}$ & $37^{\circ} 2^{\prime} 7^{\prime \prime}$ & $37^{\circ} 2^{\prime} 21^{\prime \prime}$ & $37^{\circ} 1^{\prime} 55^{\prime \prime}$ \\
\hline $\mathrm{SiO}_{2}$ & 54.11 & 54.21 & 53.84 & 76.33 & 78.90 & 73.83 & 59.95 & 63.17 & 77.49 \\
\hline $\mathrm{TiO}_{2}$ & 0.83 & 0.79 & 0.81 & 0.08 & 0.01 & 0.21 & 1.13 & 0.60 & 0.05 \\
\hline $\mathrm{Al}_{2} \mathrm{O}_{3}$ & 17.75 & 16.63 & 16.30 & 12.99 & 12.75 & 13.74 & 16.57 & 16.21 & 12.53 \\
\hline $\mathrm{FeO}$ & 8.15 & 9.18 & 8.96 & 1.02 & 0.93 & 2.36 & 6.76 & 5.17 & 0.79 \\
\hline $\mathrm{MgO}$ & 3.67 & 4.09 & 4.54 & 0.15 & 0.15 & 0.36 & 1.64 & 2.05 & 0.04 \\
\hline $\mathrm{MnO}$ & 0.21 & 0.28 & 0.19 & 0.02 & 0.03 & 0.02 & 0.11 & 0.11 & 0.01 \\
\hline $\mathrm{CaO}$ & 7.07 & 6.44 & 6.70 & 0.97 & 1.79 & 1.32 & 4.11 & 4.17 & 0.37 \\
\hline $\mathrm{Na}_{2} \mathrm{O}$ & 3.81 & 3.38 & 4.05 & 2.88 & 3.83 & 2.89 & 2.56 & 3.39 & 2.74 \\
\hline $\mathrm{K}_{2} \mathrm{O}$ & 1.70 & 2.13 & 2.01 & 4.54 & 0.75 & 3.88 & 4.77 & 2.88 & 5.19 \\
\hline $\mathrm{P}_{2} \mathrm{O}_{5}$ & 0.37 & 0.28 & 0.32 & 0.05 & 0.02 & 0.08 & 0.53 & 0.23 & 0.03 \\
\hline LOI & 0.62 & 0.75 & 0.90 & 0.66 & 0.39 & 0.63 & 0.43 & 0.64 & 0.51 \\
\hline Total & 98.29 & 98.16 & 98.62 & 99.70 & 100.58 & 101.96 & 106.08 & 104.36 & 99.75 \\
\hline \multicolumn{10}{|c|}{ Trace elements (ppm) } \\
\hline $\mathrm{Li}$ & 42.87 & 78.66 & 0.00 & 10.62 & 8.76 & 47.97 & 20.78 & 59.14 & 6.24 \\
\hline $\mathrm{Be}$ & 5.78 & 2.95 & 4.00 & 2.49 & 2.81 & 2.19 & 2.87 & 2.84 & 0.66 \\
\hline $\mathrm{Sc}$ & 21.77 & 28.74 & 26.00 & 1.29 & 11.67 & 9.09 & 16.29 & 14.53 & 2.20 \\
\hline V & 118.99 & 165.94 & 166.00 & 16.15 & 24.05 & 35.14 & 77.54 & 83.59 & 7.66 \\
\hline $\mathrm{Cr}$ & 132.99 & 180.81 & 0.01 & 316.48 & 392.04 & 463.23 & 295.18 & 354.95 & 150.17 \\
\hline Co & 42.78 & 45.93 & 29.30 & 51.74 & 1.46 & 2.75 & 12.10 & 12.34 & 75.39 \\
\hline $\mathrm{Ni}$ & 17.51 & 13.69 & $<20$ & 10.52 & 11.89 & 16.15 & 16.02 & 20.04 & 5.80 \\
\hline $\mathrm{Cu}$ & 10.73 & 26.48 & 183.00 & 43.10 & 8.17 & 7.95 & 30.77 & 30.62 & 21.58 \\
\hline $\mathrm{Zn}$ & 137.56 & 180.61 & 111.00 & 27.04 & 26.93 & 33.81 & 120.25 & 75.69 & 15.59 \\
\hline $\mathrm{Ga}$ & 22.33 & 22.96 & 18.10 & 21.85 & 21.67 & 19.95 & 26.01 & 20.52 & 22.76 \\
\hline $\mathrm{Rb}$ & 169.16 & 388.26 & 282.50 & 236.01 & 38.13 & 164.50 & 177.16 & 150.99 & 233.47 \\
\hline $\mathrm{Sr}$ & 742.93 & 567.47 & 572.40 & 81.70 & 102.36 & 124.52 & 735.59 & 668.34 & 24.73 \\
\hline $\mathrm{Y}$ & 19.46 & 24.43 & 15.30 & 6.69 & 3.69 & 21.15 & 32.30 & 23.21 & 10.54 \\
\hline $\mathrm{Zr}$ & 89.40 & 111.10 & 111.30 & 112.70 & 60.00 & 189.80 & 1007.50 & 172.40 & 79.70 \\
\hline $\mathrm{Nb}$ & 6.14 & 9.38 & 6.40 & 3.22 & 1.16 & 19.40 & 29.03 & 12.11 & 2.64 \\
\hline Cs & 6.27 & 18.52 & 17.30 & 1.07 & 0.45 & 1.33 & 1.04 & 3.16 & 0.47 \\
\hline $\mathrm{Ba}$ & 764.94 & 858.32 & 1232.00 & 218.28 & 114.36 & 532.98 & 3743.48 & 1257.40 & 66.07 \\
\hline $\mathrm{La}$ & 37.37 & 33.41 & 25.70 & 10.14 & 17.64 & 16.50 & 175.21 & 50.69 & 8.33 \\
\hline $\mathrm{Ce}$ & 77.44 & 67.77 & 54.40 & 17.37 & 28.50 & 37.40 & 352.37 & 101.82 & 12.64 \\
\hline $\operatorname{Pr}$ & 9.70 & 8.32 & 6.71 & 1.83 & 2.75 & 4.74 & 38.88 & 11.50 & 1.36 \\
\hline $\mathrm{Nd}$ & 38.30 & 32.70 & 28.40 & 7.26 & 9.92 & 19.54 & 136.79 & 43.00 & 6.06 \\
\hline $\mathrm{Sm}$ & 6.95 & 6.54 & 5.28 & 1.34 & 1.46 & 5.01 & 19.25 & 7.56 & 1.43 \\
\hline $\mathrm{Eu}$ & 2.01 & 1.67 & 1.37 & 0.31 & 0.22 & 0.53 & 4.08 & 1.52 & 0.14 \\
\hline Gd & 5.06 & 5.21 & 4.30 & 1.33 & 1.00 & 4.62 & 12.70 & 5.58 & 1.50 \\
\hline $\mathrm{Tb}$ & 0.66 & 0.75 & 0.60 & 0.19 & 0.13 & 0.68 & 1.60 & 0.77 & 0.26 \\
\hline Dy & 3.34 & 4.33 & 3.39 & 1.11 & 0.64 & 3.82 & 6.45 & 3.98 & 1.69 \\
\hline Ho & 0.64 & 0.85 & 0.59 & 0.24 & 0.12 & 0.75 & 1.18 & 0.78 & 0.40 \\
\hline $\mathrm{Er}$ & 1.56 & 2.33 & 1.53 & 0.68 & 0.33 & 1.83 & 2.80 & 1.98 & 1.19 \\
\hline $\mathrm{Tm}$ & 0.26 & 0.38 & 0.24 & 0.12 & 0.06 & 0.27 & 0.40 & 0.32 & 0.22 \\
\hline $\mathrm{Yb}$ & 1.59 & 2.44 & 1.54 & 0.70 & 0.45 & 1.36 & 2.23 & 1.90 & 1.37 \\
\hline $\mathrm{Lu}$ & 0.25 & 0.39 & 0.23 & 0.12 & 0.07 & 0.22 & 0.34 & 0.29 & 0.23 \\
\hline $\mathrm{Hf}$ & 1.76 & 2.34 & 2.80 & 5.72 & 2.71 & 6.62 & 10.11 & 4.55 & 3.04 \\
\hline $\mathrm{Ta}$ & 0.49 & 0.89 & 0.30 & 0.64 & 0.09 & 0.62 & 0.90 & 0.89 & 0.59 \\
\hline W & 0.00 & 0.00 & 1.20 & 0.00 & 0.00 & 0.00 & 0.00 & 0.00 & 0.00 \\
\hline $\mathrm{Pb}$ & 16.43 & 11.74 & 2.30 & 42.36 & 21.06 & 34.38 & 21.71 & 20.35 & 37.16 \\
\hline Th & 3.21 & 3.60 & 2.50 & 11.45 & 3.59 & 36.02 & 6.38 & 12.37 & 5.70 \\
\hline U & 1.68 & 1.19 & 0.70 & 3.46 & 0.81 & 4.20 & 1.19 & 3.23 & 2.25 \\
\hline Rock type $^{\text {a }}$ & Granite & Granite & Granite & Granite & Granite & Granite & Granite & Granite & Granite \\
\hline Sample & 07081212 & 08010813 & 07010813 & 06010813 & 10020813 & 09020813 & 08020813 & 07020813 & 06020813 \\
\hline Longitude $^{\mathrm{b}}$ & $59^{\circ} 52^{\prime} 4^{\prime \prime}$ & $59^{\circ} 52^{\prime} 1^{\prime \prime}$ & $59^{\circ} 51^{\prime} 59^{\prime \prime}$ & $59^{\circ} 51^{\prime} 59^{\prime \prime}$ & $59^{\circ} 52^{\prime} 43^{\prime \prime}$ & $59^{\circ} 52^{\prime} 32^{\prime \prime}$ & $59^{\circ} 0^{\prime} 27^{\prime \prime}$ & $59^{\circ} 0^{\prime} 24^{\prime \prime}$ & $59^{\circ} 0^{\prime} 21^{\prime \prime}$ \\
\hline Latitude $^{\mathrm{b}}$ & $37^{\circ} 1^{\prime} 55^{\prime \prime}$ & $37^{\circ} 2^{\prime} 11^{\prime \prime}$ & $37^{\circ} 2^{\prime} 10^{\prime \prime}$ & $37^{\circ} 2^{\prime} 10^{\prime \prime}$ & $37^{\circ} 2^{\prime} 8^{\prime \prime}$ & $37^{\circ} 2^{\prime} 7^{\prime \prime}$ & $37^{\circ} 2^{\prime} 3^{\prime \prime}$ & $37^{\circ} 0^{\prime} 3^{\prime \prime}$ & $37^{\circ} 0^{\prime} 3^{\prime \prime}$ \\
\hline $\mathrm{SiO}_{2}$ & 76.83 & 77.52 & 77.42 & 76.96 & 78.64 & 76.97 & 77.96 & 77.86 & 77.29 \\
\hline $\mathrm{TiO}_{2}$ & 0.03 & 0.06 & 0.08 & 0.05 & 0.05 & 0.05 & 0.04 & 0.06 & 0.04 \\
\hline $\mathrm{Al}_{2} \mathrm{O}_{3}$ & 13.02 & 12.33 & 12.27 & 12.31 & 11.61 & 12.50 & 12.21 & 12.29 & 12.60 \\
\hline $\mathrm{FeO}$ & 0.75 & 1.16 & 1.42 & 1.18 & 1.08 & 1.31 & 1.10 & 1.08 & 1.30 \\
\hline $\mathrm{MgO}$ & 0.07 & 0.02 & 0.00 & 0.00 & 0.00 & 0.08 & 0.02 & 0.09 & 0.00 \\
\hline $\mathrm{MnO}$ & 0.02 & 0.01 & 0.01 & 0.01 & 0.01 & 0.02 & 0.01 & 0.02 & 0.02 \\
\hline $\mathrm{CaO}$ & 0.56 & 0.49 & 0.40 & 0.52 & 0.41 & 0.47 & 0.48 & 0.42 & 0.58 \\
\hline $\mathrm{Na}_{2} \mathrm{O}$ & 3.14 & 2.24 & 2.24 & 2.05 & 2.09 & 2.33 & 2.44 & 2.38 & 2.38 \\
\hline $\mathrm{K}_{2} \mathrm{O}$ & 4.92 & 5.35 & 5.25 & 5.52 & 5.26 & 5.53 & 5.03 & 5.17 & 5.10 \\
\hline $\mathrm{P}_{2} \mathrm{O}_{5}$ & 0.02 & 0.02 & 0.02 & 0.04 & 0.01 & 0.02 & 0.01 & 0.02 & 0.01 \\
\hline LOI & 0.37 & 0.44 & 0.48 & 0.89 & 0.39 & 0.27 & 0.28 & 0.25 & 0.24 \\
\hline Total & 99.72 & 100.93 & 101.17 & 100.84 & 100.75 & 101.01 & 100.80 & 100.83 & 101.01 \\
\hline
\end{tabular}


Table 1 (continued)

\begin{tabular}{|c|c|c|c|c|c|c|c|c|c|}
\hline $\mathrm{Li}$ & 5.93 & 4.70 & 6.56 & 3.57 & 2.23 & 4.00 & 4.64 & 4.39 & 5.38 \\
\hline $\mathrm{Be}$ & 0.87 & 1.14 & 0.89 & 1.22 & 0.67 & 1.14 & 0.57 & 0.71 & 1.77 \\
\hline $\mathrm{Sc}$ & 1.51 & 9.10 & 10.02 & 9.09 & 10.00 & 7.53 & 7.94 & 9.13 & 8.08 \\
\hline V & 6.12 & 24.42 & 25.34 & 25.98 & 28.98 & 23.81 & 18.90 & 23.57 & 23.97 \\
\hline $\mathrm{Cr}$ & 146.11 & 335.40 & 340.51 & 349.28 & 406.39 & 308.95 & 260.58 & 344.14 & 341.17 \\
\hline Co & 50.17 & 0.91 & 1.10 & 1.05 & 0.87 & 0.88 & 0.90 & 0.75 & 0.82 \\
\hline $\mathrm{Ni}$ & 5.25 & 9.81 & 9.91 & 9.75 & 10.61 & 8.93 & 7.82 & 9.52 & 9.36 \\
\hline $\mathrm{Cu}$ & 36.67 & 6.32 & 7.83 & 7.11 & 11.04 & 6.17 & 7.97 & 7.28 & 5.77 \\
\hline $\mathrm{Zn}$ & 20.08 & 14.08 & 15.96 & 12.20 & 13.24 & 15.06 & 12.80 & 13.75 & 14.47 \\
\hline $\mathrm{Ga}$ & 24.22 & 15.69 & 16.01 & 17.20 & 15.60 & 16.36 & 17.57 & 16.28 & 18.20 \\
\hline $\mathrm{Rb}$ & 211.54 & 198.74 & 210.06 & 222.57 & 210.43 & 174.22 & 206.03 & 160.08 & 212.95 \\
\hline $\mathrm{Sr}$ & 8.44 & 35.66 & 19.41 & 33.60 & 28.09 & 13.76 & 8.03 & 8.83 & 12.55 \\
\hline $\mathrm{Y}$ & 20.03 & 2.45 & 5.61 & 2.34 & 8.81 & 2.66 & 32.52 & 1.36 & 1.62 \\
\hline $\mathrm{Zr}$ & 76.10 & 109.00 & 116.40 & 101.00 & 91.70 & 94.10 & 81.80 & 92.20 & 148.00 \\
\hline $\mathrm{Nb}$ & 5.68 & 3.26 & 6.50 & 4.77 & 2.90 & 1.77 & 1.54 & 2.01 & 3.52 \\
\hline Cs & 0.14 & 0.39 & 0.28 & 0.26 & 0.53 & 0.34 & 0.34 & 0.47 & 1.20 \\
\hline $\mathrm{Ba}$ & 36.44 & 160.53 & 119.87 & 95.09 & 156.87 & 35.29 & 34.44 & 13.01 & 27.26 \\
\hline $\mathrm{La}$ & 6.53 & 10.22 & 7.88 & 11.58 & 7.51 & 5.14 & 5.47 & 2.33 & 3.94 \\
\hline $\mathrm{Ce}$ & 15.94 & 16.34 & 15.68 & 18.30 & 8.94 & 9.45 & 14.01 & 3.22 & 12.52 \\
\hline $\operatorname{Pr}$ & 2.16 & 1.88 & 1.77 & 2.25 & 1.29 & 0.97 & 1.77 & 0.23 & 0.78 \\
\hline $\mathrm{Nd}$ & 10.96 & 7.39 & 7.81 & 8.79 & 5.45 & 4.30 & 9.90 & 1.46 & 3.42 \\
\hline $\mathrm{Sm}$ & 4.34 & 1.29 & 1.80 & 1.53 & 1.41 & 0.80 & 3.82 & 0.21 & 0.54 \\
\hline $\mathrm{Eu}$ & 0.11 & 0.28 & 0.23 & 0.20 & 0.19 & 0.09 & 0.08 & 0.04 & 0.05 \\
\hline Gd & 4.32 & 0.87 & 1.44 & 0.96 & 1.47 & 0.59 & 4.38 & 0.21 & 0.39 \\
\hline $\mathrm{Tb}$ & 0.76 & 0.10 & 0.22 & 0.11 & 0.23 & 0.08 & 0.76 & 0.04 & 0.05 \\
\hline Dy & 5.03 & 0.49 & 1.17 & 0.42 & 1.56 & 0.42 & 5.13 & 0.20 & 0.23 \\
\hline Ho & 1.06 & 0.09 & 0.24 & 0.08 & 0.34 & 0.09 & 1.15 & 0.05 & 0.05 \\
\hline $\mathrm{Er}$ & 2.98 & 0.23 & 0.55 & 0.21 & 0.92 & 0.23 & 3.04 & 0.14 & 0.15 \\
\hline $\mathrm{Tm}$ & 0.52 & 0.04 & 0.08 & 0.04 & 0.14 & 0.04 & 0.45 & 0.02 & 0.03 \\
\hline $\mathrm{Yb}$ & 3.31 & 0.25 & 0.45 & 0.23 & 0.79 & 0.26 & 2.58 & 0.17 & 0.19 \\
\hline $\mathrm{Lu}$ & 0.49 & 0.05 & 0.08 & 0.05 & 0.12 & 0.05 & 0.38 & 0.04 & 0.04 \\
\hline $\mathrm{Hf}$ & 3.07 & 3.14 & 3.61 & 3.53 & 4.16 & 3.49 & 3.07 & 3.35 & 5.91 \\
\hline $\mathrm{Ta}$ & 0.45 & 0.11 & 0.11 & 0.07 & 0.11 & 0.05 & 0.05 & 0.06 & 0.18 \\
\hline W & 0.00 & 0.00 & 0.00 & 0.00 & 0.00 & 0.00 & 0.00 & 0.00 & 0.00 \\
\hline $\mathrm{Pb}$ & 42.75 & 23.95 & 24.01 & 32.90 & 26.01 & 28.70 & 31.49 & 13.55 & 31.11 \\
\hline Th & 12.69 & 6.35 & 3.68 & 10.05 & 5.66 & 29.48 & 12.53 & 10.84 & 23.63 \\
\hline $\mathrm{U}$ & 7.66 & 0.68 & 0.84 & 1.21 & 1.42 & 4.46 & 3.74 & 1.75 & 6.73 \\
\hline Rock type $^{a}$ & Granite & Granite & Granite & Granite & Granite & Granite & Granite & Granite & Granite \\
\hline Sample & 05020813 & 03010813 & 04020813 & 04081212 & 10031105 & 02051211 & 01061211 & 04071211 & 04061211 \\
\hline Longitude $^{\mathrm{b}}$ & $59^{\circ} 0^{\prime} 21^{\prime \prime}$ & $59^{\circ} 52^{\prime} 4^{\prime \prime}$ & $59^{\circ} 52^{\prime} 32^{\prime \prime}$ & $59^{\circ} 52^{\prime} 3^{\prime \prime}$ & $59^{\circ} 51^{\prime} 59^{\prime \prime}$ & $59^{\circ} 52^{\prime} 40^{\prime \prime}$ & $59^{\circ} 52^{\prime} 21^{\prime \prime}$ & $59^{\circ} 51^{\prime} 36^{\prime \prime}$ & $59^{\circ} 52^{\prime} 2^{\prime \prime}$ \\
\hline Latitude $^{\mathrm{b}}$ & $37^{\circ} 0^{\prime} 6^{\prime \prime}$ & $37^{\circ} 1^{\prime} 54^{\prime \prime}$ & $37^{\circ} 2^{\prime} 5^{\prime \prime}$ & $37^{\circ} 2^{\prime} 1^{\prime \prime}$ & $37^{\circ} 1^{\prime} 58^{\prime \prime}$ & $37^{\circ} 2^{\prime} 14^{\prime \prime}$ & $37^{\circ} 2^{\prime} 12^{\prime \prime}$ & $37^{\circ} 1^{\prime} 53^{\prime \prime}$ & $37^{\circ} 2^{\prime} 0^{\prime \prime}$ \\
\hline $\mathrm{SiO}_{2}$ & 77.25 & 77.84 & 76.82 & 77.91 & 73.74 & 74.06 & 74.55 & 74.81 & 75.09 \\
\hline $\mathrm{TiO}_{2}$ & 0.04 & 0.04 & 0.04 & 0.03 & 0.01 & 0.15 & 0.18 & 0.03 & 0.10 \\
\hline $\mathrm{Al}_{2} \mathrm{O}_{3}$ & 12.47 & 12.24 & 12.71 & 13.51 & 14.56 & 13.32 & 12.88 & 13.24 & 13.42 \\
\hline $\mathrm{FeO}$ & 1.36 & 0.93 & 1.46 & 0.80 & 0.82 & 1.84 & 2.35 & 1.46 & 1.25 \\
\hline $\mathrm{MgO}$ & 0.00 & 0.05 & 0.01 & 0.01 & 0.05 & 0.63 & 0.15 & 0.02 & 0.03 \\
\hline $\mathrm{MnO}$ & 0.02 & 0.01 & 0.03 & 0.01 & 0.01 & 0.02 & 0.04 & 0.01 & 0.01 \\
\hline $\mathrm{CaO}$ & 0.60 & 0.42 & 0.79 & 2.07 & 0.87 & 0.70 & 1.18 & 0.38 & 0.54 \\
\hline $\mathrm{Na}_{2} \mathrm{O}$ & 2.48 & 2.23 & 2.54 & 3.73 & 4.17 & 3.67 & 2.46 & 3.36 & 3.28 \\
\hline $\mathrm{K}_{2} \mathrm{O}$ & 5.09 & 5.57 & 5.01 & 1.11 & 4.86 & 4.40 & 5.10 & 5.66 & 5.64 \\
\hline $\mathrm{P}_{2} \mathrm{O}_{5}$ & 0.04 & 0.02 & 0.01 & 0.02 & 0.03 & 0.02 & 0.04 & 0.01 & 0.01 \\
\hline LOI & 0.27 & 0.28 & 0.15 & 0.28 & 0.80 & 0.90 & 0.50 & 0.90 & 0.50 \\
\hline Total & 101.12 & 100.66 & 101.20 & 100.37 & 99.92 & 99.71 & 99.43 & 99.88 & 99.87 \\
\hline \multicolumn{10}{|c|}{ Trace elements (ppm) } \\
\hline $\mathrm{Li}$ & 5.00 & 5.27 & 10.92 & 3.45 & 0.00 & 0.00 & 0.00 & 0.00 & 0.00 \\
\hline $\mathrm{Be}$ & 1.01 & 0.57 & 0.84 & 3.14 & 2.00 & $<1$ & 1.00 & $<1$ & $<1$ \\
\hline $\mathrm{Sc}$ & 9.13 & 8.43 & 9.79 & 10.23 & $<1$ & 2.00 & 12.00 & $<1$ & $<1$ \\
\hline V & 23.62 & 26.71 & 28.82 & 26.96 & $<8$ & $<8$ & 9.00 & $<8$ & $<8$ \\
\hline $\mathrm{Cr}$ & 367.18 & 373.13 & 429.93 & 403.12 & $<0,002$ & $<0,002$ & $<0,002$ & $<0,002$ & $<0,002$ \\
\hline Co & 0.89 & 1.01 & 1.22 & 1.07 & 1.50 & 1.40 & 1.50 & 1.00 & 0.70 \\
\hline $\mathrm{Ni}$ & 10.43 & 10.51 & 12.25 & 12.19 & $<20$ & $<20$ & $<20$ & $<20$ & $<20$ \\
\hline $\mathrm{Cu}$ & 6.96 & 37.02 & 13.62 & 7.05 & 34.40 & 4.90 & 14.00 & 24.50 & 37.10 \\
\hline $\mathrm{Zn}$ & 16.70 & 16.47 & 31.43 & 14.39 & 19.00 & 23.00 & 59.00 & 5.00 & 15.00 \\
\hline $\mathrm{Ga}$ & 16.84 & 19.74 & 19.13 & 18.74 & 21.70 & 16.90 & 16.70 & 21.10 & 18.10 \\
\hline $\mathrm{Rb}$ & 197.83 & 223.19 & 206.99 & 31.51 & 264.10 & 113.80 & 137.50 & 227.80 & 223.50 \\
\hline $\mathrm{Sr}$ & 12.33 & 16.31 & 32.55 & 148.65 & 40.30 & 72.60 & 226.20 & 17.10 & 45.10 \\
\hline $\mathrm{Y}$ & 6.49 & 5.03 & 6.03 & 4.18 & 12.40 & 4.80 & 13.90 & 13.70 & 2.10 \\
\hline $\mathrm{Zr}$ & 87.00 & 88.80 & 115.10 & 119.20 & 50.90 & 147.40 & 232.00 & 91.00 & 20.40 \\
\hline $\mathrm{Nb}$ & 2.28 & 3.57 & 4.13 & 3.36 & 0.50 & 7.90 & 5.70 & 2.60 & 5.80 \\
\hline Cs & 0.29 & 0.92 & 0.63 & 0.17 & 4.00 & 0.30 & 0.20 & 0.30 & 1.00 \\
\hline $\mathrm{Ba}$ & 40.12 & 61.15 & 97.68 & 341.25 & 76.00 & 183.00 & 2306.00 & 35.00 & 120.00 \\
\hline $\mathrm{La}$ & 5.38 & 9.65 & 6.77 & 14.03 & 12.10 & 49.80 & 78.30 & 4.70 & 4.40 \\
\hline $\mathrm{Ce}$ & 10.50 & 14.16 & 10.81 & 22.56 & 9.60 & 92.90 & 150.00 & 6.40 & 5.20 \\
\hline $\operatorname{Pr}$ & 1.01 & 1.22 & 0.96 & 2.71 & 3.04 & 10.07 & 16.64 & 0.66 & 0.44 \\
\hline
\end{tabular}


Table 1 (continued)

\begin{tabular}{|c|c|c|c|c|c|c|c|c|c|}
\hline $\mathrm{Nd}$ & 4.87 & 4.74 & 4.23 & 9.80 & 10.00 & 34.40 & 58.80 & 2.50 & 1.10 \\
\hline $\mathrm{Sm}$ & 1.10 & 0.93 & 1.03 & 1.60 & 2.99 & 4.72 & 8.53 & 0.85 & 0.20 \\
\hline $\mathrm{Eu}$ & 0.09 & 0.08 & 0.19 & 0.46 & 0.18 & 0.57 & 1.34 & 0.05 & 0.19 \\
\hline Gd & 1.09 & 0.86 & 1.08 & 1.12 & 2.51 & 3.17 & 6.60 & 1.19 & 0.21 \\
\hline $\mathrm{Tb}$ & 0.17 & 0.13 & 0.16 & 0.15 & 0.44 & 0.25 & 0.68 & 0.30 & 0.04 \\
\hline Dy & 0.92 & 0.73 & 1.08 & 0.68 & 2.30 & 1.00 & 3.11 & 2.28 & 0.27 \\
\hline Ho & 0.19 & 0.16 & 0.23 & 0.14 & 0.42 & 0.13 & 0.54 & 0.55 & 0.05 \\
\hline $\mathrm{Er}$ & 0.42 & 0.44 & 0.62 & 0.37 & 1.18 & 0.30 & 1.41 & 1.88 & 0.18 \\
\hline $\mathrm{Tm}$ & 0.06 & 0.08 & 0.10 & 0.06 & 0.18 & 0.06 & 0.16 & 0.30 & 0.03 \\
\hline $\mathrm{Yb}$ & 0.37 & 0.42 & 0.61 & 0.36 & 1.06 & 0.31 & 0.95 & 1.86 & 0.23 \\
\hline $\mathrm{Lu}$ & 0.06 & 0.09 & 0.11 & 0.07 & 0.16 & 0.05 & 0.13 & 0.25 & 0.03 \\
\hline $\mathrm{Hf}$ & 2.89 & 4.81 & 5.49 & 4.85 & 2.00 & 5.00 & 5.90 & 5.70 & 1.10 \\
\hline $\mathrm{Ta}$ & 0.05 & 0.13 & 0.10 & 0.16 & $<0,1$ & 0.10 & $<0,1$ & $<0,1$ & 0.30 \\
\hline $\mathrm{W}$ & 0.00 & 0.00 & 0.00 & 0.00 & $<0,5$ & $<0,5$ & $<0,5$ & $<0,5$ & $<0,5$ \\
\hline $\mathrm{Pb}$ & 30.89 & 47.91 & 58.28 & 19.80 & 30.90 & 18.60 & 6.50 & 14.30 & 14.20 \\
\hline Th & 17.55 & 9.95 & 19.88 & 15.18 & 9.10 & 48.10 & 11.30 & 12.20 & 4.00 \\
\hline $\mathrm{U}$ & 2.57 & 2.15 & 5.06 & 1.91 & 3.60 & 1.20 & 0.50 & 2.90 & 0.90 \\
\hline Rock type $^{\mathrm{a}}$ & Granite & Granite & Granite & Granite & Granite & Granite & Granite & Granite & ICG \\
\hline Sample & 08270404 & 05270404 & 05071211 & 06270404 & 07270404 & 11061211 & 16061211 & 03230715 & 10081212 \\
\hline Longitude $^{\mathrm{b}}$ & $59^{\circ} 52^{\prime} 1^{\prime \prime}$ & $59^{\circ} 52^{\prime} 5^{\prime \prime}$ & $59^{\circ} 51^{\prime} 52^{\prime \prime}$ & $59^{\circ} 52^{\prime} 0^{\prime \prime}$ & $59^{\circ} 52^{\prime} 0^{\prime \prime}$ & $59^{\circ} 52^{\prime} 6^{\prime \prime}$ & $59^{\circ} 53^{\prime} 10^{\prime \prime}$ & $59^{\circ} 51^{\prime} 10^{\prime \prime}$ & $59^{\circ} 52^{\prime} 3^{\prime \prime}$ \\
\hline Latitude $^{\mathrm{b}}$ & $37^{\circ} 2^{\prime} 3^{\prime \prime}$ & $37^{\circ} 1^{\prime} 55^{\prime \prime}$ & $37^{\circ} 1^{\prime} 57^{\prime \prime}$ & $37^{\circ} 1^{\prime} 58^{\prime \prime}$ & $37^{\circ} 2^{\prime} 0^{\prime \prime}$ & $37^{\circ} 2^{\prime} 0^{\prime \prime}$ & $37^{\circ} 3^{\prime} 5^{\prime \prime}$ & $37^{\circ} 01^{\prime} 50^{\prime \prime}$ & $37^{\circ} 1^{\prime} 54^{\prime \prime}$ \\
\hline $\mathrm{SiO}_{2}$ & 75.55 & 77.21 & 77.24 & 77.66 & 77.98 & 78.04 & 78.67 & 74.90 & 68.80 \\
\hline $\mathrm{TiO}_{2}$ & 0.03 & 0.07 & 0.02 & 0.08 & 0.04 & 0.02 & 0.06 & 0.14 & 0.30 \\
\hline $\mathrm{Al}_{2} \mathrm{O}_{3}$ & 12.74 & 12.37 & 12.18 & 11.57 & 11.64 & 12.00 & 11.68 & 12.93 & 15.80 \\
\hline $\mathrm{FeO}$ & 1.36 & 0.88 & 1.35 & 0.88 & 1.27 & 1.22 & 0.74 & 2.29 & 3.44 \\
\hline $\mathrm{MgO}$ & 0.04 & 0.03 & 0.05 & 0.06 & 0.04 & 0.12 & 0.10 & 0.03 & 0.64 \\
\hline $\mathrm{MnO}$ & 0.02 & 0.01 & 0.02 & 0.01 & 0.01 & 0.01 & 0.01 & 0.05 & 0.09 \\
\hline $\mathrm{CaO}$ & 0.36 & 0.44 & 0.62 & 0.47 & 0.55 & 0.68 & 0.59 & 1.32 & 2.32 \\
\hline $\mathrm{Na}_{2} \mathrm{O}$ & 2.71 & 3.37 & 3.53 & 2.91 & 3.19 & 3.49 & 2.94 & 3.06 & 3.29 \\
\hline $\mathrm{K}_{2} \mathrm{O}$ & 6.05 & 4.95 & 4.39 & 5.16 & 4.59 & 3.85 & 4.71 & 4.37 & 3.95 \\
\hline $\mathrm{P}_{2} \mathrm{O}_{5}$ & 0.02 & 0.02 & 0.01 & 0.02 & 0.02 & 0.02 & 0.01 & 0.04 & 0.09 \\
\hline LOI & 1.00 & 0.50 & 0.40 & 1.00 & 0.50 & 0.40 & 0.40 & 0.00 & 0.50 \\
\hline Total & 99.88 & 99.85 & 99.81 & 99.82 & 99.83 & 99.85 & 99.91 & 99.39 & 99.21 \\
\hline \multicolumn{10}{|c|}{ Trace elements (ppm) } \\
\hline $\mathrm{Li}$ & 0.00 & 0.00 & 0.00 & 0.00 & 0.00 & 0.00 & 0.00 & 30.24 & 48.21 \\
\hline $\mathrm{Be}$ & $<1$ & $<1$ & 1.00 & $<1$ & $<1$ & $<1$ & $<1$ & 2.72 & 4.52 \\
\hline Sc & $<1$ & $<1$ & 1.00 & $<1$ & $<1$ & $<1$ & $<1$ & 14.46 & 11.07 \\
\hline $\mathrm{V}$ & $<8$ & $<8$ & $<8$ & $<8$ & $<8$ & $<8$ & $<8$ & 46.96 & 31.52 \\
\hline $\mathrm{Cr}$ & $<0,002$ & $<0,002$ & $<0,002$ & $<0,002$ & $<0,002$ & $<0,002$ & $<0,002$ & 447.53 & 254.80 \\
\hline Co & 1.00 & 0.70 & 0.80 & 0.80 & 0.70 & 0.70 & 0.70 & 12.36 & 55.16 \\
\hline $\mathrm{Ni}$ & $<20$ & $<20$ & $<20$ & $<20$ & $<20$ & $<20$ & $<20$ & 203.45 & 10.52 \\
\hline $\mathrm{Cu}$ & 12.50 & 6.10 & 12.40 & 4.20 & 6.90 & 22.10 & 6.40 & 23.35 & 22.53 \\
\hline $\mathrm{Zn}$ & 16.00 & 5.00 & 30.00 & 4.00 & 10.00 & 5.00 & 4.00 & 109.40 & 73.55 \\
\hline $\mathrm{Ga}$ & 16.30 & 16.40 & 19.30 & 15.30 & 19.40 & 19.10 & 13.80 & 23.19 & 23.78 \\
\hline $\mathrm{Rb}$ & 205.10 & 161.80 & 232.80 & 176.20 & 233.40 & 158.90 & 132.00 & 191.82 & 355.06 \\
\hline $\mathrm{Sr}$ & 16.80 & 51.70 & 15.30 & 50.50 & 18.60 & 19.30 & 45.40 & 440.42 & 421.61 \\
\hline $\mathrm{Y}$ & 1.40 & 3.60 & 100.90 & 3.70 & 30.70 & 4.30 & 1.60 & 41.74 & 25.86 \\
\hline $\mathrm{Zr}$ & 70.20 & 101.00 & 102.50 & 99.30 & 88.40 & 21.30 & 93.00 & 295.10 & 215.20 \\
\hline $\mathrm{Nb}$ & 0.60 & 1.80 & 2.20 & 1.30 & 3.30 & 2.10 & 0.90 & 24.29 & 17.50 \\
\hline Cs & 0.30 & 0.40 & $<0,1$ & 0.50 & 0.40 & 0.30 & 0.40 & 2.21 & 5.26 \\
\hline $\mathrm{Ba}$ & 45.00 & 136.00 & 28.00 & 133.00 & 88.00 & 33.00 & 112.00 & 1873.11 & 2977.14 \\
\hline $\mathrm{La}$ & 6.50 & 20.10 & 13.00 & 13.20 & 7.70 & 6.30 & 10.20 & 54.28 & 56.35 \\
\hline $\mathrm{Ce}$ & 7.80 & 36.30 & 32.50 & 15.90 & 19.10 & 12.00 & 12.50 & 147.01 & 110.73 \\
\hline Pr & 0.63 & 3.28 & 4.84 & 1.76 & 1.74 & 1.24 & 1.11 & 16.37 & 12.25 \\
\hline $\mathrm{Nd}$ & 1.70 & 10.40 & 24.10 & 6.90 & 8.00 & 4.40 & 3.40 & 67.30 & 44.46 \\
\hline $\mathrm{Sm}$ & 0.26 & 1.49 & 9.89 & 0.87 & 3.12 & 0.94 & 0.40 & 13.87 & 8.08 \\
\hline $\mathrm{Eu}$ & 0.14 & 0.22 & 0.07 & 0.23 & 0.08 & 0.06 & 0.18 & 1.89 & 1.51 \\
\hline $\mathrm{Gd}$ & 0.30 & 1.22 & 12.03 & 0.69 & 3.98 & 0.99 & 0.39 & 10.53 & 6.05 \\
\hline $\mathrm{Tb}$ & 0.03 & 0.12 & 2.10 & 0.10 & 0.85 & 0.13 & 0.04 & 1.48 & 0.91 \\
\hline Dy & 0.17 & 0.68 & 12.27 & 0.56 & 5.58 & 0.77 & 0.28 & 7.70 & 5.02 \\
\hline Ho & 0.04 & 0.12 & 2.48 & 0.11 & 1.21 & 0.15 & 0.04 & 1.48 & 0.99 \\
\hline Er & 0.16 & 0.28 & 7.37 & 0.34 & 3.87 & 0.43 & 0.19 & 3.57 & 2.66 \\
\hline $\mathrm{Tm}$ & 0.03 & 0.05 & 0.97 & 0.06 & 0.60 & 0.06 & 0.03 & 0.54 & 0.44 \\
\hline $\mathrm{Yb}$ & 0.13 & 0.39 & 6.23 & 0.41 & 3.30 & 0.37 & 0.21 & 3.16 & 2.64 \\
\hline $\mathrm{Lu}$ & 0.04 & 0.07 & 0.84 & 0.08 & 0.46 & 0.05 & 0.03 & 0.46 & 0.41 \\
\hline $\mathrm{Hf}$ & 2.90 & 5.20 & 6.10 & 5.10 & 5.10 & 1.00 & 4.50 & 6.89 & 4.25 \\
\hline $\mathrm{Ta}$ & $<0,1$ & $<0,1$ & $<0,1$ & 0.10 & $<0,1$ & $<0,1$ & $<0,1$ & 1.41 & 1.88 \\
\hline $\mathrm{W}$ & $<0,5$ & $<0,5$ & $<0,5$ & $<0,5$ & $<0,5$ & $<0,5$ & $<0,5$ & 0.00 & 0.00 \\
\hline $\mathrm{Pb}$ & 16.30 & 9.00 & 19.10 & 7.10 & 10.00 & 5.10 & 2.00 & 23.40 & 25.02 \\
\hline Th & 3.60 & 10.50 & 12.20 & 3.10 & 19.80 & 2.60 & 1.10 & 12.42 & 13.31 \\
\hline $\mathrm{U}$ & 0.80 & 1.20 & 5.30 & 1.10 & 7.80 & 1.30 & 0.50 & 2.10 & 2.67 \\
\hline Rock type $^{\mathrm{a}}$ & \multicolumn{2}{|c|}{ ICG } & ICG & \multicolumn{2}{|l|}{ ICG } & ICG & \multicolumn{2}{|c|}{ Granulite } & Granodiorita \\
\hline Sample & \multicolumn{2}{|c|}{04010813} & 03020813 & \multicolumn{2}{|c|}{02270404} & 03270404 & \multicolumn{2}{|c|}{14031105} & $\begin{array}{l}03081212 \\
\text { on next page) }\end{array}$ \\
\hline
\end{tabular}


Table 1 (continued)

\begin{tabular}{|c|c|c|c|c|c|c|}
\hline Rock type $^{\mathrm{a}}$ & ICG & ICG & ICG & ICG & Granulite & Granodiorita \\
\hline Longitude $^{\mathrm{b}}$ & $59^{\circ} 52^{\prime} 4^{\prime \prime}$ & $59^{\circ} 52^{\prime} 32^{\prime \prime}$ & $59^{\circ} 52^{\prime} 5^{\prime \prime}$ & $59^{\circ} 52^{\prime} 5^{\prime \prime}$ & $59^{\circ} 51^{\prime} 57^{\prime \prime}$ & $59^{\circ} 52^{\prime} 12^{\prime \prime}$ \\
\hline Latitude $^{\mathrm{b}}$ & $37^{\circ} 1^{\prime} 54^{\prime \prime}$ & $37^{\circ} 2^{\prime} 5^{\prime \prime}$ & $37^{\circ} 1^{\prime} 53^{\prime \prime}$ & $37^{\circ} 1^{\prime} 53^{\prime \prime}$ & $37^{\circ} 2^{\prime} 20^{\prime \prime}$ & $37^{\circ} 2^{\prime} 7^{\prime \prime}$ \\
\hline $\mathrm{SiO}_{2}$ & 58.90 & 60.85 & 64.16 & 69.60 & 57.81 & 63.63 \\
\hline $\mathrm{TiO}_{2}$ & 0.80 & 0.51 & 0.51 & 0.23 & 0.73 & 0.98 \\
\hline $\mathrm{Al}_{2} \mathrm{O}_{3}$ & 16.39 & 18.97 & 15.85 & 15.32 & 18.26 & 15.34 \\
\hline $\mathrm{FeO}$ & 7.54 & 4.90 & 4.46 & 2.95 & 6.85 & 6.46 \\
\hline $\mathrm{MgO}$ & 3.30 & 1.03 & 1.65 & 0.20 & 2.90 & 1.22 \\
\hline $\mathrm{MnO}$ & 0.15 & 0.10 & 0.09 & 0.05 & 0.07 & 0.10 \\
\hline $\mathrm{CaO}$ & 4.80 & 5.17 & 4.03 & 1.91 & 6.33 & 2.94 \\
\hline $\mathrm{Na}_{2} \mathrm{O}$ & 3.18 & 4.35 & 3.08 & 3.57 & 3.16 & 3.48 \\
\hline $\mathrm{K}_{2} \mathrm{O}$ & 2.10 & 2.08 & 3.88 & 4.59 & 1.28 & 3.82 \\
\hline $\mathrm{P}_{2} \mathrm{O}_{5}$ & 0.40 & 0.27 & 0.21 & 0.04 & 0.35 & 0.42 \\
\hline LOI & 1.02 & 0.55 & 1.30 & 1.00 & 1.10 & 0.42 \\
\hline Total & 106.96 & 104.22 & 99.22 & 99.46 & 98.84 & 99.55 \\
\hline \multicolumn{7}{|c|}{ Trace elements (ppm) } \\
\hline $\mathrm{Li}$ & 75.00 & 23.08 & 0.00 & 0.00 & 0.00 & 15.08 \\
\hline $\mathrm{Be}$ & 3.38 & 2.43 & 3.00 & 2.00 & 2.00 & 1.49 \\
\hline $\mathrm{Sc}$ & 18.26 & 12.78 & 9.00 & 10.00 & 16.00 & 7.74 \\
\hline V & 118.51 & 40.81 & 56.00 & 8.00 & 79.00 & 11.48 \\
\hline $\mathrm{Cr}$ & 336.68 & 168.65 & 0.00 & $<0,002$ & 0.00 & 1762.15 \\
\hline Co & 17.92 & 8.68 & 9.50 & 1.90 & 17.60 & 11.79 \\
\hline $\mathrm{Ni}$ & 24.70 & 10.50 & $<20$ & $<20$ & 26.00 & 818.09 \\
\hline $\mathrm{Cu}$ & 113.15 & 6.90 & 18.80 & 11.80 & 10.10 & 45.09 \\
\hline Zn & 137.89 & 77.99 & 68.00 & 47.00 & 58.00 & 25.74 \\
\hline $\mathrm{Ga}$ & 23.23 & 22.64 & 17.30 & 18.60 & 20.20 & 18.31 \\
\hline $\mathrm{Rb}$ & 210.52 & 124.34 & 171.40 & 133.20 & 72.10 & 143.19 \\
\hline $\mathrm{Sr}$ & 780.38 & 743.51 & 671.60 & 314.10 & 818.70 & 181.77 \\
\hline $\mathrm{Y}$ & 81.92 & 21.35 & 18.80 & 12.10 & 15.80 & 15.11 \\
\hline $\mathrm{Zr}$ & 162.80 & 106.60 & 177.40 & 274.20 & 135.70 & 136.43 \\
\hline $\mathrm{Nb}$ & 23.22 & 10.12 & 8.90 & 6.40 & 6.60 & 5.56 \\
\hline Cs & 6.40 & 2.01 & 3.00 & 0.60 & 2.70 & 1.36 \\
\hline $\mathrm{Ba}$ & 1186.69 & 897.47 & 1479.00 & 1643.00 & 1020.00 & 1880.01 \\
\hline $\mathrm{La}$ & 59.94 & 27.86 & 48.70 & 45.80 & 35.90 & 55.11 \\
\hline $\mathrm{Ce}$ & 131.16 & 61.22 & 99.10 & 106.00 & 75.40 & 102.90 \\
\hline $\operatorname{Pr}$ & 16.64 & 7.31 & 11.42 & 10.03 & 9.17 & 10.80 \\
\hline $\mathrm{Nd}$ & 67.32 & 29.71 & 40.60 & 37.00 & 37.60 & 37.76 \\
\hline $\mathrm{Sm}$ & 15.39 & 6.14 & 6.77 & 5.49 & 5.95 & 5.46 \\
\hline $\mathrm{Eu}$ & 1.82 & 1.55 & 1.48 & 1.47 & 1.75 & 0.94 \\
\hline Gd & 14.02 & 4.80 & 4.33 & 3.67 & 4.31 & 3.60 \\
\hline $\mathrm{Tb}$ & 2.21 & 0.69 & 0.66 & 0.50 & 0.60 & 0.48 \\
\hline Dy & 13.49 & 3.61 & 3.25 & 2.49 & 2.97 & 2.44 \\
\hline Ho & 2.78 & 0.70 & 0.63 & 0.43 & 0.53 & 0.53 \\
\hline $\mathrm{Er}$ & 7.43 & 1.81 & 1.81 & 1.14 & 1.50 & 1.44 \\
\hline $\mathrm{Tm}$ & 1.14 & 0.28 & 0.29 & 0.19 & 0.24 & 0.23 \\
\hline $\mathrm{Yb}$ & 6.96 & 1.80 & 1.85 & 1.12 & 1.57 & 1.46 \\
\hline $\mathrm{Lu}$ & 1.02 & 0.28 & 0.28 & 0.18 & 0.25 & 0.23 \\
\hline $\mathrm{Hf}$ & 4.64 & 2.63 & 4.60 & 7.00 & 3.10 & 3.68 \\
\hline $\mathrm{Ta}$ & 1.51 & 0.57 & 0.90 & 0.10 & 0.30 & 0.36 \\
\hline $\mathrm{W}$ & 0.00 & 0.00 & $<0,5$ & $<0,5$ & $<0,5$ & 0.00 \\
\hline $\mathrm{Pb}$ & 23.06 & 21.19 & 4.50 & 5.10 & 7.00 & 26.78 \\
\hline Th & 13.10 & 6.58 & 9.40 & 8.50 & 3.50 & 12.98 \\
\hline $\mathrm{U}$ & 4.24 & 1.92 & 1.90 & 0.90 & 0.70 & 0.87 \\
\hline
\end{tabular}

${ }^{\text {a }}$ FCG: Felsic Composition Gneiss; ICG: Intermediate Composition Gneiss.

b Geographical coordinates in degrees, minutes, seconds. (Datum of coordinates: WGS 84).

patterns. All SA rocks display $\mathrm{Nb}$ negative anomalies. All other rocks, except for amphibolite, granulite and granodiorite, present considerable negative anomalies for $\mathrm{P}$, possibly conditioned by the presence of apatite, an abundant accessory phase in amphibolite and granulite compositions. The negative anomaly of $\mathrm{Ti}$ is more pronounced in the most differentiated rocks, such as granite and felsic gneiss. Except for amphibolite and granulite, all rocks show negative and progressive $\mathrm{Sr}$ anomalies, due to the existence of a plagioclase-rich source. The LIL mobile elements group shows a contrast in behavior, which is more evident in granitic rocks. In the trace element distribution diagrams normalized with primitive mantle values (Sun and McDonough, 1989) negative $\mathrm{Nb}$, Ta and $\mathrm{Ti}$ anomalies are displayed (Fig. 11). Most of the rocks exhibit positive peaks in elements $\mathrm{Pb}, \mathrm{Zr}$ y $\mathrm{Sr}$. In particular, the $\mathrm{Zr}$ pattern is a response brought about by zircon abundance, being flat in rock with scarce zircons, like amphibolites, and indicating modest positive anomalies in rocks richer in these minerals, such as gneisses, granites and granodiorites. The primitive mantle-normalized multi-element pattern for average Archean TTG is represented by a red line while the grey field represents the compositional field for sanukitoids (Martin et al., 2009) in the multi-element diagram for amphibolite in Fig. 11. The similarity between amphibolite and sanukitoid patterns is remarkable, especially as regards negative anomalies of $\mathrm{Nb}$ and $\mathrm{Ti}$.

$\mathrm{Nb}, \mathrm{Y}, \mathrm{Ta}$ and $\mathrm{Yb}$ trace-element values for all SA samples, as well as previously-published basement analyses from Tandilia (Di Paola and Espiasse, 1986; Dalla Salda et al., 1992; Di Paola, 1995; Iacumin et al., 2001; Frisicale et al., 2005; Martínez and Dristas, 2007; Jorgensen et al., 2008; Frisicale et al., 2012; Massonne et al., 2012), were plotted on the tectonic discrimination diagrams devised by Pearce et al. (1984), showing similar values for volcanic arc and syn-collisional granitoids (Fig. 12). 

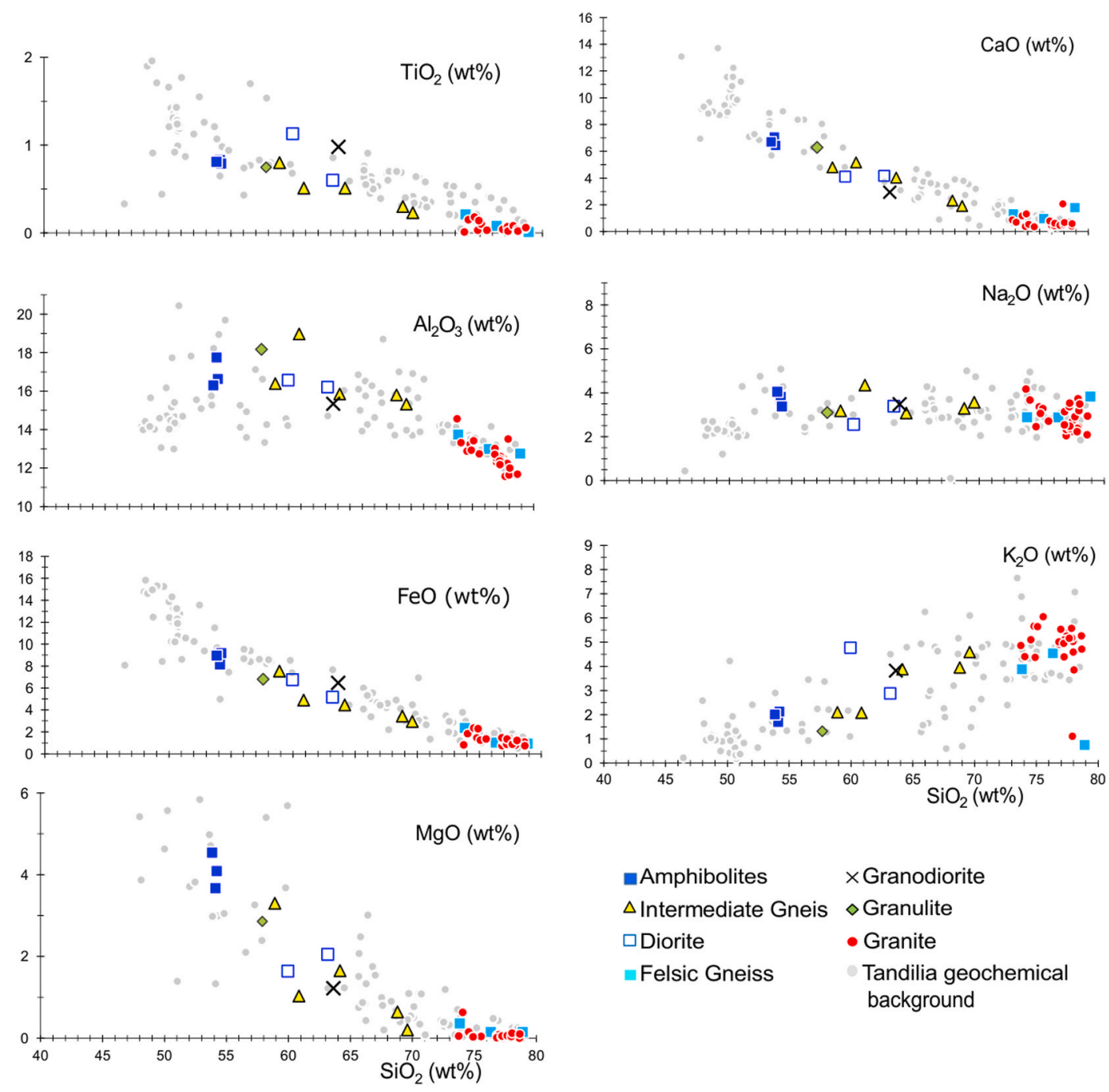

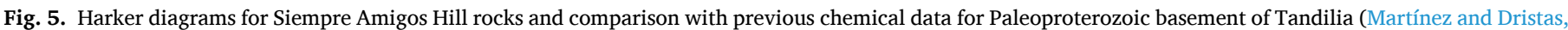

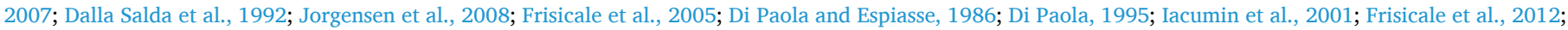
Massonne et al., 2012).

\section{3. $U-P b$ zircon geochronology}

\subsubsection{Granodiorite (sample 0308)}

$\mathrm{U}-\mathrm{Th}-\mathrm{Pb}$ isotopic analyses for 39 areas of 33 representative zircon grains from granodiorite Sample 0308 were obtained (Table 2). Most zircons are $100-180 \mu \mathrm{m}$ in diameter, simple and composite, subhedral prisms. Some zircons show crystal faces rounded, probably due to solubility effects. CL imaging (Fig. 13) showed that the vast majority of grains had oscillatory concentric zoning (Analyses 1.1, 2.1, 4.1, 32.1) but some presented slight or weak oscillatory zoning $(3.1,5.1)$. Four zircons were elongated and showed longitudinal banded zoning (an. 18.1).

The 39 analyses revealed a range of values for $\mathrm{U}(47.9-1235.8 \mathrm{ppm})$ and Th (19.3-847.5 ppm), while it showed a uniform $\mathrm{Th} / \mathrm{U}$ ratio $(0.14-0.84)$ with an average of 0.47 , consistent with precipitation from the melt phase of felsic magma. The appearance of some zircons suggests that they are composite, with two apparent growth phases $(13,19,21$, $23,31)$. Some present a very low luminescent apparent overgrowth with a relatively high $U$ content $(13,21)$. The two phases are similar in age (for example: 13, 21) and age distributions along the discordia line (Fig. 14 a) do not clearly reveal two age clusters, which might suggest an additional intra-Paleoproterozoic igneous or metamorphic event and/or a process of complex magmatic evolution characterized by an initial stage of zircon crystallization, a transient period of dissolution/resorption, and a final stage of zircon crystallization. Apart from analyses 14.1, 15.1 and 22.1, the other $36 \mathrm{U}-\mathrm{Pb}$ isotopic compositions are distributed along a defined discordia line (Fig. 14a) with an upper intersection in the concordia curve at $2121 \pm 35 \mathrm{Ma}(\mathrm{MSWD}=53$ ). The scatter is essentially associated with isotopic perturbation, promoting differential loss of radiogenic $\mathrm{Pb}$ and $\mathrm{Th}$ from an initial single igneous source. The lower intercept at $318 \pm 91 \mathrm{Ma}$ (Fig. 14a) does not appear to be correlated with a known geological event.

The weighted average for all eighteen analyses with discordance of $\leq 5 \%$, yielded a weighted mean $207 \mathrm{~Pb} / 206 \mathrm{~Pb}$ age of $2099 \pm 16 \mathrm{Ma}$ at a lower MSWD value $=16$. Taking the minor excess scatter omitting two (18.1, and 5.1) of these 18 analyses, the remaining $16207 \mathrm{~Pb} / 206 \mathrm{~Pb}$ ages were the same within the range of analytical uncertainty, with a well-defined weighted mean age of $2092 \pm 12 \mathrm{Ma}(\mathrm{MSWD}=7.6 ; \mathrm{n}=16$ ) (Fig. 14a). Despite a relatively high MSWD (which may be a result of recent $\mathrm{Pb}$ loss), $2092 \pm 13 \mathrm{Ma}$ (Rhyacian) is representative of the sample, and therefore is the best conservative estimate for the igneous protolith crystallization age of the granite. Analysis 15.1 yields a Neoarchaean age of $2.7 \mathrm{Ga}$, interpreted as the only inherited zircon age.

\subsubsection{Gneiss of intermediate composition (sample 1008)}

$\mathrm{U}-\mathrm{Th}-\mathrm{Pb}$ isotopic analyses of 31 representative zircon grains from 

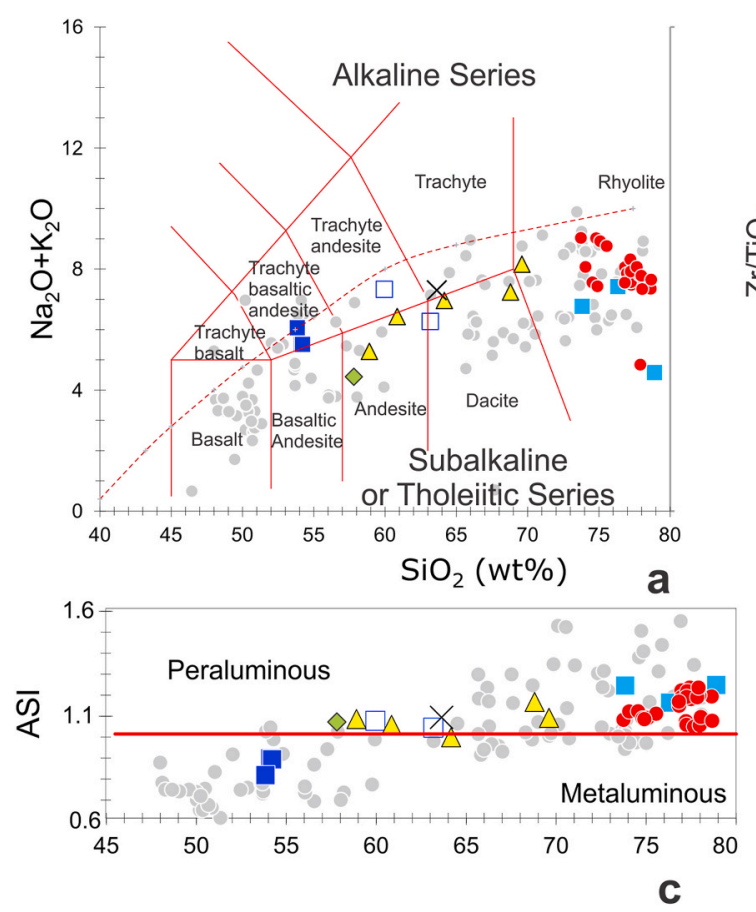

$\begin{array}{ll}\square \text { Amphibolites } & \times \text { Granodiorite } \\ \triangle \text { Intermediate Gneis } & \diamond \text { Granulite } \\ \square \text { Diorite } & \bullet \text { Granite } \\ \square \text { Felsic Gneiss } & \text { Tandilia geochemical background }\end{array}$
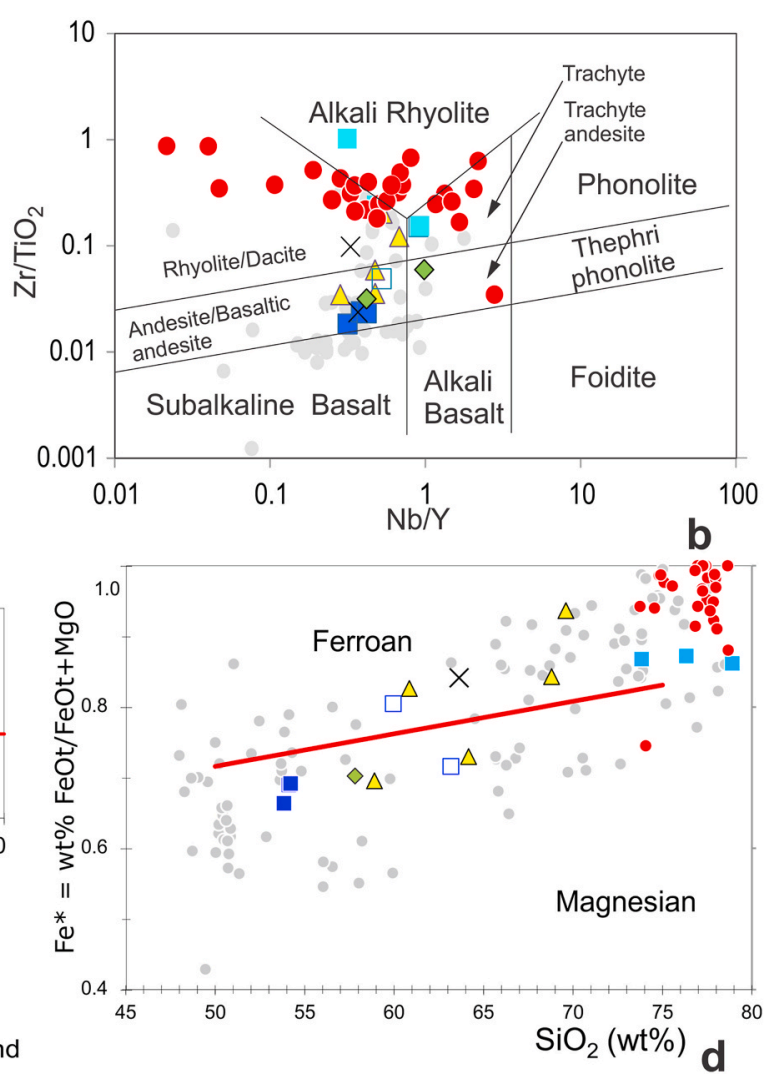

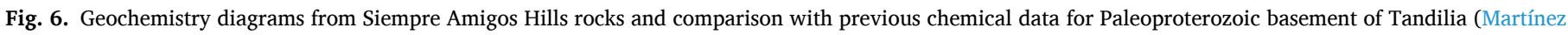

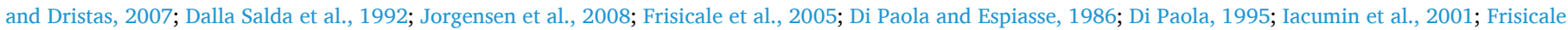

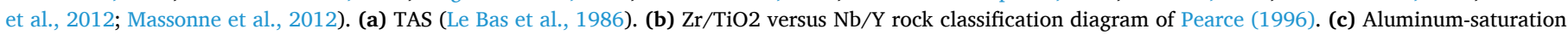

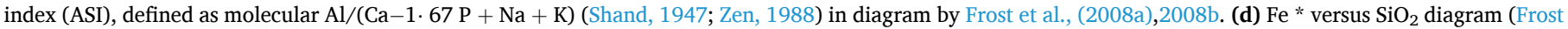
et al., 2001), $\mathrm{Fe}$ * defined as $\mathrm{wt} \% \mathrm{FeO}_{\mathrm{t}} / \mathrm{FeO}_{\mathrm{t}}+\mathrm{MgO}$.

intermediate composition gneiss Sample 1008 are listed in Table 2.

Except for one crystal which is $200 \mu \mathrm{m}$ long, all 28 analyzed zircons occurred as fine-to medium-grained $(30-100 \mu \mathrm{m})$, and are mostly noncomposite subhedral prisms, some of them presenting rounded crystal faces (Fig. 13). Unzoned and faintly concentric-zoned zircons (9.1; 17.1; $19.1 ; 30.1)$ constitute the most prominent zircon population, while there is also a group of banded or longitudinal-zoned zircons with a higher elongation ratio $(2.1 ; 5.1 ; 6.1 ; 11.1 ; 13.1)$.

$\mathrm{U}-\mathrm{Th}-\mathrm{Pb}$ isotopic analysis showed moderate to high $\mathrm{U}$ content $(82.7-2875.5 \mathrm{ppm}$; average $=614)$ and $\mathrm{Th} / \mathrm{U}$ ratios ranging from 0.11 to 0.94 with an average $=0.48$, similar to the Th/ $\mathrm{U}$ ratio obtained for Sample 0308. Apart from analyses 13.1 and 26.1, which presented a high proportion of $206 / 238$ and $207 / 235$ errors, the remaining 29 values plot along a potential discordia line that intercepts the concordia curve at $2123 \pm 16 \mathrm{Ma}$ (MSWD = 5.7) (Fig. 14b).

The lower intercept at $409 \pm 77 \mathrm{Ma}$ is within the range of error of the granodiorite lower intercept age, but its geological meaning remains unclear. Since it is not possible to obtain a concordia age, a relatively well-defined weighted mean $207 \mathrm{~Pb} / 206 \mathrm{~Pb}$ age of $2104.3 \pm 7.6 \mathrm{Ma}$ (MSWD $=6.4, \mathrm{n}=15$ ) was obtained by pooling all fifteen analyses with a positive discordance $\leq 10 \%$, which is a valid approach for obtaining the Rhyacian crystallization age of the igneous protolith.

\section{4. $\mathrm{Lu}-\mathrm{Hf}$ isotopes}

Granodiorite and intermediate gneiss samples were selected for $\mathrm{Lu}-\mathrm{Hf}$ analysis, based on their lowest $\mathrm{U} / \mathrm{Pb}$ age discordance percentage. 27 analyses were performed on 25 zircons selected from granodiorite (Sample 0308), and 16 analyses were performed in the same number of crystals from intermediate gneiss (Sample 1008) (Table 3).
As illustrated in Fig. 15a, SA rocks present both positive and negative $\varepsilon_{\mathrm{Hf}}(\mathrm{t})$ values. The ${ }^{176} \mathrm{Hf} /{ }^{177} \mathrm{Hf}$ ratios obtained for granodiorite range from 0.281330 to 0.281670 and correspond to $\varepsilon \mathrm{Hf}_{(\mathrm{t})}$ value ranges from -4.68 to +16.96 (Table 3; Fig. 15a). For gneiss, ${ }^{176} \mathrm{Hf} /{ }^{177} \mathrm{Hf}$ values range from 0.281290 to 0.281560 with $\varepsilon \mathrm{Hf}_{(\mathrm{t})}$ results ranging from -5.54 to 2.31 (Table 3; Fig. 15a). The calculated $\mathrm{Hf}_{\mathrm{DM}}$ ages, that is the age of the protolith differentiation from the mantle, range from $2.3 \mathrm{Ga}$ to 2.7 Ga for granodiorite and 2.4 to $2.8 \mathrm{Ga}$ for gneiss. The two lithologies show an average value of $2.5 \mathrm{Ga}$ (Table 2). At the same time, $\mathrm{T}_{\mathrm{DM}}^{\mathrm{C}}$ age, also termed cortical model age, assumes that the source of magma was derived from an average continental crust that was initially extracted from depleted mantle. The minimum $\mathrm{T}_{\mathrm{DM}}^{\mathrm{C}}$ value, $2.8 \mathrm{Ga}$, is almost coincident with the maximum $\mathrm{Hf} \mathrm{T}_{\mathrm{DM}}$ value, and very similar to the value of the only inherited core of granodiorite (spot $15.1=2771 \mathrm{Ma}$ ).

The magmatic ages of the two samples occur in the form of a coherent cluster around 2-2.1 Ga (Fig. 15b; Table 3). Only one zircon crystal from the granodiorite sample, the single inherited grain mentioned above, shows a Neoarchaean age of $2.7 \mathrm{Ga}$, similar to the maximum $\mathrm{Hf}$ model age of $2.8 \mathrm{Ga}$ (Fig. 15b). In order to calculate this, $\mathrm{a}^{176} \mathrm{Lu} /{ }^{177} \mathrm{Hf}$ ratio with a value of 0.0113 was used for the crust average (Taylor and McLennan, 1985; Wedepohl, 1995).

As is shown in Fig. 15, the results for zircon crystals from all SA rocks analyzed are coherent and suggest that the age of $\mathrm{Hf} \mathrm{T}_{\mathrm{DM}}^{\mathrm{C}}$ is Neoarchean $2.8 \mathrm{Ga}$. 

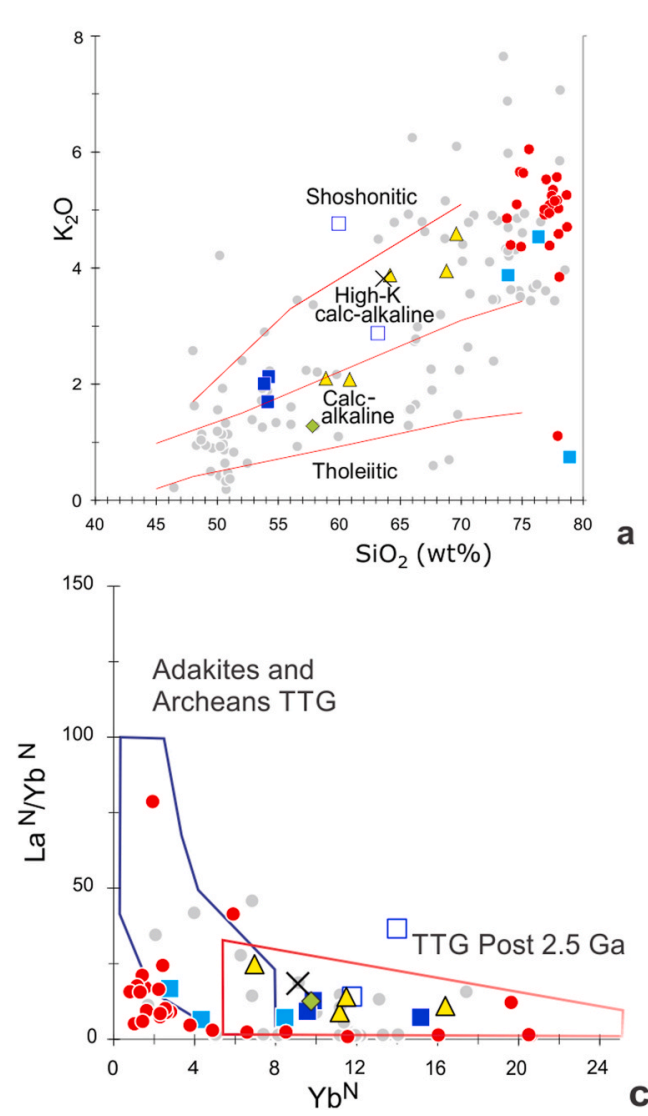
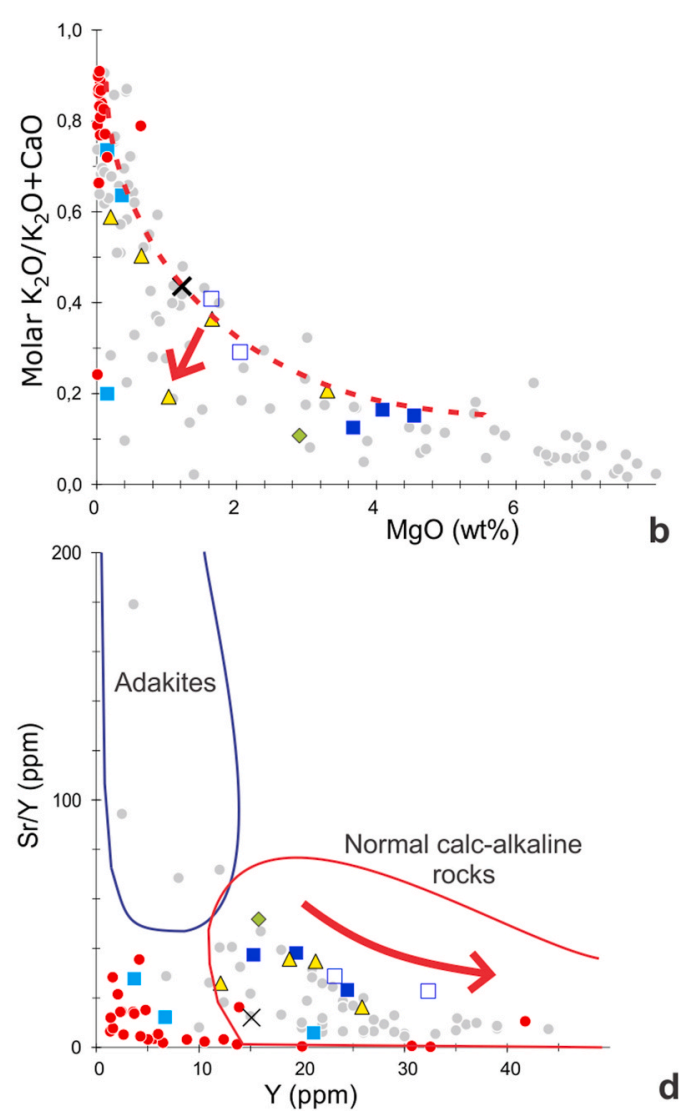

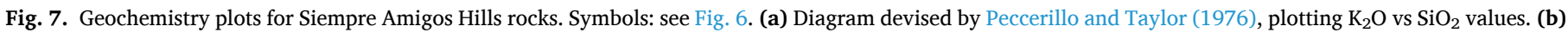

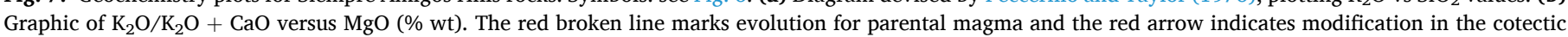

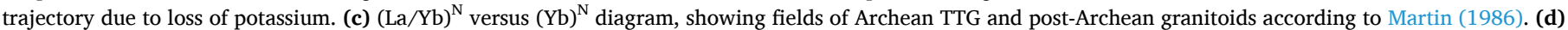
$\mathrm{Sr}$ /Y diagram with fields of adakites and Archean TTG and normal calc-alkaline rocks, from Drummond and Defant (1990).

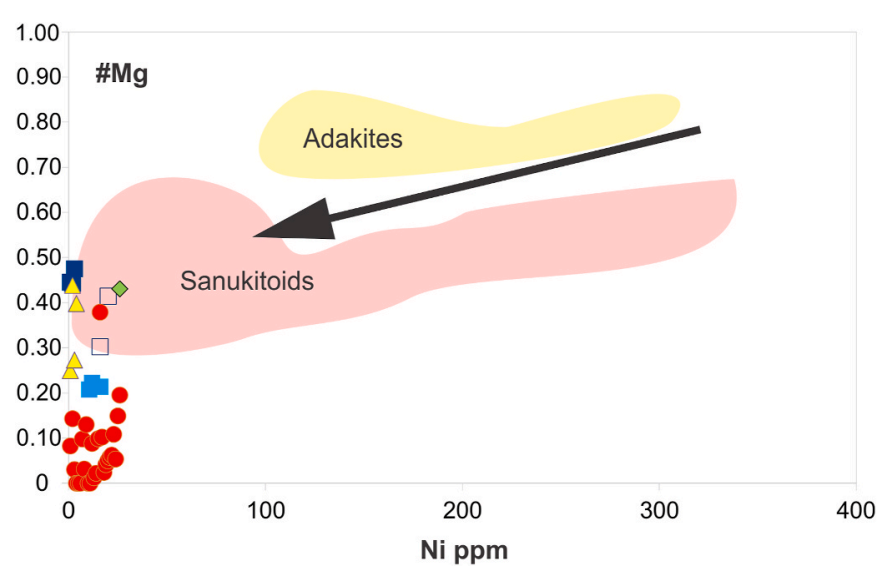

Fig. 8. Ni concentrations versus Mg number for SA rocks. In the yellow field there are values for adakites and in the pink field values from the sanukitoids and Archean shoshonites database are plotted (Stevenson et al., 1999). The $\mathrm{Mg} \#$ values are all calculated assuming $\mathrm{FeO}=$ total iron. Adakites show a much higher $\mathrm{Mg}$ content at higher levels of $\mathrm{SiO} 2$ content than SA rocks and the sanukitoid suites, and less Ni. Archean shoshonites are also quite similar to mafic compositions of the sanukitoid suites. The arrow indicates the direction of crystal fractionation of olivine-pyroxene bearing assemblages (Stevenson et al., 1999). Symbols: see Fig. 6.

\section{Discussion}

\subsection{Geochemical characterization for RPC and implications for tectonic setting}

There is evidenced for a genetic relationship between SA rocks, considering the complete data displayed in Harker diagrams (Fig. 5), showing a mafic tendency, with silica values ranging from 50 to $55 \%$, and a felsic tendency, with $65-75 \%$ silica. Classification based on REE elements (Fig. 9), shows a general evolution from andesite/basalt to rhyolite compositions, suggesting differentiation processes recorded in the Paleoproterozoic rocks as a whole. Granitic samples show a high degree of dispersion in REE and $\mathrm{Zr} / \mathrm{TiO} 2$ as compared with $\mathrm{Nb} / \mathrm{Y}$ values; this may be attributed to post-magmatic processes since REE are not totally immobile, according to Rollinson (1993). Evidence of alteration in granites is only represented by sericitization, which is intense in some feldspar crystals, being mylonitic deformation intense leading to striped gneiss microstructures. Thus, granites show a greater degree of mobilization of REE elements, probably in response to intense mylonitization and hydrothermalism. At the same time, the mobilization of REE elements in granite suggests that partial melting event may have also intervened as it is indicated by the younger crystallization ages of zircons.

Granulites seem to be the most controversial rock in terms of classification, since the mineral assemblage is typical of charnockite rocks. Many granulite facies rocks can be thoroughly described using appropriate mineral and textural criteria, while for some granulite facies rocks the specific term of charnockitic may be used (Robertson, 1999). Igneous textures are masked by intense mylonitization in SA granulite. Its dioritic or andesitic composition refers to the bulk chemical 

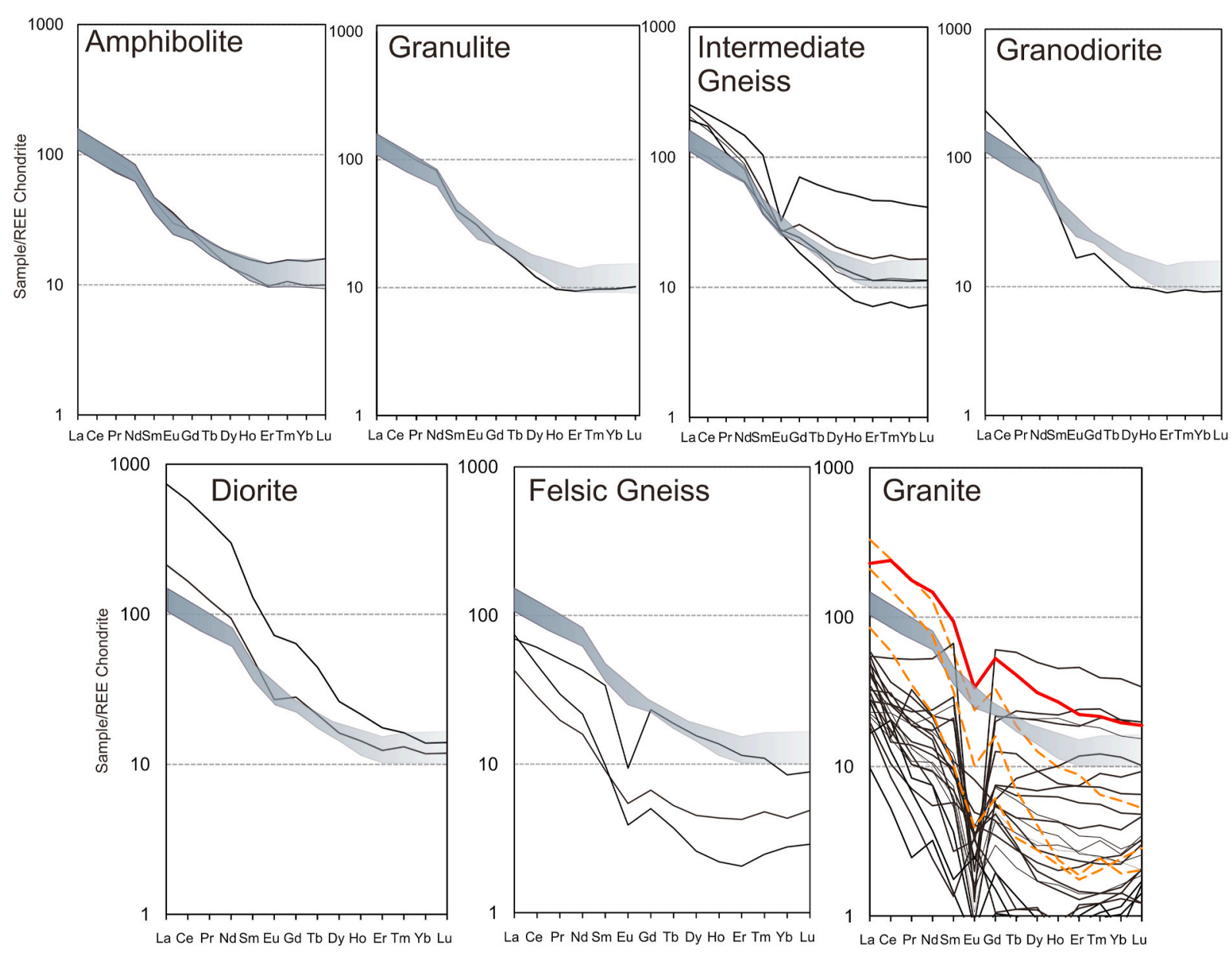

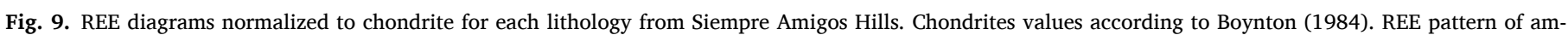

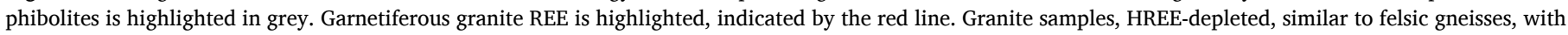
negative Eu anomalies, are shown by the orange broken lines.

composition. However, as these are actually high grade metamorphic rock, modifications in rock geochemistry by fluid-driven removal of mobile elements, and possibly of a partial melt, may have changed the bulk composition slightly (Robertson, 1999). Garnet may be primary (magmatic) or formed in the course of high-pressure metamorphic reactions and also may be present in both peraluminous and metaluminous charnockite rocks (Frost and Frost, 2008b). Garnet compositions (Angeletti et al., 2016a) are similar to garnets crystallized from granitic peraluminous magmas, as compiled by Miller and Stoddard (1981). Most of the compiled data contain more than $10 \%$ spessartine, so a metamorphic source may be suggested for SA garnets that present values below $10 \%$. Regarding orthopyroxene, this may be metamorphic from a granulite-facies event and also the relicts of this phase in the gneisses, perhaps pointing to the granulite lens as a more preserved portion of the association that was less affected by fluid-assisted solid-state recrystallization. Thus, the SA granulites protolith could be regarded as having charnockitic composition, taking into account: 1) the above-mentioned effects of mylonitization; 2) overlaps between the two charnockitic definitions; and 3) a report of similar rocks in the area of southern Tandilia by Jorgensen et al. (2008).

A remarkable point to note as regards SA bulk rock geochemistry is a slightly peraluminous trend, showing ISA $>1$, very close to the metaluminous field (Fig. 6c). Reflecting this trend, alumina-rich minerals are scarce, except for garnet. Pankhurst et al. (2003) classified granitoids in Tandilia outcrop locations northwest of SA as I-type originating due to the melting of preexisting mafic igneous rocks (Chappell and White, 1974), as a result of the abundance of $\mathrm{Hbl}$ and the absence of peraluminous minerals. At the northern and southern extremities of SA, metaluminous minerals such as $\mathrm{Hbl}$ are abundant, not only as a retrograde product in pyroxenes from felsic granulite enclaves, but also as a primary magmatic phase in gneiss since it also appears with a subhedral to euhedral shape, without any textural relationship with pyroxene. Another typical metaluminous mineral is allanite, observed as an accessory phase in almost all lithologies. Thus, SA rocks probably derive from the melting of Paleoproterozoic host igneous units due to the presence of $\mathrm{Hbl}$ and Aln, the scarcity of alumina-rich minerals and their metaluminous-to-slightly peraluminous character. The "ferroan" character of most of the rocks, except for amphibolites, as well as the degree of abnormally high oxidation, especially regarding the peraluminous granites, could be result of deformation or alteration effects. By contrast, amphibolite shows a magnesian tendency, having affinity with relatively hydrous oxidizing magmas and source regions related to a subduction setting (Frost et al., 2001). This petrological interpretation is consistent with the calc-alkaline classification observed in the diagram devised by Peccerillo and Taylor (1976) (Fig. 7a), with high K values.

In sum, most SA rocks are rich in $\mathrm{Fe}$ and $\mathrm{K}$ as a primary signature and show geochemistry consistent with a cogenetic magma suite. In Fig. $7 \mathrm{~b}$ the decrease in potassium in the direction indicated by the red arrow is interpreted as the result of the extraction of liquid; these samples should be richer in $\mathrm{SiO}_{2}$ than their equivalents along the curve, contrary to this case. Therefore, this modification in the cotectic trajectory which shows loss of potassium, which is more evident in gneiss as can be seen in the sample indicated by the red arrow in Fig. 7b, is probably caused by the circulation of metamorphic fluids, perhaps during mylonitization. At the 


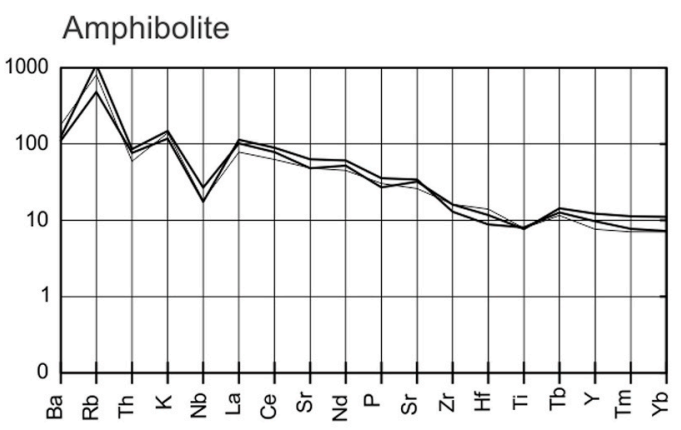

Granulite
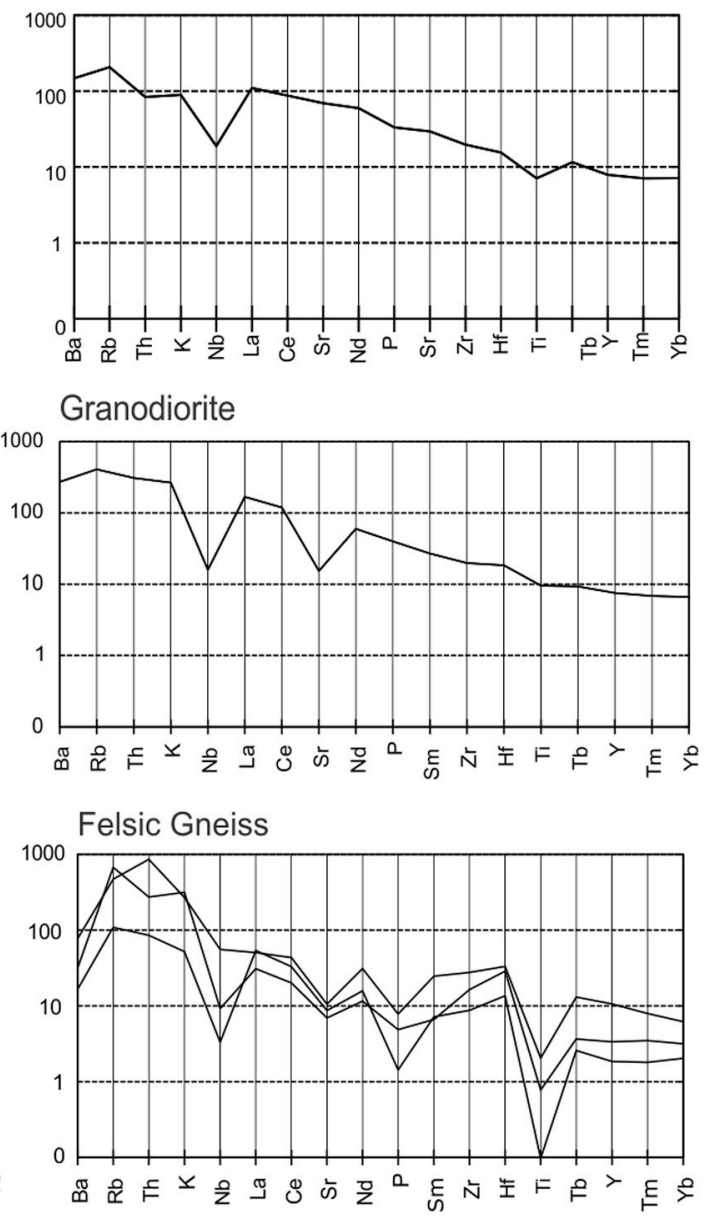

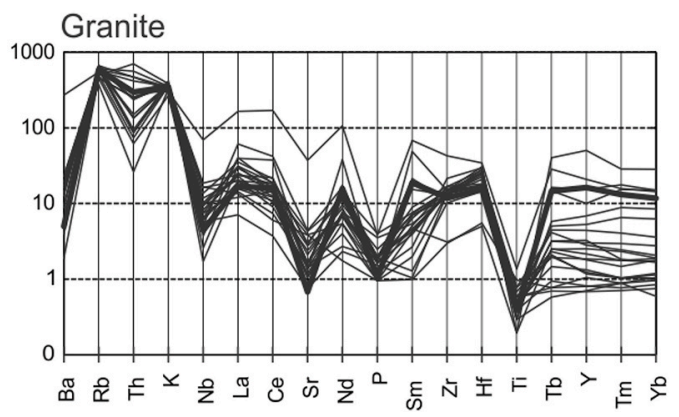

Fig. 10. Spider diagrams of incompatible elements normalized to MORB for each lithology from Siempre Amigos Hill.

same time, in the amphibolites, high $\mathrm{Ca}$ and $\mathrm{K}$ values seem to be magmatic.

\subsection{Comparison with typical Archean to Paleoproterozoic geochemical trends}

Notable aspects arising from the present study are typical patterns of subduction zones in incompatible HFSE elements and a volcanic-arc setting interpretation for SA rocks (Figs. 10-12). Granitic samples display different heterogeneous REE patterns, while only a few granite samples are HREE-depleted, similar to felsic gneisses, with negative Eu anomalies (see orange broken line in Fig. 9). A genetic relationship between SA rocks is suggested, especially it can be seen as the garnetiferous granite REE pattern is akin to an intermediate gneiss REE pattern, represented as by the continuous red line in Fig. 9. The high level of dispersion observed in the major elements could be attributed to modification through alteration or episodes of fluid circulation during deformation, but a chaotic REE pattern as found in granites could indicate a response to intense alteration or a magmatic process, such as different degrees of partial melting. Also, the LIL mobile elements group shows a contrast in its behavior, which is marked in granitic rocks, and which may be due to dispersion controlled by fluid, or the crustal contamination of magmas (Rollinson, 1993).

Comparisons between SA rocks and TTG, adakites, sanukitoids and other characteristic Archean-Paleoproterozoic granitoids were carried out in order to propose a new evolutionary model with significant genetic meaning. According to Martin and Moyen (2012), adakites and TTGs are distinguished from common granitoids and arc lavas due to their intermediate-to-acid composition, lack of mafic components, characteristic sodic composition and adakitic signature, given by high $\mathrm{Sr} / \mathrm{Y}$ and $\mathrm{La} / \mathrm{Yb}$ ratios. Besides this, adakite and TTG definitions imply $\mathrm{Nb}-\mathrm{Ta}$ negative anomalies typical of a tectonic arc setting, denoted by its 


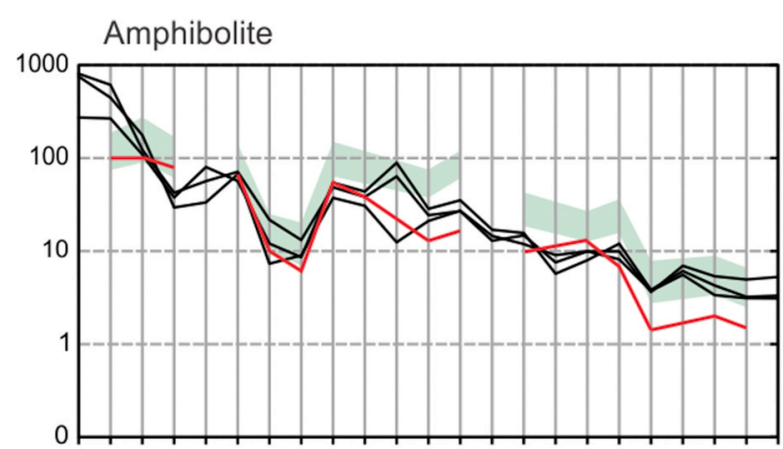

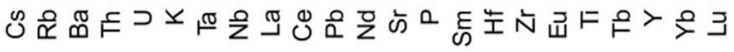
Intermediate Gneiss
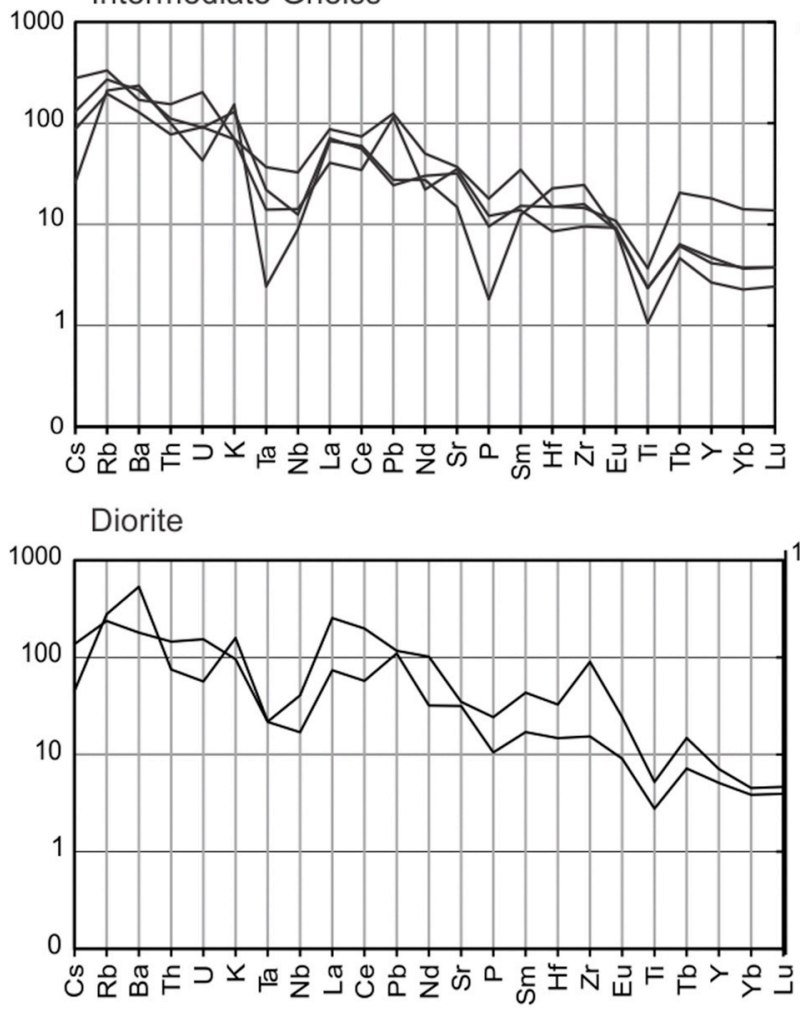

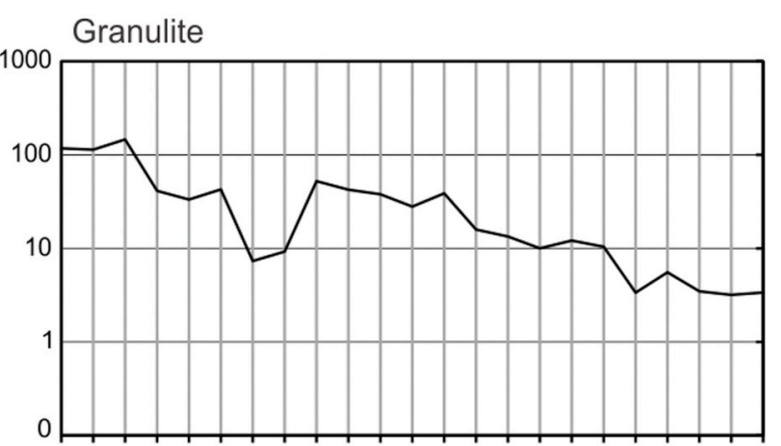

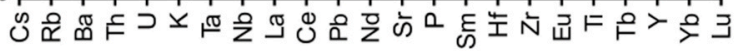

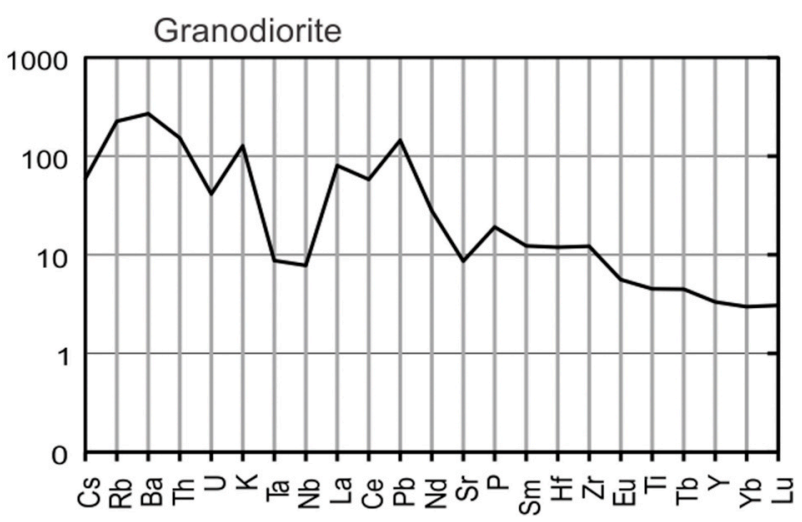

Felsic Gneiss

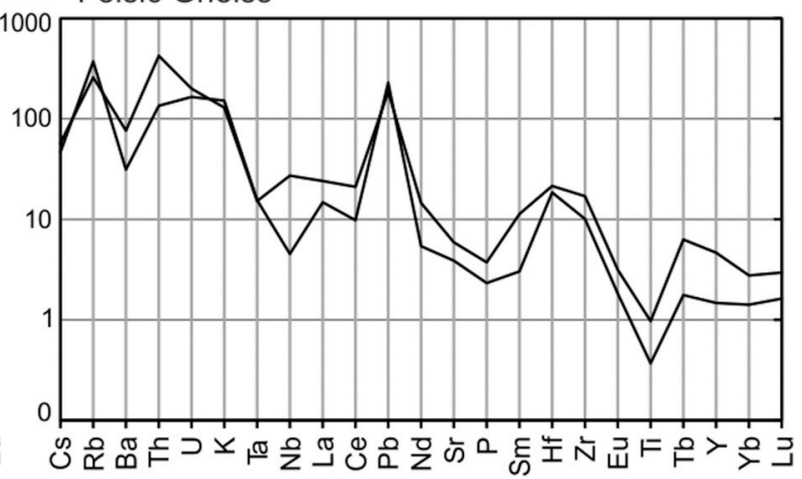

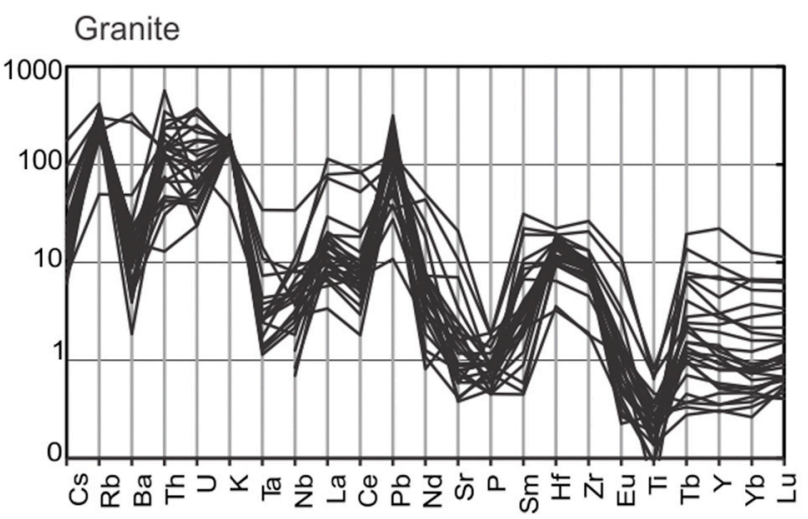

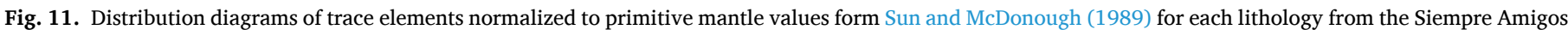

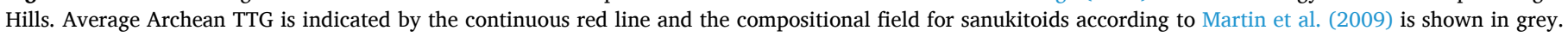

calc-alkaline nature. Compositional similarities between present-day adakites known to have formed in subduction environments and TTG, with apparent high-pressure genesis, have led to the conclusion that the latter also formed via partial melting of subducted oceanic crust slab (Martin, 1986, 1999) leaving a garnet amphibolitic or eclogitic residue
(Martin and Moyen, 2012). Considering that Archean geotherms were significantly greater in size than typical present-day geotherms (e.g. Bickle, 1986) and quite similar to present-day zones of hotter subduction which are propitious for adakite genesis, it is clear that Archean global conditions have become more exceptional since the late Archean era. In 

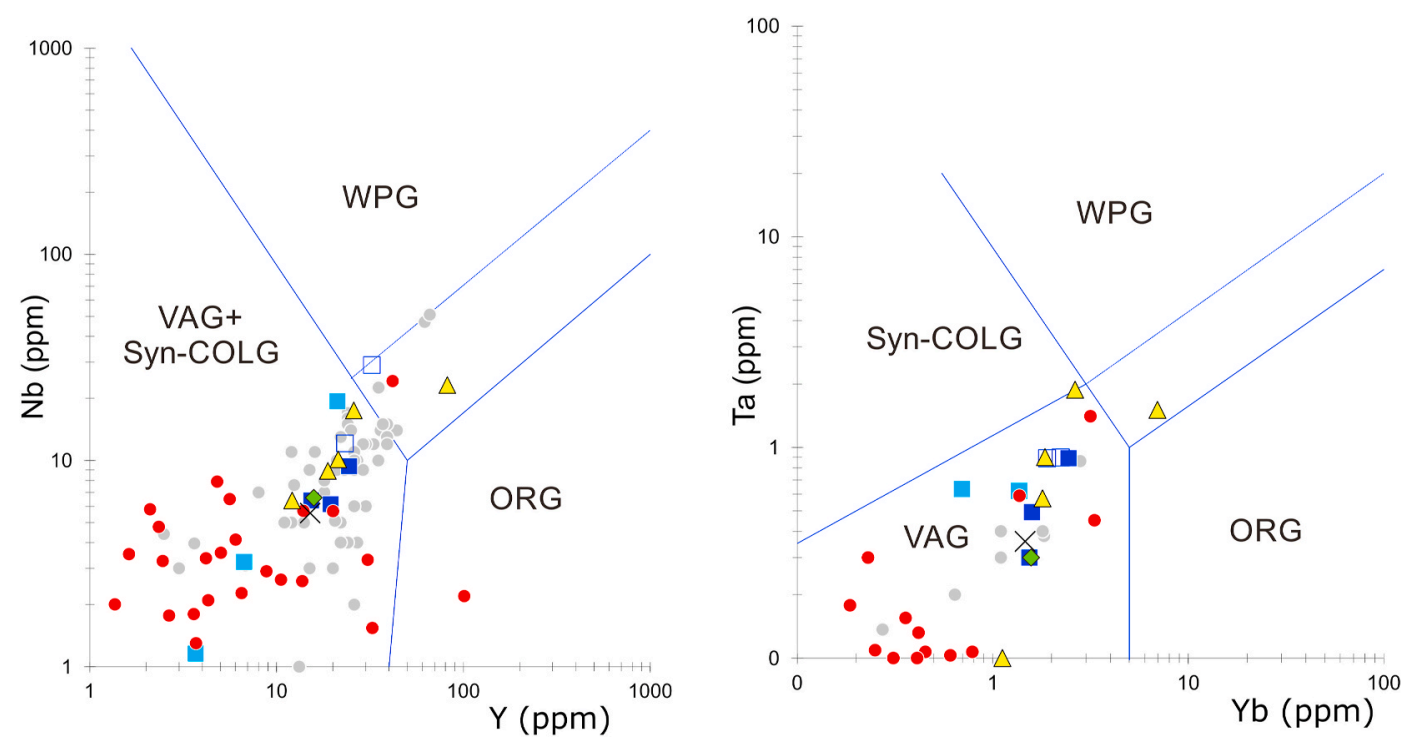

Fig. 12. Discriminant tectonic diagram, after Pearce et al. (1984). For symbols, see Fig. 6.

spite of Condie (2005) having recently challenged the idea of adakites being invariably considered as a petrogenetic analogue for all TTGs, the discussion here is based on the last assumption.

SA rocks comprise intermediate to acidic compositions, like TTGs and adakites, although they reach more mafic terms such as amphibolites. Sodic composition is a requirement for adakite rocks not satisfied by SA rocks, showing a similar trend to present-day arc rocks according to Martin and Moyen (2012) diagram, with $\mathrm{K}_{2} \mathrm{O} / \mathrm{Na}_{2} \mathrm{O}$ ratios ranging from 0.19 to 2.69. Neither is the requirement for a high $\mathrm{Sr} / \mathrm{Y}$ and $\mathrm{La} / \mathrm{Yb}$ adakitic signature fulfilled, and samples show a greater degree of similarity with those of normal calc-alkaline rocks and post-2.5 Ga TTGs. The very gradual decrease in the $\mathrm{Sr} / \mathrm{Y}$ ratio, and increase in $\mathrm{Y}$, approximates to the notion of a low-pressure differentiation process at a shallow setting with plagioclase fractionation as source. Average arc patterns in trace-element data for adakites and TTGs given by Martin and Moyen (2012) are very similar to those of SA rocks. Their relatively high $\mathrm{K}_{2} \mathrm{O}$ content ( $>2 \mathrm{wt} \%$ ) may be due to a greater extent to fractional crystallization or remelting processes, fairly common in granitoid rocks related to the Neoarchean-Paleoproterozoic transition (Martin et al., 2010). Another suite of granitoids widespread in Late Archean terranes but represented to a lesser degree than TTGs, is a group of high-Mg dioritic magmas called sanukitoids (e.g. Shirey and Hanson, 1984; Martin et al., 2005). These rocks have a dioritic-to-granodioritic composition, are rich in mafic minerals, such as $\mathrm{Bt}, \mathrm{Hbl}$ and $\mathrm{Cpx}$, with rare $\mathrm{Opx}$, and are characterized by high $\mathrm{MgO}, \mathrm{Mg} \#, \mathrm{Cr}, \mathrm{Ni}$, and $\mathrm{K}_{2} \mathrm{O}$ values. Even though $\mathrm{SA}$ rocks are rich in potassium, only a few samples of amphibolites are characterized by high concentrations of mantle elements, such as $\mathrm{Ni}$ (13.69-17.51 ppm), Cr (132.99-180.81 ppm) and V (118.99-166 ppm), as well as Ba (764.94-1232 ppm) and $\mathrm{Sr}(567.47-742.93 \mathrm{ppm})$ as crustal elements. This enrichment of crustal and mantle elements suggests that SA amphibolite is chemically akin to sanukitoids. According to Halla et al. (2009) Laurent et al. (2014) and Semprich et al. (2015) among others, based on experimental data, the two above-mentioned anomalous concentrations are the product of interactions between mantle peridotite and melts enriched in incompatible elements, such as totally-melted TTG crust.

Despite the comparisons with late Archean granitoids discussed above, most SA granitoids display a wide spectrum of $\mathrm{SiO}_{2}, \mathrm{~K}_{2} \mathrm{O}$ and $\mathrm{CaO}$ values, similar to calcic to calc-alkaline series. In addition, they represent magnesian magmas, in terms of amphibolites and some diorites and intermediate gneiss, metaluminous to slightly peraluminous at the most differentiated extremes. LILE enrichment and HFSE depletions, especially in $\mathrm{Nb}$ and $\mathrm{Ti}$, are aspects which all samples share, showing a characteristic pattern of Ti-rich phase accumulation such as high pressure melt residue, typical of subduction-related magmas. A Nb-negative anomaly is also characteristic of the continental crust and may provide an indication of crust participation in magmatic processes (Rollinson, 1993). Furthermore, they exhibit moderate-to-high REE fractionation, attributed to HREE garnet retention in the source region. Negative Eu peaks are also present in some gneiss, granodiorites, diorites and granite samples, and reflect the retention of this element by plagioclase during magma differentiation. In granites, a positive Eu peak is also observed in most samples, interpreted as resulting from a high concentration of this element in plagioclase during magma crystallization. This geochemical signature is typical of modern subduction-related tectonic settings (Foley et al., 2000; Klemme et al., 2006) where magma generation occurs due to successive episodes of mantle-wedge partial melting, which is metasomatized by fluids released from the subducted oceanic plate. Such arc-related magmas rise rapidly to the crust where they undergo fractional crystallization (e.g. Grove et al., 2003). The tectonic discriminant diagram of Pearce et al. (1984) (Fig. 12) also confirms the proposed arc-collisional tectonic setting, as with other Tandilia rocks, whereas the major element triangular diagram with fields after Laurent et al. (2014) points to an igneous high-K mafic source, with contributions from metasedimentary and tonalitic sources (Fig. 16). Thus, the source region for SA rocks seems to be markedly heterogeneous, suggesting different individual melting origins for rocks with modern-arc subduction affinities and subordinated sanukitoids, also with possible crust contamination.

Despite the fact that the SA rocks are younger than $2.1 \mathrm{Ga}$, a transition stage towards modern-arc subduction or towards favorable conditions for the genesis of sanukitoids is inferred (Martin et al., 2009). In a recent study, a late, "Archean-style" tectonic regime has been found in the Palaeoproterozoic Mineiro Belt (Brazil) using whole-rock geochemistry of its plutons coupled with zircon $\mathrm{Hf}$ and $\mathrm{O}$ isotopes from these rocks and detrital grains from the local (meta)sediments: Moreira et al. (2020) reported that the TTG-sanukitoid transition, which signals the opening of a mantle wedge, occurred in the Mineiro Belt during the early Proterozoic "magmatic lull" as the last stage prior to the plate tectonics regime.

\subsection{Regional correlation of magmatic events}

Reliable crystallization ages for rocks from the SA area were obtained for most zircons despite their displaying probable metamictization due to accumulated radiation damage and consequent isotopic disturbance. 
Table 2

U-Pb-Th SHRIMP Data on zircons from Sample 0308 (Granodiorite) and Sample 1008 (Intermediate composition gneiss). Sample 0308 (Granodiorite)

\begin{tabular}{|c|c|c|c|c|c|c|c|c|c|c|c|c|c|c|c|c|c|}
\hline \multirow[b]{2}{*}{$\begin{array}{l}\text { Grain } \\
\text { spot }\end{array}$} & \multirow[b]{2}{*}{$\begin{array}{l}\text { ppm } \\
\text { U }\end{array}$} & \multirow[b]{2}{*}{$\begin{array}{l}\text { ppm } \\
\text { Th }\end{array}$} & \multirow[b]{2}{*}{$\mathrm{Th} / \mathrm{U}$} & \multicolumn{7}{|c|}{ Isotopic Ratios } & \multicolumn{7}{|c|}{ Ages (Ma) } \\
\hline & & & & $\begin{array}{l}{ }^{207} \mathrm{~Pb} / \\
{ }^{206} \mathrm{~Pb}\end{array}$ & \pm err & $\begin{array}{l}{ }^{206} \mathrm{~Pb} / \\
{ }^{238} \mathrm{U}\end{array}$ & \pm err & $\begin{array}{l}{ }^{207} \mathrm{~Pb} / \\
{ }^{235} \mathrm{U}\end{array}$ & \pm err & Rho & $\begin{array}{l}{ }^{207} \mathrm{~Pb} / \\
{ }^{206} \mathrm{~Pb}\end{array}$ & \pm err & $\begin{array}{l}{ }^{206} \mathrm{~Pb} / \\
{ }^{238} \mathrm{U}\end{array}$ & \pm err & $\begin{array}{l}{ }^{207} \mathrm{~Pb} / \\
{ }^{235} \mathrm{U}\end{array}$ & \pm err & $\begin{array}{l}\% \\
\text { disc }\end{array}$ \\
\hline 03-1.1 & 735.4 & 100.9 & 0.14 & 0.10542 & 0.00367 & 0.16404 & 0.00607 & 2.38432 & 0.12141 & 0.73 & 1721.5 & 62.6 & 979.2 & 33.7 & 1237.9 & 37.1 & 20.8 \\
\hline $03-1.2$ & 323.0 & 98.8 & 0.31 & 0.11827 & 0.00067 & 0.22375 & 0.00268 & 3.64857 & 0.05011 & 0.87 & 1930.1 & 10.2 & 1301.7 & 14.2 & 1560.2 & 11.0 & 16.6 \\
\hline 03-10.1 & 257.4 & 119.9 & 0.48 & 0.12923 & 0.00059 & 0.37713 & 0.00191 & 6.71976 & 0.05173 & 0.66 & 2087.5 & 8.0 & 2062.8 & 8.9 & 2075.2 & 6.8 & 0.6 \\
\hline 03-11.1 & 457.5 & 133.6 & 0.30 & 0.12852 & 0.00017 & 0.33378 & 0.00157 & 5.91458 & 0.03600 & 0.77 & 2077.7 & 2.4 & 1856.7 & 7.6 & 1963.4 & 5.3 & 5.4 \\
\hline 03-12.1 & 307.4 & 125.0 & 0.42 & 0.12743 & 0.00051 & 0.34598 & 0.00046 & 6.07895 & 0.03361 & 0.24 & 2062.9 & 7.0 & 1915.4 & 2.2 & 1987.2 & 4.8 & 3.6 \\
\hline 03-13.1 & 750.2 & 121.8 & 0.17 & 0.13392 & 0.00139 & 0.16371 & 0.00197 & 3.02278 & 0.04920 & 0.74 & 2149.9 & 18.0 & 977.4 & 10.9 & 1413.4 & 12.5 & 30.8 \\
\hline 03-13.2 & 348.2 & 166.6 & 0.49 & 0.13044 & 0.00031 & 0.28193 & 0.00257 & 5.07048 & 0.05108 & 0.9 & 2103.9 & 4.0 & 1601.1 & 12.9 & 1831.2 & 8.6 & 12.6 \\
\hline 03-14.1 & 214.8 & 120.2 & 0.57 & 0.13371 & 0.00775 & 0.29775 & 0.02194 & 5.48914 & 0.51490 & 0.79 & 2147.3 & 97.8 & 1680.1 & 109.9 & 1898.9 & 83.9 & 11.6 \\
\hline 03-15.1 & 472.2 & 344.1 & 0.75 & 0.19338 & 0.00177 & 0.32251 & 0.00485 & 8.59910 & 0.15445 & 0.84 & 2771.1 & 15.0 & 1802.0 & 23.7 & 2296.5 & 16.5 & 21.6 \\
\hline 03-16.1 & 303.3 & 247.6 & 0.84 & 0.12998 & 0.00059 & 0.22564 & 0.00164 & 4.04389 & 0.03753 & 0.78 & 2097.7 & 8.0 & 1311.6 & 8.6 & 1643.1 & 7.6 & 20.2 \\
\hline 03-17.1 & 295.9 & 120.5 & 0.42 & 0.13030 & 0.00070 & 0.34445 & 0.00330 & 6.18847 & 0.07144 & 0.83 & 2102.1 & 9.4 & 1908.0 & 15.8 & 2002.8 & 10.1 & 4.8 \\
\hline 03-18.1 & 165.9 & 83.1 & 0.51 & 0.13630 & 0.00071 & 0.36012 & 0.00255 & 6.76801 & 0.06423 & 0.75 & 2180.7 & 9.0 & 1982.8 & 12.1 & 2081.5 & 8.4 & 4.8 \\
\hline 03-19.1 & 108.4 & 54.4 & 0.51 & 0.13037 & 0.00064 & 0.38065 & 0.00296 & 6.84222 & 0.06758 & 0.79 & 2102.9 & 8.6 & 2079.3 & 13.8 & 2091.2 & 8.8 & 0.6 \\
\hline 03-2.1 & 266.9 & 97.6 & 0.38 & 0.13075 & 0.00069 & 0.36214 & 0.00695 & 6.52871 & 0.13203 & 0.95 & 2108.1 & 9.2 & 1992.3 & 32.9 & 2049.8 & 18.0 & 2.8 \\
\hline 03-2.2 & 512.2 & 409.4 & 0.82 & 0.13334 & 0.00056 & 0.18959 & 0.00161 & 3.48556 & 0.03544 & 0.84 & 2142.5 & 7.4 & 1119.1 & 8.7 & 1524.0 & 8.1 & 26.6 \\
\hline 03-20.1 & 320.7 & 130.9 & 0.42 & 0.12760 & 0.00045 & 0.35674 & 0.00361 & 6.27635 & 0.07100 & 0.89 & 2065.1 & 6.4 & 1966.7 & 17.1 & 2015.2 & 10.0 & 2.4 \\
\hline $03-20.2$ & 199.2 & 119.4 & 0.61 & 0.13163 & 0.00123 & 0.36835 & 0.00539 & 6.68555 & 0.11850 & 0.83 & 2119.9 & 16.2 & 2021.6 & 25.4 & 2070.7 & 15.8 & 2.4 \\
\hline 03-21.1 & 839.1 & 162.0 & 0.20 & 0.12075 & 0.00092 & 0.12117 & 0.00127 & 2.01746 & 0.02715 & 0.78 & 1967.3 & 13.6 & 737.3 & 7.3 & 1121.4 & 9.2 & 34.2 \\
\hline 03-21.2 & 103.6 & 69.2 & 0.69 & 0.13096 & 0.00062 & 0.35145 & 0.00203 & 6.34624 & 0.05249 & 0.7 & 2110.9 & 8.2 & 1941.5 & 9.7 & 2024.9 & 7.3 & 4.2 \\
\hline 03-22.1 & 297.7 & 205.8 & 0.71 & 0.13171 & 0.00448 & 0.33649 & 0.02138 & 6.11083 & 0.44087 & 0.88 & 2120.9 & 58.4 & 1869.7 & 103.9 & 1991.8 & 65.0 & 6.2 \\
\hline 03-23.1 & 112.6 & 35.1 & 0.32 & 0.12675 & 0.00048 & 0.36926 & 0.00499 & 6.45307 & 0.09355 & 0.93 & 2053.3 & 6.6 & 2025.9 & 23.5 & 2039.5 & 12.8 & 0.6 \\
\hline 03-24.1 & 226.5 & 76.4 & 0.35 & 0.13174 & 0.00057 & 0.37015 & 0.00587 & 6.72335 & 0.11319 & 0.94 & 2121.3 & 7.6 & 2030.1 & 27.7 & 2075.7 & 15.0 & 2.2 \\
\hline 03-25.1 & 164.1 & 107.2 & 0.67 & 0.12808 & 0.00193 & 0.33623 & 0.00158 & 5.93783 & 0.09592 & 0.29 & 2071.9 & 26.2 & 1868.5 & 7.6 & 1966.8 & 14.2 & 5.0 \\
\hline 03-26.1 & 765.4 & 333.6 & 0.45 & 0.09852 & 0.00045 & 0.15350 & 0.00147 & 2.08525 & 0.02344 & 0.85 & 1596.3 & 8.6 & 920.6 & 8.3 & 1144.0 & 7.8 & 19.6 \\
\hline 03-27.1 & 1235.8 & 847.5 & 0.70 & 0.09492 & 0.00107 & 0.12421 & 0.00046 & 1.62564 & 0.02020 & 0.3 & 1526.5 & 21.2 & 754.7 & 2.6 & 980.2 & 7.9 & 23.0 \\
\hline 03-28.1 & 47.9 & 19.3 & 0.41 & 0.12477 & 0.00337 & 0.26870 & 0.00547 & 4.62264 & 0.15717 & 0.6 & 2025.5 & 47.2 & 1534.2 & 27.8 & 1753.4 & 28.8 & 12.6 \\
\hline 03-29.1 & 314.9 & 172.4 & 0.56 & 0.12392 & 0.00058 & 0.29260 & 0.00242 & 4.99957 & 0.05076 & 0.81 & 2013.5 & 8.2 & 1654.5 & 12.1 & 1819.2 & 8.6 & 9.0 \\
\hline 03-3.1 & 170.1 & 92.2 & 0.56 & 0.13105 & 0.00041 & 0.37671 & 0.00332 & 6.80686 & 0.06823 & 0.88 & 2112.1 & 5.4 & 2060.9 & 15.6 & 2086.6 & 8.9 & 1.2 \\
\hline 03-30.1 & 610.5 & 104.5 & 0.18 & 0.11337 & 0.00077 & 0.23029 & 0.00163 & 3.59975 & 0.03761 & 0.68 & 1854.1 & 12.2 & 1336.0 & 8.5 & 1549.5 & 8.4 & 13.8 \\
\hline 03-31.1 & 180.6 & 91.0 & 0.52 & 0.13012 & 0.00063 & 0.35528 & 0.00430 & 6.37426 & 0.08632 & 0.89 & 2099.7 & 8.4 & 1959.8 & 20.5 & 2028.7 & 11.9 & 3.4 \\
\hline 03-32.1 & 204.4 & 104.4 & 0.52 & 0.12984 & 0.00087 & 0.36952 & 0.00216 & 6.61552 & 0.06354 & 0.61 & 2095.9 & 11.8 & 2027.1 & 10.1 & 2061.4 & 8.5 & 1.6 \\
\hline 03-33.1 & 193.1 & 88.9 & 0.47 & 0.12999 & 0.00113 & 0.36691 & 0.00681 & 6.57604 & 0.13676 & 0.89 & 2097.9 & 15.0 & 2014.8 & 32.2 & 2056.1 & 18.5 & 2.0 \\
\hline $03-4.1$ & 420.3 & 211.1 & 0.52 & 0.13200 & 0.00046 & 0.31481 & 0.00194 & 5.72948 & 0.04550 & 0.78 & 2124.7 & 6.0 & 1764.3 & 9.5 & 1935.8 & 6.9 & 8.8 \\
\hline 03-5.1 & 141.5 & 69.5 & 0.50 & 0.13615 & 0.00081 & 0.37757 & 0.00195 & 7.08825 & 0.06137 & 0.6 & 2178.9 & 10.2 & 2064.9 & 9.1 & 2122.6 & 7.8 & 2.8 \\
\hline $03-6.1$ & 307.4 & 126.6 & 0.42 & 0.13287 & 0.00039 & 0.34349 & 0.00244 & 6.29295 & 0.05330 & 0.84 & 2136.3 & 5.0 & 1903.4 & 11.7 & 2017.5 & 7.5 & 5.6 \\
\hline 03-7.1 & 117.5 & 49.6 & 0.43 & 0.12971 & 0.00047 & 0.37761 & 0.00220 & 6.75359 & 0.05236 & 0.75 & 2094.1 & 6.4 & 2065.1 & 10.3 & 2079.7 & 6.9 & 0.8 \\
\hline 03-8.1 & 385.5 & 134.9 & 0.36 & 0.12747 & 0.00055 & 0.28449 & 0.00168 & 5.00001 & 0.04065 & 0.72 & 2063.3 & 7.6 & 1613.9 & 8.4 & 1819.3 & 6.9 & 11.2 \\
\hline $03-8.2$ & 796.2 & 365.4 & 0.47 & 0.11823 & 0.00075 & 0.16060 & 0.00161 & 2.61816 & 0.03249 & 0.81 & 1929.7 & 11.2 & 960.1 & 8.9 & 1305.7 & 9.1 & 26.4 \\
\hline 03-9.1 & 553.6 & 200.3 & 0.37 & 0.12767 & 0.00039 & 0.31884 & 0.00271 & 5.61260 & 0.05459 & 0.88 & 2066.1 & 5.4 & 1784.0 & 13.2 & 1918.0 & 8.4 & 7.0 \\
\hline \multicolumn{18}{|c|}{ Sample 1008 (Intermediate composition gneiss) } \\
\hline & & & & \multicolumn{7}{|c|}{ Isotopic Ratios } & \multicolumn{7}{|c|}{ Ages (Ma) } \\
\hline $\begin{array}{l}\text { Grain } \\
\text { spot }\end{array}$ & $\begin{array}{l}\text { ppm } \\
\mathrm{U}\end{array}$ & $\begin{array}{l}\text { ppm } \\
\text { Th }\end{array}$ & $\mathrm{Th} / \mathrm{U}$ & $\begin{array}{l}{ }^{207} \mathrm{~Pb} / \\
{ }^{206} \mathrm{~Pb}\end{array}$ & \pm err & $\begin{array}{l}{ }^{206} \mathrm{~Pb} / \\
{ }^{238} \mathrm{U}\end{array}$ & \pm err & $\begin{array}{l}{ }^{207} \mathrm{~Pb} / \\
{ }^{235} \mathrm{U}\end{array}$ & \pm err & Rho & $\begin{array}{l}{ }^{207} \mathrm{~Pb} / \\
{ }^{206} \mathrm{~Pb}\end{array}$ & \pm err & $\begin{array}{l}{ }^{206} \mathrm{~Pb} / \\
{ }^{238} \mathrm{U}\end{array}$ & \pm err & $\begin{array}{l}{ }^{207} \mathrm{~Pb} / \\
{ }^{235} \mathrm{U}\end{array}$ & \pm err & $\begin{array}{l}\% \\
\text { disc }\end{array}$ \\
\hline $10-1.1$ & 201.1 & 63.2 & 0.32 & 0.12896 & 0.00229 & 0.39621 & 0.00182 & 7.04506 & 0.13183 & 0.25 & 2083.9 & 30.8 & 2151.6 & 8.4 & 2117.1 & 16.7 & -1.6 \\
\hline $10-10.1$ & 242.5 & 54.7 & 0.23 & 0.13053 & 0.00022 & 0.35843 & 0.00301 & 6.45099 & 0.05993 & 0.9 & 2105.1 & 3.0 & 1974.7 & 14.3 & 2039.2 & 8.2 & 3.2 \\
\hline $10-11.1$ & 82.7 & 29.3 & 0.36 & 0.13175 & 0.00044 & 0.38875 & 0.00258 & 7.06206 & 0.05833 & 0.8 & 2121.5 & 5.8 & 2117.0 & 12.0 & 2119.3 & 7.4 & 0.2 \\
\hline $10-12.1$ & 620.6 & 360.9 & 0.60 & 0.10231 & 0.00271 & 0.18235 & 0.01712 & 2.57227 & 0.25113 & 0.96 & 1666.5 & 48.0 & 1079.8 & 94.1 & 1292.8 & 74.0 & 16.4 \\
\hline 10-13.1 & 125.7 & 60.6 & 0.49 & 0.13009 & 0.01005 & 0.40880 & 0.05645 & 7.33274 & 1.16037 & 0.87 & 2099.3 & 129.6 & 2209.5 & 263.6 & 2152.8 & 152.3 & -2.6 \\
\hline
\end{tabular}


Calculated crystallization ages are $2092 \pm 12$ Ma for granodiorite and $2104 \pm 7.6$ Ma for intermediate composition gneiss. Magmatic origin is suggested by the predominance of prismatic forms, the oscillatory zoning of zircons, and $\mathrm{Th} / \mathrm{U}>0.1$, consistent with precipitation from the melt phase of felsic to intermediate composition magma (e.g. Hoskin and Schaltegger, 2003). These Rhyacian magmatic ages are consistent with those obtained by Cingolani et al. (2002) and Hartmann et al. (2002), using U-Pb SHRIMP isotopic techniques on zircons of granitoids from different areas of Tandilia. Also, Pankhurst et al. (2003) obtained $\mathrm{Sm}-\mathrm{Nd}$ isochron ages of $2140 \pm 88 \mathrm{Ma}$ for the emplacement of the igneous protolith of the western granitoids of Tandilia, further suggesting that most rocks were derived from cogenetic igneous protoliths. Granodiorite and intermediate composition gneiss zircons show external morphological similarities suggesting that they probably form a single population with a common igneous origin. Despite this, granodiorite zircons show weak oscillatory zoning, attributed to magmatic origin. The appearance of two growth stages, suggests an initial stage of zircon crystallization, a transient period of dissolution/resorption, and a final stage of zircon crystallization with a relatively high $\mathrm{U}$ content. In zircons from intermediate composition gneiss, the range in $\mathrm{Th} / \mathrm{U}$ values and the presence of banded zoned zircons together with concentric zoned zircons suggest precipitation from the melt phase with a mixture of intermediate and felsic magmas. Also, a sector-zoning pattern in zircons could be due to fractional crystallization in a granitic magma since partial melting is likely to preserve an inherited pattern.

SA rocks yield both positive and negative $\mathrm{eHf}$ values, suggesting protoliths predominantly derived from juvenile origins, with the participation of old crust recycling, and present Neoarchaean to Paleoproterozoic model ages (Fig. 15). In the case of the gneiss sample, a majority of negative values suggests the radical reworking of crust, whereas for the granodiorite sample, high $\varepsilon \mathrm{Hf}_{(\mathrm{t})}$ values which are mainly positive are interpreted as signifying the great contribution of juvenile material.

The results of in situ $\mathrm{Hf}$ isotope analysis show scarce ${ }^{176} \mathrm{Hf} /{ }^{177} \mathrm{Hf}$ initial variation ratios, ranging from 0.28127 to 0.28163 , suggesting magmatic origin of zircon grains. Lu-Hf isotopic analyses are comparable with those carried out by Cingolani (2011), who attributed the overwhelming predominance of positive $\varepsilon \mathrm{Hf}(\mathrm{t})$ values to juvenile material derivation, an alternative interpretation by these authors positing juvenile (2.27 Ga?) and crustal (older than $2.65 \mathrm{Ga}$ ) material mixture as magmatic components. $\mathrm{T}_{\mathrm{DM}}$ and $\mathrm{T}_{\mathrm{DM}}^{\mathrm{C}}$ ages are also comparable, in addition to Sm-Nd data published by Hartmann et al. (2002) and Pankhurst et al. (2003), who interpreted Neoarchean $\mathrm{T}_{\mathrm{DM}}$ ages (c.2.6 Ga). Cingolani (2011) also state that these findings agree with those for Piedra Alta, RPC outcrops in Uruguay, indicating a relatively short life for Paleoproterozoic orogeny. This contrasts with the evolution of the neighboring Nico Pérez Terrane in Uruguay, which is characterized by: 1) Archean Sm-Nd model ages (Oyhantçabal et al., 2011a); 2) exclusive negative $\varepsilon \mathrm{Hf}{ }_{(\mathrm{t})}$ values and $\mathrm{Hf} \mathrm{T}_{\mathrm{DM}}$ distribution revealing Archean episodic crustal growth with the main peaks of crustal generation occurring during the Paleo and Mesoarchean, and crustal reworking during the Proterozoic (Oriolo et al., 2016). These differences support the idea that the RPC boundary is located in the Sarandí del Yi shear zone, considering Nico Pérez Terrane as an allochthonous terrane. Moreover, new Lu-Hf data provided in the present study, coincidental with known $\mathrm{T}_{\mathrm{DM}}$ and $\varepsilon \mathrm{Hf} \mathrm{ft}_{(\mathrm{t})}$ for Tandilia terrane, provide some support for different mantle extraction times for Tandilia and Piedra Alta terranes, regarded as being distinct terranes (Bossi and Cingolani, 2009; Abre et al., 2014; Pamoukaghlian et al., 2017). The most convincing arguments in favor of this configuration are that no Archean model ages have yet been determined for Piedra Alta Terrane samples, suggesting that the terrane was formed rapidly from a juvenile source, in contrast with the protracted history suggested for the Tandilia Terrane. Whatever the case, and emphasizing similarities instead of differences, Oyhantçabal et al. (2018) put forward the reinterpretation that similar isotope data from samples taken in all areas of the RPC could indicate that the 


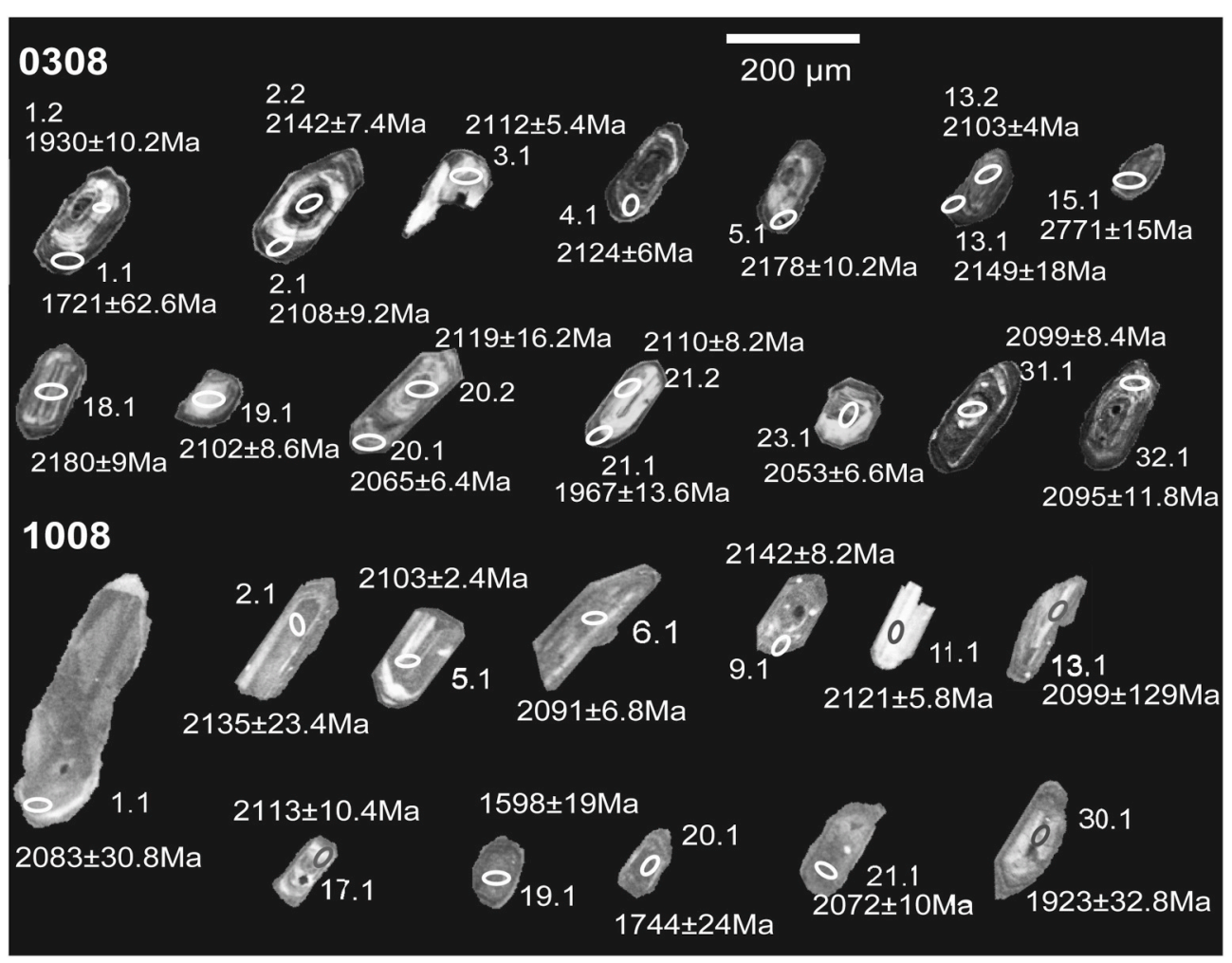

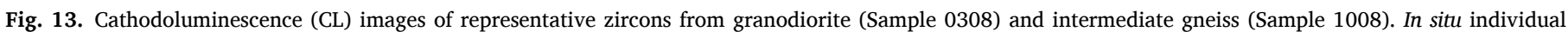
${ }^{207} \mathrm{~Pb} /{ }^{206} \mathrm{~Pb}$ ages are indicated. The spot of previous U-Pb SHRIMP analysis in the selected zircons was taken as a reference for Hafnium isotope measurement.

terranes involved are para-autochthonous, and in case of the sutures proposed this was confirmed.

Based on the analogous isotopic signature of SA granodiorite and gneiss it is assumed that a single magmatic event gave rise to these rocks, whose ages of crystallization range between 2.1 and $2 \mathrm{Ga}$, and have an average of $2.5 \mathrm{Ga} \mathrm{T}_{\mathrm{DM}}$ age for new crust extraction from the mantle. This Trans-Amazonian magmatic event is predominantly juvenile, followed by crustal recycling. Inheritance is recorded in a single core of Neoarchaean zircon, a granodiorite sample (spot $15.1=2771.1 \pm 15 \mathrm{Ma}$ ) bearing some similarity to the calculated $\mathrm{HfT}_{\mathrm{DM}}$ model age of $2.76 \mathrm{Ga}$, and in agreement with Cingolani et al. (2010), who found similar evidence in a single zircon. This inherited zircon from SA granodiorite has a distinct isotopic signature, indicated by its positive high value (16.88) calculated for $\varepsilon \mathrm{Hf}$, suggesting juvenile sources. Based on this observation, the relative subsaturation of zircons in the Paleoproterozoic of Tandilia may be inferred, while granitoids from SA and Tandilia may be classified as hot inheritance-poor granitoids with $\mathrm{TZr}>800{ }^{\circ} \mathrm{C}$ (Watson and Harrison, 1983; Miller et al., 2003). An alternative interpretation is that the source of these rocks may be rock which is poor in zircons, such as mafic rock. Therefore, the participation of magma whose source is a juvenile, slightly differentiated, igneous crust without sediment, whose crystallization age is $2.7 \mathrm{GA}$, within the range of the calculated $\mathrm{T}_{\mathrm{DM}}$ age, may be inferred. Considering that no outcrops of such Archean ages have been found in the RPC so far, these data are useful for previous proto-continent characterization. This interpretation supports the petrogenetic model of TTGs described by Laurent et al. (2014) consistent with the major element geochemistry of Southern African Pietersburg block TTG samples, with a geological history characterized by a first magmatic stage of long-lasting, intense juvenile crust formation and a second stage of recycling of this pre-existing crust. These authors proposed that new crust was formed during first magmatic stage that includes two successive steps: initial melting of the mantle to produce tholeiitic mafic rocks; and the subsequent melting of these rocks, giving rise to TTGs. The first magmatic stage is characterized by positive $\varepsilon \mathrm{Hf}$ values in zircons from TTGs. Although the age of $2.7 \mathrm{Ga}$ is slightly younger than that of those proposed by Laurent et al. (2014), the absolute lengths of the stages indicated varies considerably from one region to another. A similar model was proposed by Montefalco de Lira Santos et al. (2017) for the emplacement of the high-Al low-REE TTG Riacho das Lajes Suite which is the first record of Neoarchean rocks within the Alto Moxoto Terrane, Borborema Province, NE Brazil. The rock association mentioned was generated in a garnet-rich and fluid-absent source, which points to slab melting of oceanic crust metamorphosed under eclogite facies conditions. Nd isotopes clearly indicate a juvenile source with only minor crustal contamination. This event is interpreted as the result of subduction beneath a thick oceanic plateau or protocrust. Accretion of Rhyacian (ca. $2.1 \mathrm{Ga}$ ) magmatic arc, coeval with slab breakoff, was interpreted using data from Floresta Suites, the other basement metagranitic suite within the Alto Moxoto Terrane (Montefalco de Lira Santos et al., 2017). Hence arc magmatism was widespread in most Paleoproterozoic cratonic blocks, including the São Francisco-Congo Craton, within a context of Trans-Amazonian-Eburnean orogeny (Montefalco de Lira Santos et al., 2017). Thus, the tectonic scenario inferred for RPC using SA data is comparable to the Floresta Suites interpretation, and also consistent with the Atlantica supercontinent configuration for Paleoproterozoic Rhyacian times. Also, in São Francisco, Brazil, two episodes of TTG and sanukitoid magmatism were recently reported, one during the Neoarchean in the Piedade Complex and another during the Rhyacian in the Mantiqueira Complex, indicating that the onset of subduction-related melting of metasomatized mantle was not restricted to Neoarchaean times, but persisted into the Paleoproterozoic (Bruno et al., 2020).

5.4. Alternative tectonic evolution for Tandilia and the RPC: approaches for a petrogenetic model using new geochemical and geochronological information

The new petrogenetic model for Tandilia and the RPC based on the 

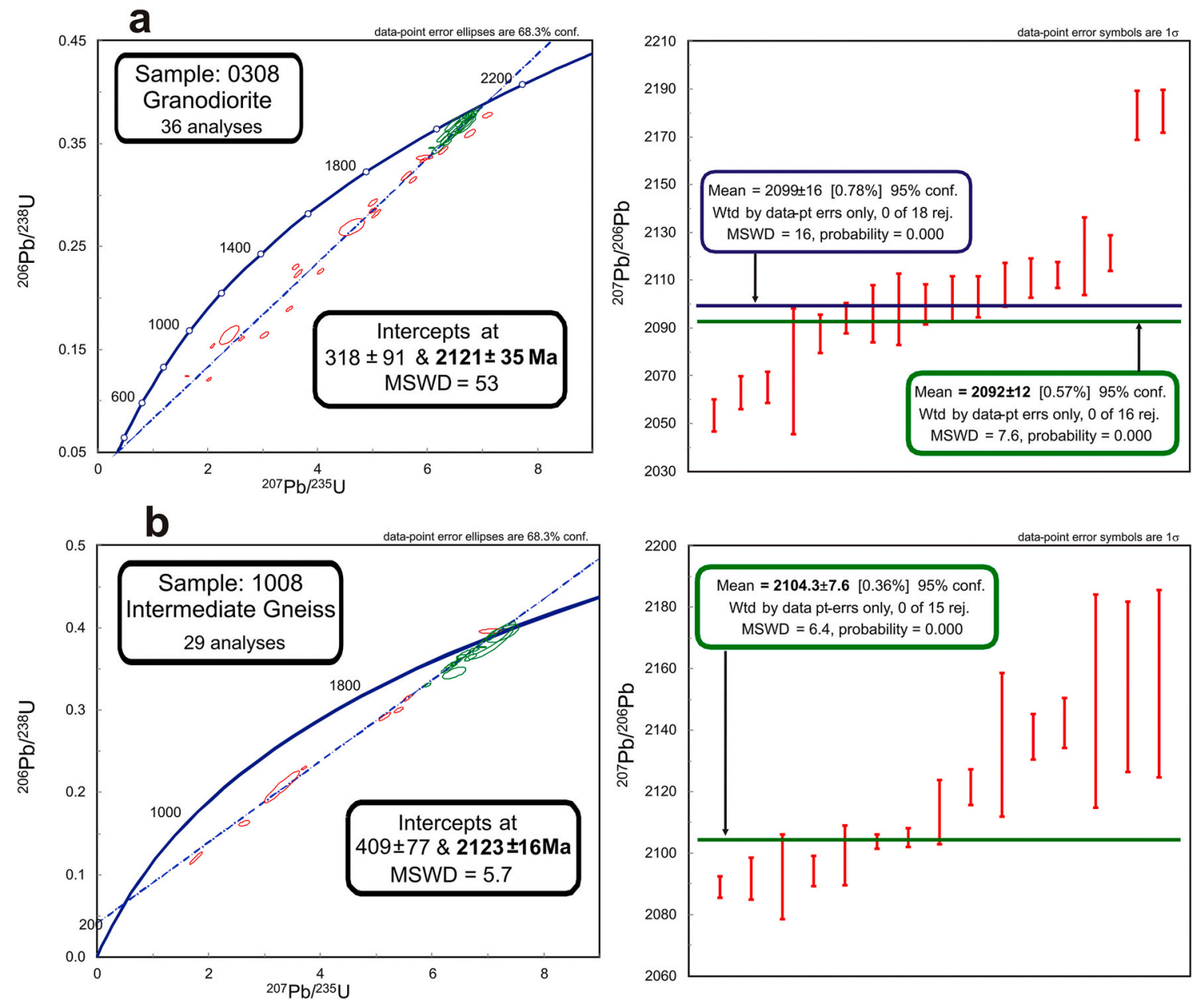

Fig. 14. Concordia- and weighted-mean U-Pb diagrams for (a) granodiorite (Sample 0308); (b) intermediate gneiss (Sample 1008). Green ellipses in U-Pb concordia diagram represent analyses used for weighted-mean age calculations.

interpretation of geochemistry and isotopic data presented in this paper points to subduction since calc-alkaline magmatism compatible with a modern-arc setting is present in Tandilia Terrane, involving thickened crust, similar to the model proposed by Chernicoff et al. (2015) and Cingolani (2011). Subduction with slab breakoff provides an explanation for the heat required to melt part of the slab and create a geochemical mantelic and crustal sanukitoid signature in SA amphibolites. As $\mathrm{TiO}_{2} \%$ is low in these sanukitoid rocks (Fig. 5), slab melts may have been greatly contaminated by the assimilation of mantle peridotite, without being totally consumed (Martin et al., 2009). SA amphibolites geochemical data accompanied by tested samples of well-documented sanukitoids and TTG suites samples compared in a Ni versus $\mathrm{Mg}$ \# diagram (Stevenson et al., 1999. Fig. 8) evidenced affinity of SA amphibolites, and also diorites, and some intermediate gneiss and granulites, with sanukitoids, the arrow indicating the direction of crystal fractionation of olivine-pyroxene bearing assemblages. Trace-element spider diagrams correlate similar SA amphibolites patterns against the sanukitoid pattern, according to Martin et al. (2009), supporting affinity with low-Ti types. Thus, this is the first paper to put forward the hypothesis of a sanukitoid precursor for RPC Rhyacian magmatism. As a result, the key element for correlation from a petrological and geochronological point of view may be regarded as the presence of sanikutoids.

Concerning regional geology, the common structure in the rocks of the SA Hills provides a parallel to lenticular banding. Such banding shows interspersing under plastic conditions between slightly deformed granitoids and a range of medium to high-grade metamorphic rocks as partially melted felsic and intermediate composition gneisses, granulites and amphibolites. This region thus presents magmatic arc low-deformed calc-alkaline granitoids as granodiorite $(2092 \pm 12 \mathrm{Ma})$ intruding into host rocks of medium to high metamorphic grade including gneiss $(2104 \pm 7.6 \mathrm{Ma})$ and amphibolite with a sanukitoid signature, except for the localized low-temperature shear zones that affected the whole sequence. All these host rocks could eventually be associated with the same P-T-t path, elucidated by EMP geochronology on monazite, as was shown in Cinco Cerros, Balcarce (Massonne et al., 2012). The model proposed for Cinco Cerros is based on initial metamorphic conditions of $6.7 \mathrm{kbar}$ and $670^{\circ} \mathrm{C}$ and final conditions of $4.5 \mathrm{kbar}$ and $600{ }^{\circ} \mathrm{C}$. This P-T evolution and timing, as well as the overall geological circumstances of the exposed RPC in the Tandilia belt were explained by Massonne et al. (2012) on the basis of a heating event resulting from magmas that intruded during the Trans-Amazonian cycle $(2073 \pm 11.4 \mathrm{Ma})$, followed by slow exhumation $(1913 \pm 11.0 \mathrm{Ma})$ and thermal relaxation (1805 \pm $20.8 \mathrm{Ma}$ ). Thus, metamorphism conditions in SA are relatively medium pressure/temperature, and although the "Barrovian" style may be debatable as holding in those times, this could provide further evidence of the beginning of 'modern subduction' and the slowing down of the geodynamic process at the onset of the Proterozoic. This means metamorphic conditions should result from Paleoproterozoic subduction as well as the syn-to late-metamorphic intrusion of huge volumes of Rhyacian calc-alkaline magmatism, represented in this study by granodiorite and granites.

Chernicoff et al. (2014) proposed that Rhyacian terrane amalgamation would have been preceded by the Siderian extension of a 
Table 3

Lu-Hf composition of zircons from Siempre Amigos hill, Tandilia, Argentina.

\begin{tabular}{|c|c|c|c|c|c|c|c|c|c|c|c|c|}
\hline Sample & $\begin{array}{l}\text { Grain/ } \\
\text { Spot }\end{array}$ & ${ }^{176} \mathrm{Lu} /{ }^{177} \mathrm{Hf}$ & $\begin{array}{l}{ }^{176} \mathrm{Lu} /{ }^{177} \mathrm{Hf} \\
(2 \mathrm{SE})\end{array}$ & ${ }^{176} \mathrm{Hf} /{ }^{177} \mathrm{Hf}$ & $\begin{array}{l}{ }^{176} \mathrm{Hf} /{ }^{177} \mathrm{Hf} \\
(2 \mathrm{SE})\end{array}$ & $\begin{array}{l}\mathrm{Hf} \\
(\mathrm{V})\end{array}$ & $\begin{array}{l}\text { U_Pb Age } \\
\text { (Ma) }\end{array}$ & $\begin{array}{l}{ }^{176} \mathrm{Hf} /{ }^{177} \mathrm{Hf} \\
\text { inSAMPLE }\end{array}$ & $\begin{array}{l}{ }^{176} \mathrm{Hf} /{ }^{177} \mathrm{Hf}_{\mathrm{t}} \\
\text { CHUR }\end{array}$ & $\mathrm{eHf}^{(\mathrm{t})}$ & $\mathrm{T}_{\mathrm{DM}}$ & $\mathrm{T}_{\mathrm{DM}}^{\mathrm{c}}$ \\
\hline 0308 & 2.1 & 0.0003800 & 0.0000370 & 0.281330 & 0.000100 & 4.3 & 2108 & 0.281314746 & 0.281436194 & -4.32 & 2659 & 3244 \\
\hline 0308 & 2.2 & 0.0004220 & 0.0000360 & 0.281330 & 0.000110 & 3.4 & 2143 & 0.281312778 & 0.281413741 & -3.59 & 2665 & 3257 \\
\hline 0308 & 3.1 & 0.0008730 & 0.0000220 & 0.281390 & 0.000170 & 2.7 & 2112 & 0.281354887 & 0.281433584 & -2.80 & 2639 & 3206 \\
\hline 0308 & 4.1 & 0.0007840 & 0.0000530 & 0.281352 & 0.000096 & 3.6 & 2125 & 0.281320275 & 0.281425361 & -3.73 & 2680 & 3253 \\
\hline 0308 & 5.1 & 0.0004080 & 0.0000480 & 0.281434 & 0.000094 & 3.5 & 2179 & 0.28141706 & 0.281389967 & 0.96 & 2524 & 3128 \\
\hline 0308 & 6.1 & 0.0005740 & 0.0000160 & 0.281447 & 0.000080 & 4.2 & 2136 & 0.281423643 & 0.281417789 & 0.21 & 2526 & 3112 \\
\hline 0308 & 7.1 & 0.0003190 & 0.0000430 & 0.281430 & 0.000200 & 4.0 & 2094 & 0.281417281 & 0.281445328 & -1.00 & 2518 & 3102 \\
\hline 0308 & 8.1 & 0.0007630 & 0.0000890 & 0.281520 & 0.000120 & 2.8 & 2063 & 0.281490034 & 0.281465414 & 0.87 & 2448 & 3008 \\
\hline 0308 & 9.1 & 0.0009500 & 0.0001900 & 0.281670 & 0.000250 & 2.7 & 2066 & 0.281632639 & 0.281463588 & 6.01 & 2265 & 2820 \\
\hline 0308 & 33.1 & 0.0003206 & 0.0000061 & 0.281500 & 0.000240 & 2.4 & 2098 & 0.281487194 & 0.281442849 & 1.58 & 2424 & 3010 \\
\hline 0308 & 32.1 & 0.0004841 & 0.0000081 & 0.281390 & 0.000089 & 3.2 & 2096 & 0.281370681 & 0.281444154 & -2.61 & 2591 & 3169 \\
\hline 0308 & 31.1 & 0.0010120 & 0.0000470 & 0.281546 & 0.000083 & 3.1 & 2100 & 0.28150554 & 0.281441675 & 2.27 & 2443 & 3003 \\
\hline 0308 & 10.1 & 0.0006630 & 0.0000630 & 0.281490 & 0.000110 & 3.8 & 2088 & 0.28146365 & 0.281449633 & 0.50 & 2477 & 3047 \\
\hline 0308 & 12.1 & 0.0009690 & 0.0000500 & 0.281460 & 0.000140 & 3.5 & 2063 & 0.281421952 & 0.281465675 & -1.55 & 2554 & 3105 \\
\hline 0308 & 13.1 & 0.0008000 & 0.0001200 & 0.281640 & 0.000130 & 3.9 & 2150 & 0.281607236 & 0.28140891 & 7.05 & 2290 & 2874 \\
\hline 0308 & 13.2 & 0.0007430 & 0.0000380 & 0.281556 & 0.000092 & 3.8 & 2104 & 0.281526234 & 0.281438935 & 3.10 & 2397 & 2970 \\
\hline 0308 & 15.1 & 0.0009910 & 0.0000530 & 0.281530 & 0.000180 & 3.1 & 2771 & 0.28147738 & 0.281000902 & 16.96 & 2480 & 3223 \\
\hline 0308 & 29.1 & 0.0005370 & 0.0000160 & 0.281514 & 0.000085 & 3.5 & 2014 & 0.281493429 & 0.281497866 & -0.16 & 2429 & 2983 \\
\hline 0308 & 16.1 & 0.0004660 & 0.0000260 & 0.281330 & 0.000230 & 2.7 & 2098 & 0.281311387 & 0.28144298 & -4.68 & 2670 & 3248 \\
\hline 0308 & 17.1 & 0.0004370 & 0.0000130 & 0.281450 & 0.000120 & 3.8 & 2102 & 0.281432508 & 0.281440109 & -0.27 & 2505 & 3087 \\
\hline 0308 & 18.1 & 0.0006430 & 0.0000370 & 0.281473 & 0.000082 & 3.0 & 2181 & 0.281446281 & 0.281388791 & 2.04 & 2500 & 3096 \\
\hline 0308 & 20.2 & 0.0004560 & 0.0000180 & 0.281457 & 0.000083 & 3.5 & 2120 & 0.28143859 & 0.281428494 & 0.36 & 2498 & 3084 \\
\hline 0308 & 19.1 & 0.0005420 & 0.0000520 & 0.281461 & 0.000083 & 4.2 & 2103 & 0.281439297 & 0.281439587 & -0.01 & 2502 & 3081 \\
\hline 0308 & 21.2 & 0.0007500 & 0.0000460 & 0.281518 & 0.000087 & 3.3 & 2111 & 0.281487852 & 0.281434367 & 1.90 & 2450 & 3023 \\
\hline 0308 & 23.1 & 0.0003389 & 0.0000063 & 0.281501 & 0.000087 & 3.5 & 2053 & 0.281487756 & 0.281471933 & 0.56 & 2424 & 2997 \\
\hline 0308 & 24.1 & 0.0003670 & 0.0000240 & 0.281500 & 0.000079 & 4.2 & 2121 & 0.281485173 & 0.28142758 & 2.05 & 2429 & 3021 \\
\hline 0308 & 25.1 & 0.0006000 & 0.0000340 & 0.281474 & 0.000091 & 4.4 & 2072 & 0.281450336 & 0.281459807 & -0.34 & 2491 & 3059 \\
\hline 1008 & 2.1 & 0.0009080 & 0.0000640 & 0.281496 & 0.000082 & 3.3 & 2135 & 0.28145907 & 0.281418442 & 1.44 & 2499 & 3073 \\
\hline 1008 & 5.1 & 0.0012990 & 0.0000890 & 0.281410 & 0.000120 & 2.9 & 2104 & 0.281357965 & 0.281439065 & -2.88 & 2664 & 3212 \\
\hline 1008 & 31.1 & 0.0016070 & 0.0000250 & 0.281478 & 0.000093 & 2.9 & 2154 & 0.281412054 & 0.281406167 & 0.21 & 2611 & 3160 \\
\hline 1008 & 6.1 & 0.0016580 & 0.0000920 & 0.281482 & 0.000097 & 2.7 & 2092 & 0.281415971 & 0.281446894 & -1.10 & 2609 & 3140 \\
\hline 1008 & 3.1 & 0.0010920 & 0.0000480 & 0.281370 & 0.000140 & 4.1 & 2099 & 0.281326367 & 0.281442458 & -4.12 & 2693 & 3247 \\
\hline 1008 & 7.1 & 0.0009220 & 0.0000160 & 0.281548 & 0.000075 & 3.3 & 2093 & 0.281511267 & 0.281446372 & 2.31 & 2429 & 2991 \\
\hline 1008 & 8.1 & 0.0014000 & 0.0000770 & 0.281350 & 0.000160 & 3.4 & 2089 & 0.281294322 & 0.28144872 & -5.49 & 2759 & 3297 \\
\hline 1008 & 9.1 & 0.0009380 & 0.0000580 & 0.281430 & 0.000110 & 3.3 & 2142 & 0.281391723 & 0.281413872 & -0.79 & 2593 & 3166 \\
\hline 1008 & 10.1 & 0.0007500 & 0.0000390 & 0.281460 & 0.000130 & 3.5 & 2105 & 0.281429936 & 0.281438152 & -0.29 & 2529 & 3099 \\
\hline 1008 & 11.1 & 0.0004540 & 0.0000220 & 0.281290 & 0.000180 & 3.4 & 2122 & 0.281271657 & 0.28142745 & -5.54 & 2722 & 3307 \\
\hline 1008 & 14.1 & 0.0021400 & 0.0001100 & 0.281390 & 0.000160 & 2.7 & 2092 & 0.28130475 & 0.281446502 & -5.04 & 2799 & 3308 \\
\hline 1008 & 29.1 & 0.0011490 & 0.0000560 & 0.281442 & 0.000095 & 2.8 & 2138 & 0.281395215 & 0.281416875 & -0.77 & 2603 & 3166 \\
\hline 1008 & 15.1 & 0.0013360 & 0.0000850 & 0.281470 & 0.000140 & 4.9 & 2102 & 0.281416525 & 0.281440109 & -0.84 & 2586 & 3133 \\
\hline 1008 & 16.1 & 0.0009800 & 0.0001000 & 0.281460 & 0.000170 & 3.9 & 2072 & 0.281421345 & 0.281459676 & -1.36 & 2556 & 3108 \\
\hline 1008 & 17.1 & 0.0016000 & 0.0000300 & 0.281478 & 0.000098 & 2.6 & 2113 & 0.28141361 & 0.281432801 & -0.68 & 2608 & 3147 \\
\hline 1008 & 21.1 & 0.0013700 & 0.0001500 & 0.281560 & 0.000120 & 4.7 & 2073 & 0.281505951 & 0.281459415 & 1.65 & 2465 & 3004 \\
\hline
\end{tabular}

proto-RPC, followed by the development of narrow oceans, sedimentation over a transitional continental-to-oceanic crust, and arc magmatism in the early Rhyacian. Although Archean ages have not been found in the RPC so far, Hf $\mathrm{T}_{\mathrm{DM}}$ isotopic data presented in the present study, similar to those provided by Cingolani (2011) and Santos et al. (2017), plus scarce inherited Neoarchean zircons, suggest the existence of a veiled Neoarchean crust, perhaps completely recycled during the stages of RPC evolution which followed. Even though it is speculative to interpret a configuration for this pre-Neoarchean stage (ca. $2.6 \mathrm{Ga}$ ), the juvenile nature of SA Neoarchean-inherited zircon, and its scarcity, point to a rock of mafic source. This is consistent with a global context involving the assembly of a hypothetical Archean supercontinent through Superia/Sclavia orogeny, manifested by a $\sim 2.7 \mathrm{Ga}$ peak in detrital zircon spectra, according to Hawkesworth et al. (2017), while the development of new continental crust with or without a supercontinent is also admitted. The hypothetical model proposed, plus the subsequent proposed evolutionary scenario during the early-to-late Rhyacian for the RPC, are also consistent with the two-stage evolution model for late Archean granitoids proposed by Laurent et al. (2014), as described above.

\section{Conclusions}

We present the following concluding remarks:
- New geochemical data for Siempre Amigos Hills, a small but representative portion of the Tandilia basement and RPC southern outcrops, indicate a heterogeneous Paleoproterozoic crust, with mafic (amphibolites, charnockitic?), intermediate (gneiss, diorite) to felsic (granodiorite and granite) compositions, suggesting a differentiation process from an igneous mafic source.

- New U-Pb crystallization ages of $2092 \pm 13$ Ma for granodiorite and $2104 \pm 8$ Ma for intermediate composition gneiss, may arise from 'Camboriu' orogeny and the Trans-Amazonian-Eburnean orogenic cycle.

- This paper is the first to put forward the hypothesis of a sanukitoid precursor for RPC Rhyacian magmatism. As a result of the comparison with granitoids related to the Archean-Paleoproterozoic transitional period only amphibolites show affinities with sanukitoids. Potassic and calc-alkaline arc character, which is indicated by $\mathrm{Nb}-\mathrm{Ta}-\mathrm{Ti}$ typical negative anomalies and REE patterns, provide convincing evidence compatible with normal calcalkaline subduction related magmas. The global tectonic context interpreted in this paper, hypothetically, for Tandilia terrane as a whole, is coherent with the transition between the Archean and Paleoproterozoic eons, characterized by a decrease in the predominance of TTGs, which were progressively replaced by potassium granitoids after $2.5 \mathrm{Ga}$, thus marking evolution towards modern-style tectonics. 


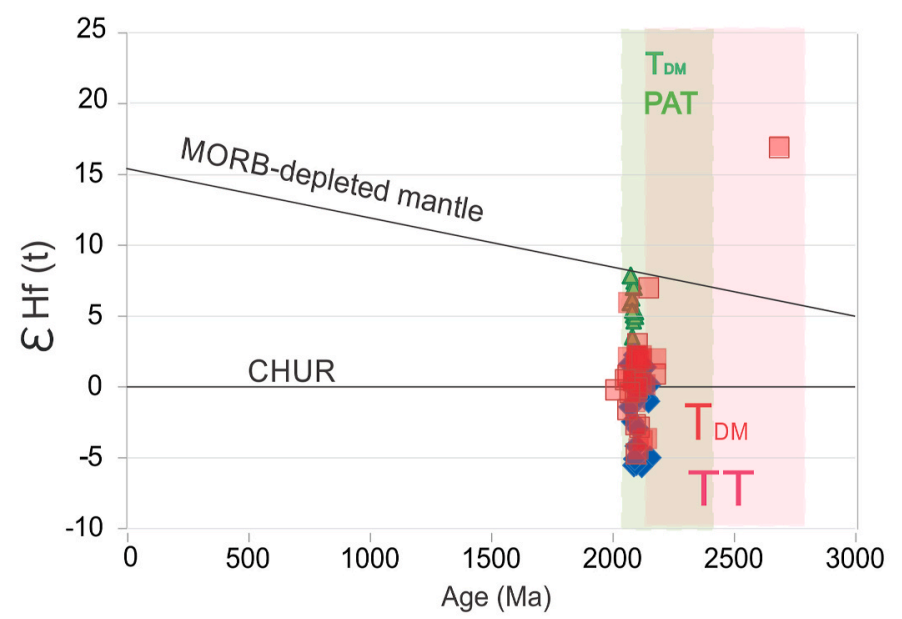

Fig. 15. (a) $\varepsilon H f$ versus U-Pb zircon age for: Siempre Amigos Hills granodiorite (red); Siempre Amigos Hills intermediate composition gneiss (blue); and Cerro Colorado granite (green) from Piedra Alta terrane (after Oriolo et al., 2016). Ranges of model ages ( $\mathrm{T}_{\mathrm{DM}}$ ) are also shown from: Tandilia (TT: pink field); and Piedra Alta terrane (PAT: green field). (b) Plot of ${ }^{176} \mathrm{Hf} /{ }^{177} \mathrm{Hf}$ initial ratios versus ages for dated zircon crystals of granodiorite (red) and intermediate composition gneiss (blue) from Siempre Amigos Hills. The Depleted Mantle model-age (crustal) is $2.8 \mathrm{Ga}$ (Neoarchean). The slope of the broken line uses the ${ }^{176} \mathrm{Lu} /{ }^{177} \mathrm{Hf}$ ratio of 0.0113 for average crust (Taylor and McLennan, 1985; Wedepohl, 1995).

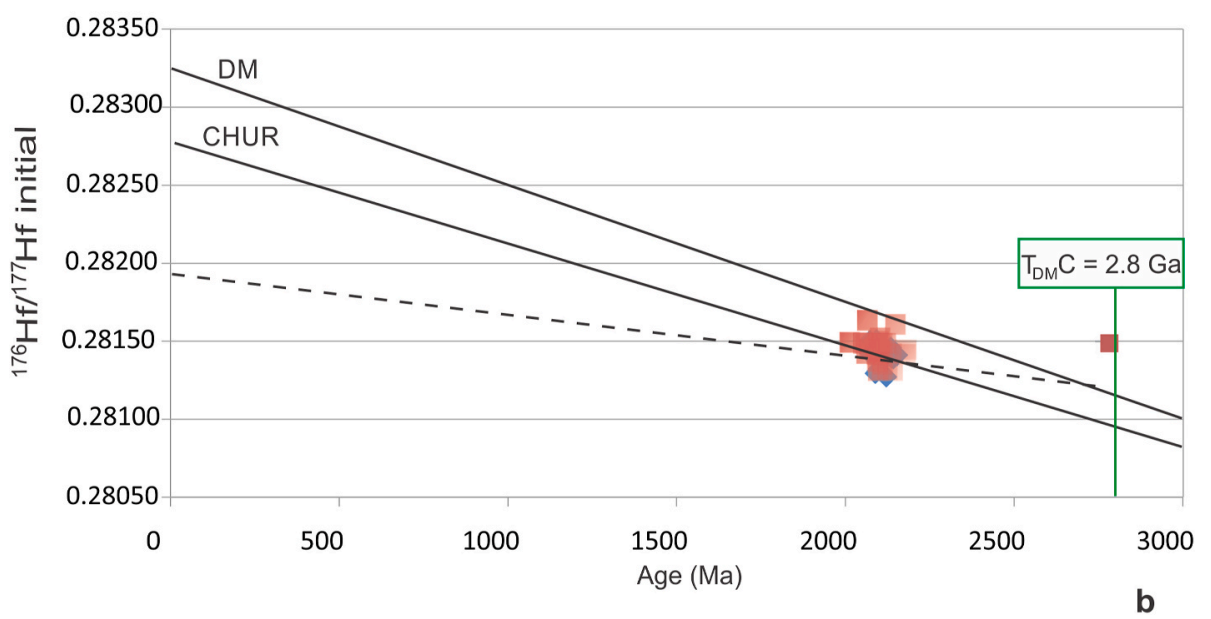

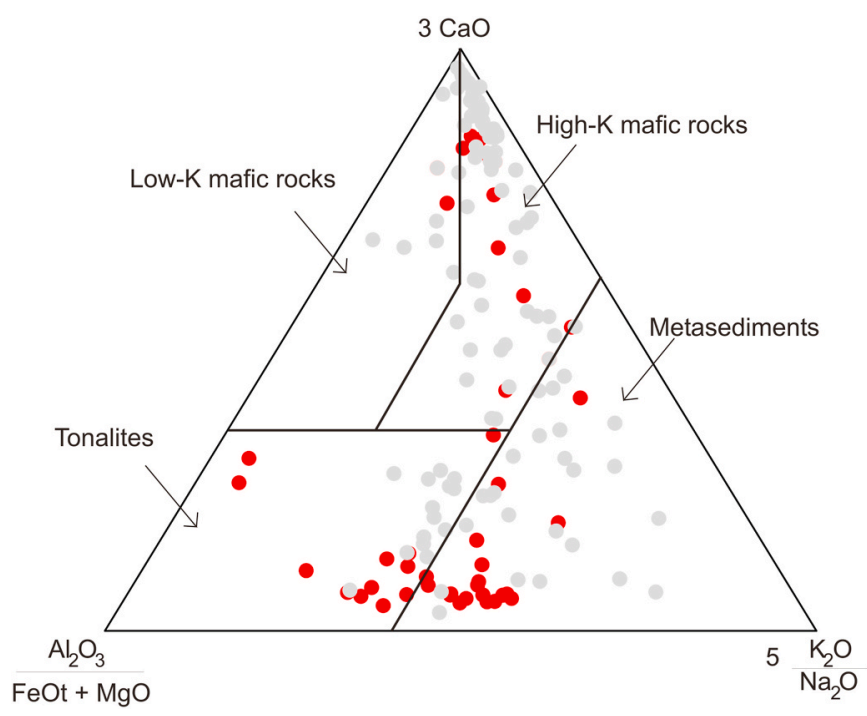

Fig. 16. Triangular plot of $\mathrm{Al}_{2} \mathrm{O}_{3} /(\mathrm{FeOt}+\mathrm{MgO})-3 \mathrm{CaO}-5\left(\mathrm{~K}_{2} \mathrm{O} / \mathrm{Na}_{2} \mathrm{O}\right)$, with fields after Laurent et al. (2014). Red symbols: Siempre Amigos Hills samples. Grey symbols: Tandilia geochemical background.

- A sustainable petrogenetic model is put forward to explain the presence of geochemical and isotopic evidence regarding sanukitoid magmatism which may have occurred about $2.7 \mathrm{Ga}$ or after, and appears to constitute a magmatic series precursor to the development of the Rhyacian magmatic arc in the Tandilia Terrane. Subduction with slab breakoff provides an explanation for the heat required to melt part of the slab, heavily contaminated by the assimilation of mantle peridotite, and create a geochemical sanukitoid signature in SA amphibolites. This region thus presents magmatic arc lowdeformed calc-alkaline granitoids intruding into host rocks of medium-to-high metamorphic grade, except for the localized lowtemperature shear zones that affected both. These metamorphic conditions probably derived from Palaeoproterozoic subduction. Subsequently, syn-to- late-metamorphic intrusion of huge volumes of Rhyacian calc-alkaline magmatism occurred.

- Based on a new Lu-Hf isotopic signature analyzed in SA granodiorite and gneiss, it is assumed that these rocks are cogenetic, with a $2.7 \mathrm{Ga}$ $\mathrm{T}_{\mathrm{DM}}$ age. This magmatic event is predominantly juvenile, with less participation from crustal recycling indicated by both positive and slightly negative $\varepsilon H f$ values. Inheritance is recorded in a single core of Neoarchean zircon from a granodiorite sample (Analysis $15.1=$ $2771.1 \pm 15 \mathrm{Ma}$ ), which shows a degree of similarity with the calculated $\mathrm{HfT}_{\mathrm{DM}}$ model age and the scarce previous Lu-Hf and Sm-Nd information regarding Tandilia terrane.

- This model is compatible with RPC configuration as a collage of parautocthonous terranes. So the existence of other terranes and suture zones cannot be discarded and could provide a direction for future research. Our interpretations are also consistent with the context of Trans-Amazonian-Eburnean orogeny, such as the amalgamation of different microcontinents, including the RPC, during the Paleoproterozoic into the Atlantica configuration. 


\section{Declaration of competing interest}

The authors declare that they have no known competing financial interests or personal relationships that could have appeared to influence the work reported in this paper.

\section{Acknowledgements}

This paper was funded by the following grants: PIP $\mathrm{N}^{\circ}$ 11220150100269 CO and PICT 2012-0644, for which we are grateful to the CONICET and the ANPCyT. The authors are also indebted to the UNS- PGI project (Cod. 24/H137) for the partial funding of field trips. Melisa Angeletti would like to thank the Universidad Nacional del Sur and the Asociación Universitaria Iberoamericana de Postgrado for providing funds for travel to Spain to work with her co-authors. This paper also received funding from the European Union Horizon 2020 research and innovation programme under Marie Skłodowska-Curie grant agreement no. 778045. Martim Chichorro is grateful for the funding provided by the GeoBioTec (UIDB/04035/2020). The authors greatly acknowledged Dr. Sebastian Oriolo for the editorial work and Dr. Mathias Hueck and anonymous reviewer for their constructive suggestions and comments.

\section{References}

Abre, P., Bossi, J., Cingolani, C., Gaucher, C., Piñeiro, D., Blanco, G., 2014. El TerrenoTandilia en Uruguay y Argentina. In: Bossi, J., Gaucher, C. (Eds.), Geología del Uruguay. Tomo 1: Predevonico. Polo, Montevideo, pp. 89-119.

Almeida, F.F.M., Amaral, G., Cordani, U.G., Kawashita, K., 1973. The Precambrian evolution of the South American cratonic margin, south of the Amazon River. In: Nairn, A.E., Stehli, F.G. (Eds.), The Ocean Basins and Margins I. Plenum Publishing, New York, pp. 411-446.

Angeletti, M., Chichorro, M., Frisicale, M.C., Castro, A., Dimieri, L.V., 2014. Nuevas edades radiométricas U-Pb SHRIMP en Tandilia, cerro Siempre Amigos, Sierras de Azul, Buenos Aires, Argentina. $19^{\circ}$ Congreso Geológico Argentino. Sesión Temática Petrología Ígnea y Metamórfica T8-01, p. 2 (Córdoba).

Angeletti, M., Martínez, J.C., Frisicale, M.C., Dimieri, L.V., 2016a. Aplicación del geotermómetro GRT-BT y del geotermobarómetro GRT-PX-BT en la granulita del cerro Siempre Amigos, Sierras de Azul, Tandilia. Acta Geol. Lilloana 14-18, 28, N Extra 1-1 (Ejemplar dedicado a: XII Congreso de Mineralogía y Metalogenia).

Angeletti, M., Frisicale, M.C., Dimieri, L.V., 2016b. Petrografía, caracterización geoquímica y análisis microestructural del granito del cerro Siempre Amigos, sierras de Azul, Tandilia. Rev. Asoc. Geol. Argent. 73 (2), 211-224.

Barbosa, J.S.F., Sabaté, P., 2004. Archean and paleoproterozoic crust of the São Francisco craton, bahia, Brazil: geodynamic features. Precambrian Res. 133, 1-27.

Bickle, M.J., 1986. Implications of melting for stabilisation of the lithosphere and heat loss in the Archaean. Earth Planet Sci. Lett. 80 (3-4), 314-324.

Bossi, J., Cingolani, C., 2009. Extension and general evolution of the Río de la Plata Craton. In: Gaucher, C., Sial, A.N., Halverson, G.P., Frimmel, H.E. (Eds.), Neoproterozoic-Cambrian Tectonics, Global Change and Evolution: a Focus on Southwestern Gondwana, Developments in Precambrian Geology, vol. 16, pp. 73-85.

Bouvier, A., Vervoort, J.D., Patchetta, P.J., 2008. The Lu-Hf and Sm-Nd isotopic composition of CHUR: constraints from unequilibrated chondrites and implications for the bulk composition of terrestrial planets. Earth Planet Sci. Lett. 273 (1-2), $48-57$.

Brito Neves, B.B., 2011. The Paleoproterozoic in the South-American continent: diversity in the geologic time. J. S. Am. Earth Sci. 32 (4), 270-286.

Bruno, H., Elizeu, V., Heilbron, M., de Morisson Valeriano, C., Strachan, R., Fowler, M. Bersan, S., Moreira, H., Dussin, I., do Eirado Silva, L.G., Tupinamba, M., Almeida, J., Neto, C., Storey, C., 2020. Neoarchean and Rhyacian TTG-Sanukitoid suites in the southern Sao Francisco Paleocontinent, Brazil: evidence for diachronous change towards modern tectonics. Geoscience Frontiers 11 (5), 1763-1787.

Chappell, B.W., White, A.J.R., 1974. Two contrasting granite types. Pac. Geol. 8, 173-174.

Chauvel, C., Blinchert-Toft, J.E., 2001. A hafnium isotope and trace element perspective on melting of the depleted mantle. Earth Planet Sci. Lett. 190 (3-4), 137-151.

Chernicoff, J.C., Zappettini, E.O., Peroni, J., 2014. The Rhyacian El Cortijo suture zone: aeromagnetic signature and insights for the geodynamic evolution of the southwestern Rio de la Plata craton, Argentina. Geoscience Frontiers 5, 43-52.

Chernicoff, C.J., Zappettini, E.O., Santos, J.O., Pesce, A., McNaughton, N.J., 2015. Zircon and Titanite U-Pb SHRIMP dating of unexposed basement units of the Buenos Aires region, southeastern Río de La Plata Craton, Argentina. Int. Geol. Rev. 58 (5), 643-652.

Chu, N.C., Taylor, R.N., Chavagnac, V., Nesbitt, R.W., Boella, R.M., Milton, J.A., German, C.R., Bayon, G., Burton, K., 2002. Hf isotope ratio analysis using multicollector inductively coupled plasma mass spectrometry: an evaluation of isobaric interference corrections. J. Anal. At. Spectrom. 17, 1567-1574.
Cingolani, C.A., Hartmann, L.A., Santos, J.O.S., McNaughton, N.J., 2002. U-Pb SHRIMP dating of zircons from the Buenos Aires complex of the Tandilia belt, Río de La Plata craton, Argentina. $15^{\circ}$ Congreso Geológico Argentino 149-154. El Calafate, Santa Cruz 1.

Cingolani, C.A., Santos, J.O.S., Griffin, W., 2010. New insights of the Paleoproterozoic basement of Tandilia belt, Río de la Plata craton, Argentina: first Hf isotope studies on zircon crystals. In: Symposium GEOSUR, Extended Abstract, Mar del Plata, Argentina. Bollettino di Geofisica teorica ed applicata. An International Journal of Earth Sciences, vol. 51, pp. 21-24.

Cingolani, A., 2011. The Tandilia System of Argentina as a southern extension of the Río de la Plata craton: an overview. Int. J. Earth Sci. 100, 221-242.

Condie, K.C., 2005. TTGs and adakites: are they both slab melts? Lithos 80 (1-4), 33-44.

Coutinho, J., Kräutner, H., Sassi, F., Schmid, R., Sen, S., 2007. Recommendations by the IUGS Subcommission on the Systematics of Metamorphic Rocks. Web version 01.02.07.

Dalla Salda, L.H., Franzese, J.R., Posadas, V.G., 1992. The 1800 Ma mylonite-anatectic granitoid association in Tandilia, Argentina. International Basement Tectonics 7, 161-174. Association Publication.

Dalla Salda, L., Spalletti, L., Poiré, D., de Barrio, R., Echeveste, H., Benialgo, A., 2006 Tandilia. INSUGEO. Serie Correlación Geológica 21, 17-46.

Delor, C., Lahondère, D., Egal, E., Lafon, J.-M., Cocherie, A., Guerrot, C., Rossi, P., Truffert, C., Théveniaut, H., Phillips, D., de Avelar, V.G., 2003. Transamazonian Crustal Growth and Reworking as Revealed by the 1: 500,000-Scale Geological Map of French Guiana, second ed. Géologie de la France. 2-3-4, 5-57.

Dhuime, B., Hawkesworth, C.J., Cawood, P.A., Storey, C.D., 2012. A change in the geodynamics of continental growth 3 billion years ago. Science 335, 1334-1336.

Di Paola, E.C., Espiasse, García, 1986. Génesis del yacimiento de caolín Cerro Segundo, sierra de Bachicha, partido de Balcarce, Provincia de Buenos Aires. Argentina. Revista de la Asociación Argentina de Mineralogía, Petrología y Sedimentología 17 (1-4), 47-54.

Di Paola, E.C., 1995. Caracterización petrográfica y química de la alteración caolinítica del yacimiento María Eugenia, provincia de Buenos Aires, Argentina. In: Actas IV Jornadas Geológicas y Geofísicas Bonaerenses, Junín, vol. 1.

Drummond, M.S., Defant, M.J., 1990. A Model for trondhjemite-tonalite-dacite genesis and crystal growth via slab melting: Archean to modern comparisons. J. Geophys. Res. 95 (13), 21,503-521,521.

Foley, S.F., Barth, M.G., Jenner, G.A., 2000. Rutile/melt partition coefficient for trace elements and an assessment of the influence of rutile on the trace element characteristics of subduction zone magmas. Geochimica et Cosmochimica Acta. Acta 64, 933-938.

Fowler, M., Rollinson, H., 2012. Phanerozoic sanukitoids from caledonian scotland: implications for archean subduction. Geology 40, 1079-1082.

Frisicale, M.C., Dimieri, L.V., Dristas, J.A., 2001. Cinemática de las milonitas del basamento en Boca de la Sierra, Sierras de Azul, Buenos Aires. Rev. Asoc. Geol. Argent. 56 (3), 319-330.

Frisicale, M.C., Martinez, J.C., Dimieri, L.V., Dristas, J.A., 2005. Microstructural analysis and P-T conditions of the Azul megashear zone, Tandilia, Buenos Aires province, Argentina. J. S. Am. Earth Sci. 19, 433-444.

Frisicale, M.C., Dimieri, L.V., Dristas, J.A., Araujo, V., Fortunatti, N., 2012. Microstructural and geochemical analysis of Paleoproterozoic pseudotachylytes in Río de la Plata craton, Tandilia belt, Argentina. Geol. Acta 2 (10), 85-101.

Frost, C.D., Frost, B.R., Chamberlain, K.R., Hulsebosch, T.P., 1998. The Late Archaean history of the Wyoming province as recorded by granitic magmatism in the Wind River Range, Wyoming. Precambrian Res. 89, 145-173.

Frost, B.R., Arculus, R.J., Barnes, C.G., Collins, W.J., Ellis, D.J., Frost, C.D., 2001 A geochemical classification of granitic rocks. J. Petrol. 42, 2033-2048.

Frost, B.R., Frost, C.D., 2008a. A geochemical classification for feldspathic igneous rocks. J. Petrol. 49 (11), 1955-1969.

Frost, B.R., Frost, C.D., 2008b. On charnockites. Gondwana Res. 13, 30-44.

Gianotti, V., 2009. Caracterización geológico-estructural de las zonas de cizalla de Colonia. PhD thesis. Facultad de Ciencias, Universidad de la República, Montevideo.

González Bonorino, F., Zardini, R., Figueroa, M., Limousin, T., 1956. Estudio geológico de las Sierras de Olavarría y Azul (Prov. de Buenos Aires). LEMIT, Serie 2 (63), 1-22.

Grove, T.L., Elkins-Tanton, L.T., Parman, W.S., Chatterjee, N., Mutener, O., Gaetani, G. A., 2003. Fractional crystallization and mantle-melting controls on calc-alkaline differentiation trends. Contrib. Mineral. Petrol. 145, 515-533.

Halla, J., can Hunen, J., Heilmo, E., Hottla, P., 2009. Geochemical and numerical constraints on Neoarchean plate tectonics. Precambrian Res. 175, 155-162.

Hartmann, L.A., 2002. The mesoproterozoic supercontinent Atlantica in the Brazilian shield. Review of geological and U-Pb zircon and Sm-Nd isotopic evidence. Gondwana Res. 5 (I), 157-163.

Hartmann, L.A., Santos, J.O.S., Cingolani, C.A., McNaughton, N.J., 2002. Two paleoproterozoic orogenies in the evolution of the Tandilia belt, Buenos Aires, as evidenced by zircon U-Pb SHRIMP geochronology. Int. Geol. Rev. 44, 528-543.

Hawkesworth, C.J., Kemp, A.I.S., 2006. Evolution of the continental crust. Nature 443, 811-817.

Hawkesworth, C.J., Cawood, P.A., Dhuime, B., Kemp, T.I.S., 2017. Earth's continental lithosphere through time. Annu. Rev. Earth Planet Sci. 45, 169-198.

Hoffman, P.F., 1997. Tectonic genealogy of north America. In earth structure. In: Van der Pluijm, B.A., Marshak, S. (Eds.), An Introduction to Structural Geology and Tectonics. McGraw-Hill, New York, pp. 459-464.

Hoskin, P.W.O., Schaltegger, U., 2003. The composition of zircon and igneous and metamorphic petrogenesis. In: Hanchar, J.M., Hoskin, P.W.O. (Eds.), Zircon. Review in Mineralogy and Geochemistry, vol. 53, pp. 27-62. https://doi.org/10.2113/ 0530027. 
Iacumin, M., Piccirillo, E.M., Girardi, V.A.V., Teixeira, W., Bellieni, G., Echeveste, H., Fernandez, R., Pinese, J.P.P., Ribot, A., 2001. Early proterozoic calc-alkaline and middle proterozoic tholeiitic dyke swarms from Central-Eastern Argentina: petrology, geochemistry, Sr- Nd Isotopes and Tectonic implications. J. Petrol. 42 (11), 2109-2143.

Jorgensen, L., Frisicale, M.C., Dimieri, L.V., 2008. Microestructuras de deformación en rocas granulíticas, cerro La Crespa, Tandilia, Prov, Bs as. Rev. Asoc. Geol. Argent. 63 (2), 233-243.

Klemme, S., Gunther, D., Hametner, K., Prowatke, S., Zack, T., 2006. The partitioning of trace elements between ilmenite, ulvospinel, armalcolite and silicate melts with implications for the early differentiation of the moon. Chem. Geol. 234, 251-263.

Larrea, M.L., Castro, S.M., Bjerg, E.A., 2014. A software solution for point counting. Petrographic thin section analysis as a case study. Arabian Journal of Geosciences 7 (8), 2981-2989.

Laurent, O., Martin, F., Moyen, J.F., Doucelance, R., 2014. The diversity and evolution of late-Archean granitoids: evidence for the onset of "modern-style" plate tectonics between 2.0 and 2.5 Ga. Lithos 205, 208-235.

Le Bas, M., Le Maitre, R., Streckeisen, A., Zanettin, B., 1986. A chemical classification of volcanic rocks based on the total alkali-silica diagram. J. Petrol. 27 (3), 745-750.

Ledru, P., Johan, V., Millesi, J.P., Tegyey, M., 1994. Markers of the last stages of the Paleoproterozoic collision: evidence for a $2.0 \mathrm{Ga}$ continent involving circum-Atlantic provinces. Precambrian Res. 69, 169-191.

Lobach-Zhuchenko, S.B., Rollinson, H., Chekulaev, V.P., Savatenkov, V.M., Kovalenko, A.V., Martin, H., Guseva, N.S., Arestova, N.A., 2008. Petrology of a late archaean, highly potassic, sanukitoid pluton from the baltic shield: insights into late archaean mantle metasomatism. J. Petrol. 49, 393-420.

Ludwig, K.R., 2003. Isoplot (3.41d), a Geochronological Toolkit for Excel. Berkeley Geochronology Center Special Publication. No. 4.

Marchese, H., Di Paola, E., 1975. Reinterpretación estratigráfica de la perforación de Punta Mogotes I, Provincia de Buenos Aires. Rev. Asoc. Geol. Argent. 30 (1), 44-52.

Martin, H., 1986. Effect of steeper Archean geothermal gradient on geochemistry of subduction-zone magmas. Geology 14, 753-756.

Martin, H., 1999. The adakitic magmas: modern analogues of Archaean granitoids. Lithos 46, 411-429.

Martin, H., Smithies, R., Rapp, R.P., Moyen, J.F., Champion, D., 2005. An overview of adakite, TTG, and sanukitoid: relationships and some implications for crustal evolution. Lithos 79 (1-2), 1-24.

Martin, H., Moyen, J.F., Rapp, R., 2009. The sanukitoid series: magmatism at the Archaean-Proterozoic transition. Trans. Earth Sci. 100, 15-33.

Martin, H., Moyen, J.-F., 2012. Forty years of TTG research. Lithos 148, 312-336.

Martínez, J.C., Dristas, J.A., 2007. Paleoactividad hidrotermal en la discordancia entre el complejo Buenos Aires y la formación La Tinta en el área de Barker, Tandilia. Rev. Asoc. Geol. Argent. 62 (3), 375-386.

Massonne, H.J., Dristas, J.A., Martínez, J.C., 2012. Metamorphic evolution of the Río de la Plata craton in the Cinco Cerros área, Buenos Aires province, Argentina. J. S. Am. Earth Sci. 38, 57-70.

Miller, C.F., Stoddard, E.F., 1981. The role of manganese in the paragenesis of magmatic garnet: an Example from the Old Woman-Piute Range, California. J. Geol. 89, $233-246$.

Miller, C.F., McDowell, S.M., Mapes, R.W., 2003. Hot and cold granites? Implications of zircon saturation temperatures and preservation of inheritance. Geology 31, 529-532.

Montefalco de Lira Santos, L.C., Dantas, E.L., Cawood, P.A., Santos, E.J., 2017. Neoarchean crustal growth and Paleoproterozoic reworking in the Borborema Province, NE Brazil: insights from geochemical and isotopic data of TTG and metagranitic rocks of the Alto Moxotó Terrane. J. S. Am. Earth Sci. 79, 342-363.

Moreira, H., Storey, C., Fowler, M., Seixas, L., Dunlop, J., 2020. Petrogenetic processes at the tipping point of plate tectonics: Hf-O isotope ternary modelling of Earth's last TTG to sanukitoid transition. Earth Planet Sci. Lett. 551, 116558.

Oriolo, S., Oyhantçabal, P., Basei, M.A., Wemmer, K., Siegesmund, S., 2016. The Nico Perez terrane (Uruguay): from archean crustal growth and connections with the Congo craton to late neoproterozoic accretion to the Río de la Plata craton. Precambrian Res. 280, 147-160.

Oyhantçabal, P., Wemmer, K., Siegesmund, S., 2006. K/Ar geochronology of the Mosquitos shear zone (Piedra alta terrane-río de la Plata craton-Uruguay). In: 5th South American symposium on isotope geology, Punta del Este, brief paper, p. 149.

Oyhantçabal, P., Siegesmund, S., Wemmer, K., 2011. The Río de la Plata Craton: a review of units, boundaries, ages and isotopic signature. Int. J. Earth Sci. 100, 201-220.

Oyhantçabal, P., Cingolani, C., Wemmer, K., Siegesmund, S., 2018. The Rio de la Plata craton of Argentina and Uruguay. In: Siegesmund, S., Stipp Basei, M.A., Oyhantçabal, P., Oriolo, S. (Eds.), Geology of Southwest Gondwana. Springer Nature.

Pacheco Neves, S., 2003. Proterozoic history of the Borborema province (NE Brazil): correlations with neighboring cratons and Pan-African belts and implications for the evolution of western Gondwana. Tectonics 22 (4), 1031, 5,1-5,14.

Pamoukaghlian, K., Gaucher, C., Frei, R., Poiré, D.G., Chemale, F., Frei, D., Will, T.M., 2017. U-Pb age constraints for the La tuna granite and montevideo formation (paleoproterozoic, Uruguay): unravelling the structure of the Río de la Plata craton. J. S. Am. Earth Sci. 79, 443-458.

Pankhurst, R.J., Ramos, A., Linares, E., 2003. Antiquity of the Río de la Plata craton in Tandilia, southern Buenos Aires province, Argentina. J. S. Am. Earth Sci. 16, 5-13. Passchier, C., Trouw, R., 2005. Microtectonics. Springer Verlag, Berlin, p. 366.

Paton, C., Hellstrom, J., Paul, B., Woodhead, J., Hergt, J., 2011. Iolite: freeware for the visualisation and processing of mass spectrometric data. J. Anal. At. Spectrom. 26 (12), 2508-2518.
Pearce, J.A., Harris, B.W., Tindle, A.G., 1984. Trace element discrimination diagrams for the tectonic interpretation of granitic rocks. J. Petrol. 25, 956-983.

Pearce, J.A., 1996. A user's guide to basalt discrimination diagrams. In: Wyman, D.A. (Ed.), Trace Element Geochemistry of Volcanic Rocks: Applications for Massive Sulphide Exploration Geological Association of Canada, Short Course Notes, vol. 12, pp. 79-113.

Peccerillo, A., Taylor, S.R., 1976. Geochemistry of eocene calc-alkaline volcanic rocks from the Kastamonu area, Northern Turkey. Contrib. Mineral. Petrol. 58, 63-81.

Ramos, V.A., 1999. Rasgos estructurales del territorio Argentino. 1. Evolución tectónica del territorio argentino. In: Caminos, R. (Ed.), Geología Argentina, Anales SEGEMAR 29, Buenos Aires, vol. 24, pp. 715-784.

Rapalini, A.E., Sánchez Bettucci, L., Badgen, E., Vásquez, C.A., 2015. Paleomagnetic study on mid-Paleoproterozoic rocks from the Rio de la Plata craton: implications for Atlantica. Gondwana Res. 27 (4), 1534-1549.

Rapela, C.W., Pankhurst, R.J., Casquet, C., Fanning, C.M., Baldo, E.G., GonzálezCasado, J.M., Galindo, C., Dahlquist, J., 2007. The Río de la Plata craton and the assembly of SW Gondwana. Earth Sci. Rev. 83, 49-82.

Rapela, C.W., Fanning, M., Casquet, C., Pankhurst, R.J., Spalletti, L., Poiré, D., Baldo, E. G., 2011. The Río de la Plata Craton and the adjoining Pan-African/Brasiliano terranes: their origins and incorporation into south-west Gondwana. Gondwana Res. 20, 673-690.

Ribot, A., Bossi, J., Cingolani, C., Piñeyro, D., 2005. Caracterización petrográfica y cinemática de la faja milonítica Colonia y Arroyo Pavón en el sur del Terreno Piedra alta. Uruguay: zona de cizalla principal en basamento precámbrico. In: Actas XVI, Congreso Geológico Argentino. La Plata.

Robertson, S., 1999. BGS Rock classification scheme. In: Classification of Metamorphic Rocks, vol. 2. British Geological Survey Research Report, London, 92-02.24.

Rogers, J.J.W., 1996. A history of continents in the past three billion years. J. Geol. 104, 91-107.

Rogers, J.J.W., Santosh, M., 2002. Configuration of Columbia, a mesoproterozoic supercontinent. Gondwana Res. 5, 5-22.

Rollinson, H.R., 1993. Using Geochemical Data: Evaluation, Presentation, Interpretation. Longman Geochemistry Series.

Sánchez Bettucci, L., Peel, E., Oyhantçabal, P., 2010. Precambrian geotectonic units of the Rio de la Plata craton. Int. Geol. Rev. 52, 32-50.

Santos, J.O.S., Chernicoff, C.J., Zappettini, E.O., McNaughton, N.J., Greau, Y., 2017. U$\mathrm{Pb}$ geochronology of martín garcía, sola, and dos hermanas islands (Argentina and Uruguay): unveiling rhyacian, statherian, ectasian, and stenian of a forgotten area of the Río de la Plata craton. J. S. Am. Earth Sci. 80, 207-228.

Semprich, J., Moreno, J.A., Oliveira, E.P., 2015. Phase equilibria and trace element modeling of Archean sanukitoid melts. Precambrian Res. 269, 122-138.

Shand, S.J., 1947. The Eruptive Rocks, third ed. John Wiley, New York, p. 444.

Shirey, S.B., Hanson, G.N., 1984. Mantle-derived Archaean monzodiorites and trachyandesites. Nature 310, 222-224.

Söderlund, U., Patchett, P.J., Vervoort, J.D., Isachsen, C.E., 2004. The ${ }^{176} \mathrm{Lu}$ decay constant determined by Lu-Hf and U-Pb isotope systematics of Precambrian mafic intrusions. Earth Planet Sci. Lett. 219, 311-324.

Stevenson, R., Henry, P., Gariépy, C., 1999. Assimilation-fractional crystallization origin of archean sanukitoid suites: western superior province, Canada. Precambrian Res. 96, 83-99.

Sun, S.S., McDonough, W.F., 1989. Chemical and isotopic systematics of oceanic basalts: implications for mantle composition and processes. Geological Society, London, Special Publications 42 (1), 313-345.

Tassinari, C.C.G., Macambira, M.J.B., 1999. Geochronological provinces of the amazonian craton. Episodes 22, 174-182.

Taylor, S.R., McLennan, M., 1985. The Continental Crust: its Composition and Evolution. Blackwell, Oxford, p. 312.

Taylor, S.R., McLennan, S.M., 1995. The geochemical evolution of the continental crust. Rev. Geophys. 33, 241-265.

Teixeira, W., Pinese, J.P.P., Iacumin, M., Girardi, V.A.V., Piccirillo, E.M., Echeveste, H., Ribot, A., Fernandez, R., Renne, P., Heaman, L.M., 2002. Calc-alkaline and tholeiitic dyke swarms of Tandilia, Rio de la Plata craton, Argentina: U-Pb, Sm-Nd and Rb-Sr 40Ar-39Ar data provide new clues for intraplate rifting shortly after Transamazonian orogeny. Precambrian Res. 119, 329-353.

Teruggi, M.E., Leguizamón, M., Ramos, V., 1988. Metamorfitas de bajo grado con afinidades oceánicas en el basamento de Tandil: implicaciones geotectónicas, provincia de Buenos Aires. Rev. Asoc. Geol. Argent. 43 (3), 366-374.

Watson, E.B., Harrison, T.M., 1983. Zircon saturation revisited: temperature and composition effects in a variety of crustal magma types. Earth Planet Sci. Lett. 64, 295-304.

Wedepohl, K.H., 1995. The compositions of the continental crust. Geochem. Cosmochim. Acta 59, 1217-1232.

Whitney, D.L., Evans, B.W., 2010. Abbreviations for names of rock-forming minerals. Am. Mineral. 95, 185-187.

Williams, I.S., 1998. U-Th-Pb geochronology by ion microprobe. In: McKibben, M.A., Shanks, W.C., Ridley, W.I. (Eds.), Applications of Microanalytical Techniques to Understanding Mineralizing Processes, Reviews in Economic Geology,, vol. 7, pp. 1-35.

Williams, I.S., Hergt, J.M., 2000. U-Pb dating of Tasmanian dolerites: a cautionary tale of SHRIMP analysis of high-U zircon. In: Woodhead, J.D., Hergt, J.M., Noble, W.P. (Eds.), Beyond 2000: New Frontiers in Isotope Geoscience, Lorne, Abstracts and Proceedings, pp. 185-188. 
Windley, B.F., 1995. The Evolving Continents, third ed. ed. John Wiley \& Sons Ltd., Chichester.

Zen, E., 1988. Phase relations of peraluminous granitic rocks and their petrogenetic implications. Annu. Rev. Earth Planet Sci. 16, 21-52.
Zhao, G., Cawood, P.A., Wilde, S.A., Sun, M., 2002. Review of global 2.1-1.8 Ga orogens: implications for a pre-Rodinia supercontinent. Earth Sci. Rev. 59, 125-162. 